\title{
Eyewitness identification for multiple perpetrator crimes
}

Citation for published version (APA):

Tupper, N. (2018). Eyewitness identification for multiple perpetrator crimes: Examining recognition memory and decisions for multiple faces. [Doctoral Thesis, Maastricht University]. Maastricht University. https://doi.org/10.26481/dis.20180620nt

Document status and date:

Published: 01/01/2018

DOI:

10.26481/dis.20180620nt

Document Version:

Publisher's PDF, also known as Version of record

\section{Please check the document version of this publication:}

- A submitted manuscript is the version of the article upon submission and before peer-review. There can be important differences between the submitted version and the official published version of record.

People interested in the research are advised to contact the author for the final version of the publication, or visit the DOI to the publisher's website.

- The final author version and the galley proof are versions of the publication after peer review.

- The final published version features the final layout of the paper including the volume, issue and page numbers.

Link to publication

\footnotetext{
General rights rights.

- You may freely distribute the URL identifying the publication in the public portal. please follow below link for the End User Agreement:

www.umlib.nl/taverne-license

Take down policy

If you believe that this document breaches copyright please contact us at:

repository@maastrichtuniversity.nl

providing details and we will investigate your claim.
}

Copyright and moral rights for the publications made accessible in the public portal are retained by the authors and/or other copyright owners and it is a condition of accessing publications that users recognise and abide by the legal requirements associated with these

- Users may download and print one copy of any publication from the public portal for the purpose of private study or research.

- You may not further distribute the material or use it for any profit-making activity or commercial gain

If the publication is distributed under the terms of Article $25 \mathrm{fa}$ of the Dutch Copyright Act, indicated by the "Taverne" license above, 


\section{Eyewitness identification for multiple perpetrator crimes:}

Examining recognition memory and decisions for multiple faces

Nina Tupper 
(C) Nina Tupper, Maastricht 2018

ISBN: 978-94-6233-991-0 


\title{
Maastricht University
}

\section{Eyewitness identification for multiple perpetrator crimes:}

\section{Examining recognition memory and decisions for multiple faces}

\author{
Dissertation
}

To obtain the degree of Doctor of Philosophy from The University of Portsmouth and the degree of Doctor at Maastricht University, on the authority of Rector Magnificus Prof.dr. Rianne M. Letschert, according to the decision of the Board of Deans,

to be defended in public on Wednesday $20^{\text {th }}$ of June 2018 at 10.00 hours in Maastricht

by

Nina Tupper

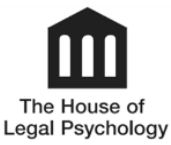




\section{Supervisors:}

Prof.dr. H.L.G.J. Merckelbach

Prof.dr. L. Hope, University of Portsmouth

\section{Co-supervisors:}

Dr. M. Sauerland

Dr. J.D. Sauer, University of Tasmania

\section{Doctoral Thesis Committee}

Prof.dr. M. Jelicic (chair)

Dr. M. Bindemann, University of Kent

Dr. R. Fitzgerald, University of Portsmouth

Prof.dr. E. Rassin, Erasmus University Rotterdam

Prof.dr. C. de Ruiter

The research presented in this dissertation was conducted as a Doctor of Philosophy Program with the House of Legal Psychology in collaboration between the University of Portsmouth and Maastricht University. 




\section{TABLE OF CONTENTS}

Chapter 1: General Introduction 9

$\begin{array}{ll}\text { Chapter 2: } & \text { International police survey }\end{array}$

Eyewitness identification for multiple perpetrator crimes: A survey of police in Sweden, Belgium, and the Netherlands

Chapter 3: Experiments 1, 2 and 3

Showup identification decisions for multiple perpetrator crimes:

Testing for sequential dependencies

Chapter 4: $\quad$ Experiments 4 and 5

Face value: Testing the utility of contextual face cues for face recognition

Chapter 5: General discussion

References

Summary

Valorization

Acknowledgments

Curriculum Vitae 
CHAPTER 1 
CHAPTER 1

\section{General Introduction}




\section{A CASE OF IDENTITY: MISIDENTIFICATION AND MULTIPLE PERPETRATOR CRIMES}

In July of 1981, three men broke into a Florida home, threatened the five victims with a shotgun, and robbed the residence. Three of the victims were tied up and left in the house. Two victims, 12 -year-old Isabelle ${ }^{1}$ and 36 -year-old Michelle ${ }^{1}$, were forced into the trunk of the car, driven to a dark, wooded area, and were subsequently raped. They were left tied to trees as the perpetrators drove away. The victims in the house untied themselves in time to record the license plate number of the perpetrators' car for police. Isabelle and Michelle also eventually managed to untie themselves from the trees and sought help from a nearby home. Soon after the crime, the police showed the victims photographic lineups for two suspects. Michelle identified both Douglas James and Alan Crotzer, while one of the other victims also identified Douglas. The next day, police showed the victims more photos, and three of them identified Corlenzo James, the brother of Douglas, as the third perpetrator. During their subsequent trials, Corlenzo pled guilty to robbery and assault, Douglas defended himself by claiming the rape was permitted by the adult victim (i.e., consent defense), but Alan maintained his innocence and claimed he had no knowledge of the crime. All three were convicted, with Crotzer given a prison sentence of 130 years (Innocence Project, 2017).

Unfortunately, the victims in this case were only correct about two of the three perpetrators. In 2003, new analyses of the DNA found on the rape victim's clothing definitively excluded Alan Crotzer as a rapist. Despite the fact that all five eyewitnesses made in-court identifications of him, Alan was an innocent man convicted of a crime he did not commit. Douglas eventually revealed the third perpetrator to be a childhood friend and admitted that they had never met Alan before he was accused of their crime. In 2006, after 24 years in prison, Alan was finally released.

Although we know a lot about eyewitness memory in general- four decades of research provides a number of insights as to what might have produced a faulty memory of Alan Crotzer - it is fair to say that we do not know much specifically about eyewitness memory for multiple perpetrator crimes. We do know that multiple perpetrator crimes are prevalent around the world and that such crimes are among the most difficult to solve (Dauvergne \& Li, 2006; Liem et al., 2013). We also know that nearly two-thirds of surveyed U.K. police report issues and confusion in conducting multiple suspect identification procedures, that eyewitness identification accuracy tends to decrease as the number of perpetrators increases, and that adapted lineup procedures attempting to address this

\footnotetext{
${ }^{1}$ Not their real names
} 
identification have had limited success (Hobson, Wilcock, \& Valentine, 2012; Horry, Halford, Brewer, Milne, \& Bull, 2014).

Thus far, research has done little to uncover or explore those factors that are unique to eyewitness memory in the context of multiple perpetrator crimes. This leaves us with an incomplete picture when trying to understand eyewitness memory and decisionmaking in multiple suspect lineups. Furthermore, there is a paucity of information regarding how police construct identification procedures for a multiple perpetrator crime, to what extent those procedures are similar or different to lineups used for a single perpetrator crime, and what, if any, issues police or eyewitnesses face in this applied context. Thus, while there have been some attempts to create novel identification procedures adapted specifically to the context of multiple perpetrator crimes, it is unclear which theories in memory and decision-making should drive such attempts, whether the theories used for previous attempts are relevant to multiple perpetrator crime identification, and to what extent the traditional control groups in empirical studies reflect actual police methods.

Across five empirical studies and one exploratory survey, this thesis reviews police practice in three European countries in the context of multiple suspect identification (Chapter 2: Police Survey), tests the independence of multiple identification decisions made successively (Chapter 3: Experiments 1, 2 and 3), and examines the purported utility of face context cues for recognizing the faces of multiple perpetrators (Chapter 4: Experiments 4 and 5). This thesis seeks to extend a small, but growing, evidence base in eyewitness memory for multiple faces and eyewitness identification for multiple perpetrators.

The purpose of this chapter is to place this thesis in context by (1) establishing applied questions for research in multiple perpetrator identification, (2) reviewing the extant literature that has thus far adapted traditional lineup techniques for multiple perpetrator crimes, and (3) exploring the theoretical background for two factors in memory and decision-making that are both unique to multiple perpetrator crimes and have yet to be empirically tested. These two factors - the associations between multiple decisions and the associations between memories for multiple faces - are the focus of this thesis.

\section{IDENTIFICATION OF MULTIPLE PERPETRATORS}

Lineups: The applied eyewitness experiment. The case described at the outset of this thesis is just one example of the many crimes that are committed by multiple perpetrators - crimes that are often violent, premeditated and goal-driven. Multiple perpetrator crimes often involve victims or bystanders as eyewitnesses who may be asked to identify multiple suspects related to the multiple perpetrators. A lineup, with one suspect and at least five known-to-be-innocent look-alikes (called fillers), can be constructed using photographs or videos of the lineup members to show the eyewitness, or by having lineup 
members physically present (i.e., behind a one-way mirror). This lineup can be seen as an applied scientific experiment with tangible and immediate consequences for the lives of the suspect, the victim(s), and the wider community in which they live (Wells \&, Luus, 1990). In this 'experiment', police test the hypothesis that the suspect is the actual perpetrator of the crime and the eyewitness can either confirm the police hypothesis by choosing the suspect, or they can falsify the police hypothesis by rejecting the lineup (i.e., saying the perpetrator is not there) or by mistakenly identifying the known-innocent filler. Yet the outcomes of an experiment are only as valid as the methodology is sound. In this vein, it is critical to understand how factors outside of the control of the justice system (e.g., the presence of multiple perpetrators) and factors within the control of the justice system (e.g., instructions to witnesses with multiple lineups) might influence an eyewitness's memory and decisions in eyewitness identification procedures. We can use this knowledge to improve identification procedures for multiple perpetrator crimes or to make informed decisions about the validity of the evidence after the fact.

There is an extensive history of police practice inspiring empirical tests for eyewitness memory (e.g., witness instructions: Malpass \& Devine, 1981; post-identification feedback effect: Wells \& Bradfield, 1998), but only one such experiment specifically for multiple perpetrator identification procedures (Hobson \& Wilcock, 2011) ${ }^{2}$. One source of information on current practice is surveys of police practitioners, such as the most recent National Institute of Justice survey of police practice across the United States (Police Executive Research Forum, 2013). However, there is only one police survey of law enforcement agencies that asked about police procedures specifically in the context of multiple perpetrator crimes (Hobson et al., 2012).

Multiple suspect lineups in practice: U.K. police survey. In their survey of 29 lawenforcement agencies in England, Wales, and Northern Ireland, Hobson and colleagues (2012) were the first to ask police officers specifically about characteristics of encountered multiple perpetrator crimes and identification practices in the case of multiple suspects. The only instructions that police receive on multiple suspect identification procedures come from the Police and Criminal Evidence Act (PACE, 1984), which is U.K. legislation that includes detailed directives on appropriate procedures for collecting eyewitness evidence. Specifically, PACE instructs that multiple suspects for a single perpetrator crime be presented in separate identification lineups (Code D, 2011). According to this format of presentation, an eyewitness must make a decision about the first lineup for the first suspect and is only presented the second lineup if they have rejected the first. For multiple suspects

\footnotetext{
2 While other experiments have tested novel lineup procedures for multiple perpetrator crimes, Hobson and Wilcock (2011) is the only published experiment specifically comparing procedures to known police practice for multiple suspect identification administration.
} 
of a multiple perpetrator crime, however, PACE dictates that "Only one suspect shall be included in an identification [lineup] unless there are two suspects of roughly similar appearance," (PACE Code D, 2011, Annex A, p. 47). This means that while PACE rules restrict multiple suspects to appear in the same lineup for single perpetrator crimes, they make an exception for multiple perpetrator crimes.

In light of sparse instructions on multiple suspect identifications, it is no surprise that nearly two-thirds of officers report frequently running into difficulty when administering multiple suspect lineups. While all officers reported creating separate lineups for separate suspects, challenges arose in situations outside of the PACE protocol. In particular, having to adapt instructions, receiving complaints of "blindness" from eyewitnesses viewing too many faces, and being uncertain whether to accommodate outof-ordinary requests (i.e., an eyewitness wants to view all lineups before making any identification decisions). Another concern is eyewitnesses attempting to choose multiple suspects from the same, single-suspect lineup. In such cases, it is unclear how to proceed, and officers provided diverging responses on whether eyewitnesses should be permitted to view the following lineups.

This survey represented an important initial step to understand multiple suspect identification procedures in practice. However, there are some concerns that are unique and inherent to multiple perpetrator identification procedures that were not yet addressed. These include if and when multiple lineups are administered (i.e., as suspects become available, or only when all suspects are available), who is responsible for constructing the multiple lineups (i.e., whether one person is responsible for all lineups of a multiple perpetrator case), and how officers perceive the utility of witnesses who identify some, but not all, of the suspects. One aim of this thesis is therefore to expand our knowledge on police practice in confronting multiple suspect identification procedures. In a new police survey (see Chapter 2), the original questions from Hobson and colleagues (2012) were adapted and translated into two languages (Swedish and Dutch) along with new questions to address the concerns above, to be distributed in Sweden, Belgium, and the Netherlands. Such information can help to guide experimental research in eyewitness identification for multiple perpetrators, set results of that research back into its applied context, and help to eventually evaluate and inform police practice in multiple perpetrator cases.

Multiple perpetrator lineups in the lab. Although we know little about multiple suspect identification procedures in practice, experimental research has eked out an initial picture of the challenges in identifying multiple perpetrators. In laboratory experiments, mock-eyewitnesses are consistently less accurate in identifying actual perpetrators and rejecting innocent suspects from lineups if they viewed multiple perpetrators vs. a single perpetrator. When participants viewed a non-violent event with one, three, or five 
perpetrators, only $30 \%$ of participants in the three-perpetrator condition and $20 \%$ in the five-perpetrator condition made accurate identifications of the main perpetrator from a target-present lineup (compared with $40 \%$ in the single-perpetrator condition; Clifford \& Hollin, 1981). In another experiment, $54 \%$ of participants who saw a perpetrator alone were able to accurately identify him/her, while only $29 \%$ of participants who saw the perpetrator with an accomplice could do the same (Megreya \& Bindemann, 2012). This pattern holds when participants are asked to watch live mock crimes or study photos (Nortje, Tredoux, \& Vredeveldt, 2017), when the multiple perpetrators are of the same or different genders (Megreya \& Bindemann, 2012), and even when participants are permitted unlimited time to study the multiple perpetrators (Megreya \& Burton, 2006).

Three published studies and one unpublished thesis to date have attempted to address the multiple perpetrator identification disadvantage demonstrated above, with limited and varying success, through adapted identification procedures. First, Wells and Pozzulo (2006) introduced what they called a two-person serial lineup for a crime involving two perpetrators. After watching a video of a theft, participants viewed a series of suspect photographs two at a time - one photo (filler or suspect) for the assailant lineup was always paired with one photo (filler or suspect) for the accomplice lineup. Participants viewed a series of these coupled photographs until they had seen six photos for the assailant and six for the accomplice. Although the lineups for each were flashed side-by-side on the screen, the two suspects were never shown simultaneously; each suspect was always paired with a filler. In theory, the context of one face should aid the ability to recognize or reject the other face (see encoding specificity principle: Thomson \& Tulving 1970). Whereas this novel procedure did not produce significantly more correct identifications when a target was present, it did produce significantly more correct rejections when the target was absent. Implications of these results will be discussed later.

Another novel identification procedure which adapted current PACE procedures in the U.K. was proposed by Hobson and Wilcock (2011) for a three-perpetrator crime. Participants viewed sequential video lineups for the perpetrators, each one after the other (i.e., perpetrator 1- 2 - 3), but were not allowed to make any identification decisions. Then, they viewed the sequential lineups in reverse order (i.e., perpetrator 3 - 2 - 1) and were asked to make an identification decision before moving on to the next lineup. The authors hypothesized that this technique would aid source monitoring, or the process by which we determine the origin of a memory (i.e., whether the details of eyewitness reports are from own memory of the crime, discussions after the fact, or even from a dream (Johnson, Hashtroudi, \& Lindsay, 1993). They hypothesized that this technique allow the participants to anchor their memories of the event with the appropriate lineup before making any decisions, thus avoiding source monitoring errors in confusing which 
perpetrator performed which action during the crime. Meanwhile, the order in which the perpetrators were presented was intended to reduce the high cognitive load participants experience as a result of viewing all lineups. This novel presentation style produced an increase in correct identifications in target-present lineups for two out of the three perpetrators, without effect on correct rejections for target-absent lineups for any of the innocent suspects.

Next, Dempsey and Pozzulo introduced the elimination lineup for use with adults (2008) and children (2013) making identification decisions after witnessing a twoperpetrator crime. An elimination lineup is a two-step decision process whereby witnesses are initially asked to look at a simultaneous lineup with six photos and choose the person that looks most like the perpetrator. All other photos are then removed, and the witness is asked to compare his/her memory of the perpetrator to the chosen photo and determine if this person is the actual perpetrator. In this study, when the thief was absent from the lineup, the elimination lineup procedure produced significantly more correct rejections compared with a standard simultaneous lineup. When the accomplice was present in the lineup, the sequential lineup produced more correct identifications compared with to the elimination lineup.

Lastly, Dempsey (2012) conducted two experiments for two-perpetrator crimes, attempting to use the face of one perpetrator to cue the identification of the other. In both experiments, participants viewed a two-perpetrator crime and 5-10 days later were asked to identify one of the perpetrators. As a cue, they were provided either with the correct face of the second perpetrator (correct cue), a second face that they had never actually seen before (incorrect cue), or no face (no cue). In Experiment 1, accurate cues and no cues led to more correct identifications of the actual perpetrator compared with incorrect cues. However, there was no difference in performance between participants who received an accurate cue and those who received no cue at all. Correct rejection rates of innocent suspect lineups did not vary based on the cues. In Experiment 2, participants were given the same types of cues, but were also given either biased or neutral instructions. There were no differences between groups based on cue veracity.

In summary, the adapted sequential lineup (Hobson \& Wilcock, 2011) increased correct identifications for two out of three perpetrators, the elimination lineup (Dempsey \& Pozzulo, 2008; 2013) with adults increased correct rejections for the innocent suspects of both perpetrators, but with children increased children's correct rejections for one of the perpetrators and decreased accurate identifications for the other perpetrator. The other methods had no significant effect on correct identifications of actual perpetrators or correct rejections of innocent suspects. Although Dempsey's (2012) cued lineup experiments demonstrated that correct cues were associated with higher rates of correct identification, 
there was no meaningful difference between providing a correct cue and providing no cue. These results suggest that incorrect cues may have undermined identification performance, but that providing a correct cue did not enhance identification performance and therefore does not represent a benefit to the novel procedure. More importantly, these findings are difficult to explain and provide limited insight to apply to future research on multiple perpetrator identifications; when these new methods produce null or inconsistent findings, it is unclear if it is because (1) the proposed adaptations did not address the mechanisms they were intended to, or (2) the theories used to justify these adaptations are not relevant to the multiple perpetrator identification disadvantage.

For example, in the two-person serial lineup (Wells \& Pozzulo, 2006), the context of one target face should theoretically aid our ability to recognize or reject the other target face by taking advantage of the fact that memories are associative by nature. In other words, a stored memory trace of a person's face is integrated with other contextual memories, including details like time of day, location, other people present, other events that happened before or after, thoughts, colors, smells, and sounds (Thomson \& Tulving, 1970). When we retrieve any single one of these details from memory, other details are also likely to be remembered in conjunction. Therefore, providing one or more of these contextual cues could help to access the memory of the perpetrator's face. However, this contextual cue is useful only if it is accurate (Davies, 1988). Thus, when Wells and Pozzulo's (2006) lineup does not improve identification accuracy, it is unclear if it is because contextual cuing is not useful for faces in a lineup context or because the suspects are never shown together, and thus one face does not act as a cue for retrieving the features of the other face.

Another example is the adapted sequential procedure (Hobson \& Wilcock, 2011). This experiment made two modifications to the traditional sequential identification procedure: allowing witnesses to view all three lineups before making a decision and doing so while presenting the identifications in reverse order. This procedure raises three issues. First, there is no evidence that the primacy/recency effects are problematic in eyewitness identification for multiple perpetrator crimes. The second manipulation is to present the lineups in reverse order to address primacy/recency effects of memory-the phenomenon that the first (primacy) and last (recency) items of a study list are more easily remembered than those in the middle. It is assumed that presenting the lineups forwards naturally enhances eyewitness memory for the first lineup, and subsequently presenting them backwards benefits memory for the third lineup. Notably, this adaptation is an attempt to improve memory for the lineups themselves, not the perpetrators. It is unclear how accounting for primacy/recency effects of working memory of the lineups themselves would enhance identification accuracy for the perpetrators, particularly given that identification 
decisions are most accurate when automatic recognition occurs (i.e., "his face jumped out at me") compared with when comparative processes are engaged (i.e., of these options, he looked most like the perpetrator; Dunning \& Stern, 1994).

Second, there is thus far no evidence that these manipulations solve issues in source monitoring or priming/recency effects. The first manipulation was an attempt to reduce source-monitoring errors by allowing eyewitnesses to properly associate the perpetrators of the event with their respective lineups. It is apparent that source monitoring errors can occur in the realm of eyewitness identification; in particular, the misidentification of innocent bystanders (vs. actual perpetrators) is thought to be in part a result of a feeling of familiarity while viewing a lineup, but an error as to the source of that familiarity (Perfect \& Harris, 2003). One infamous example of this is when psychologist and eyewitness expert Douglas Thomson was accused of rape as a result of the victim's description of her attacker. However, Thomson was giving a live TV interview during the time of the rape and therefore could not have been the perpetrator. It was later established that the victim had been watching the TV interview before the attack and had misattributed her recollection of Thomson as that of the perpetrator (Thomson, 1988 as cited in Schacter, 1999). It could also be that an eyewitness saw a perpetrator and a bystander, knows that they are separate people, but mixes up their identities and therefore chooses the bystander from the lineup. Both cases involve the accurate recognition of a before-seen face, but the inaccurate classification of that person as the perpetrator after recognition. Source monitoring solutions do not target the recognition of an item (i.e., perpetrator), but rather their subsequent classification (i.e., role assignment) afterwards. This is not to say that providing context is useless. It is possible that providing contextual cues for the eyewitnesses enhances access to the memories for each of the perpetrators, thereby increasing identification accuracy (see discussion below). Meanwhile, primacy effects are moderated by variables at the time of encoding the targets (i.e., increasing or decreasing exposure to each of a list of stimuli) while recency effects are eliminated through increased delay between encoding and test (Bjork \& Whitten, 1974; Murdok, 1962). It is therefore unclear how presenting the lineups forwards and then backwards would impact either phenomenon.

Third, if source monitoring or primacy/recency effects are problematic for identification accuracy for multiple perpetrators, and if these manipulations do appropriately address these problematic issues, then the fact that both manipulations are implemented simultaneously obstructs our ability to disentangle the results to determine the separate effects of each manipulation. Even if the lineup procedures had successfully enhanced eyewitness identification accuracy for multiple perpetrators, research would still need to examine the underlying mechanisms of the procedure. Ultimately, it is difficult to 
interpret such results because research to date on identification lineup solutions consists of premature attempts to solve problems that are still not well understood. In other words, the adapted lineups amount to trial-and-error solutions. This is problematic because this uncertainty about the mechanisms underlying methodology makes it difficult to build on the lessons from previous experiments in subsequent research, whether or not those procedures used were successful. Meanwhile, other research in recognition and decisionmaking (reviewed below) provides a foundation for understanding what may impact eyewitness performance on these adapted lineup procedures.

\section{COGNITIVE MECHANISMS UNDERLYING THE IDENTIFICATION LINEUP: MEMORY AND DECISION-MAKING}

An identification decision is composed of two overlapping, but distinct, processes for the eyewitness: memory and decision-making. Memory is the representation of the perpetrator, like a picture in the mind's eye of the person to be remembered. Because memory is not a camera, it is likely for this representation to be degraded over time, and it is possible for it to be eventually forgotten, distorted by external information, or even replaced (Deffenbacher, Bornstein, McGorty, \& Penrod, 2008; Loftus, 2005). Decisionmaking refers to the choice made at the time of the identification procedure. Ideally, the decision to identify a suspect or reject a lineup is based on the actual memory of the perpetrator, even though that memory may have been degraded or distorted over time. However, it is entirely possible to make a decision in the absence of memory; an eyewitness may reject a lineup because they do not remember the perpetrator, or they may choose a suspect in spite of the fact that they do not clearly remember the perpetrator (e.g., Horry et al., 2014; Innocence Project, 2017). It is also possible to make a decision based on a wrong memory; an eyewitness may, for example, use an inaccurate memory and misidentify the innocent suspect as the perpetrator.

As illustrated above, both memory and decision-making affect the outcomes of identification decisions. Importantly, the presence of more than one perpetrator during the crime presents additional complications for encoding, recognition, and decision-making that are both unique and inherent to multiple perpetrator crimes, thus warranting particular consideration. For example, eyewitnesses who have divided their attention between perpetrators, resulting in weak memory for faces from the outset, may later be asked to make multiple recognition decisions for suspects in the same lineup or multiple identification decisions for suspects in multiple lineups. Thus, the eyewitness is storing multiple faces and making multiple recognition and identification decisions and it is unclear to what extent these memories and decisions are connected or influence each other. Thus, questions of particular interest in this field are: (1) are multiple identification decisions 
independent (i.e., does a previous identification decision impact following ones)? and (2) are memories for multiple faces linked and, if so, can this link be used to enhance recognition performance?

Non-independence of multiple decisions. To date, eyewitness researchers and practitioners have assumed that an eyewitness is making a decision based solely on the information related to that identification procedure, meaning that it is presumed that an eyewitness making multiple identification decisions is making a series of independent decisions. However, research in perception and memory demonstrates repetitive tasks produce patterns of decisions that are linked such that responses on a current trial may favor (assimilate) or disfavor (contrast) the preceding trials (Treisman \& Williams, 1984; Malmberg \& Annis, 2012). For example, in one experiment, participants were played a series of auditory tones and asked to categorize them as being loud or soft. If the previous tone was loud, then participants were more likely to also categorize the current tone as loud (Holland \& Lockhead, 1968). These so-called sequential dependencies can be found across any series of repetitive tasks, such as perceiving a series of dim light-flashes in a dark room (Did you see the light? Yes vs. No), classifying the emotions (from angry to sad) of ambiguous faces, and judging attractiveness (hot vs. not) of dating app profile photos (Howarth \& Bulmer, 1956; Taubert, Van der Burg, \& Alais, 2016; Zhou, Lapedriza, Xiao, Torralba, \& Oliva, 2014). Some evidence shows that sequential contrast effects (i.e., a form of sequential dependency) may arise in legally relevant decision-making, such as judges granting or denying asylum to refugees (Chen, Moskowitz, \& Shue, 2016).

Importantly, these dependencies have also been detected in participants making a series of recognition decisions for previously-studied lists of words, non-words, and landscape photos (Malmberg \& Annis, 2012). In this particular experiment, participants were asked to study a list of 166 words and were later tested on their memory for those words. They were presented with a series of trials including the words they had previously studied (old words) and words they had not studied (new words) and asked to determine whether the word was old or new. Results showed that participants were significantly more likely to respond "old" to a current trial if they had done so on the previous trial. This was independent from whether the previous response was correct. Even though judgments of recognition involve referencing a reservoir of memories that are not available for the previous examples like perceiving sounds and assessing attractiveness, sequential dependencies still arise in these series of decisions.

Eyewitness identification is a task that provides some theoretical resemblance to the decisions listed above. Particularly in the event of a multiple perpetrator crime, an eyewitness may be asked to make multiple, sequential recognition decisions for the suspects related to the various perpetrators. Given that the forensic context is one in which 
such recognition judgments may carry severe consequences, it is critical to understand the link that may exist between the multiple identification decisions. One aim of this thesis is therefore to establish the independence or non-independence of multiple identification decisions in the context of multiple perpetrator crimes.

Context in memory. Memory is the other integral portion of the identification process, and it is desirable to preserve eyewitness memory as much as possible in order to use memory evidence within an investigation. In particular, researchers seek to identify and therefore protect eyewitnesses from external influences on memory (i.e., post-event information; Frenda \& Loftus, 2011) and to provide tools to reliably elicit as much memorial information as possible from eyewitnesses (i.e., Self-Administered Interview; Hope, Gabbert, \& Fisher, 2011; Krix, 2015). This is not dissimilar from the way forensic scientists seek to safeguard DNA evidence from contamination, degradation over time, and develop new technologies to collect and test the available evidence.

Yet, the preservation of memory evidence is dependent upon the strength of that initial memory trace to begin with. The recognition and identification of an unfamiliar face from memory is a difficult task that becomes more challenging as the number of unfamiliar faces to be remembered increases (Bindemann, Sandford, Gillatt, Avetisyan, \& Megreya, 2012; Clifford \& Hollin, 1981; Megreya \& Bindemann, 2012; Nortje et al., 2017). This difficulty begins at encoding, where divided attention during the criminal event limits the richness of details that can be stored in the first place. Some evidence suggests that people are only able to process one face at a time; specifically, that humans suppress the processing of features of one face in order to accurately encode the features of the other (Bindemann, Burton, \& Jenkins, 2005; Bindemann, Jenkins, \& Burton 2007; Boutet \& Chaudhuri, 2001; Palermo \& Rhodes, 2002). However, divided attention does not entirely account for the multiple-face recognition disadvantage, because recognition accuracy drops when studying more than one face, even when participants are given unlimited amount of time to study those faces (Bindemann et al., 2012). In summary, it is clear that the capacity to recognize multiple faces is affected both by factors at encoding, such as divided attention, and at storage, such as the memorial demand of holding more unfamiliar faces in memory.

One means of aiding memory at retrieval is by providing some of the context that was available at encoding (encoding specificity principle; Thomson \& Tulving, 1970). In contextual cuing experiments, researchers may have participants study target persons against certain backgrounds, paired with job descriptions, or holding objects (see Davies, 1988 for a review). Then researchers provide, withhold, or substitute this available contextual information when testing memory for the target. For example, researchers may present participants with the target persons against the same background as previously studied, a different background, or no background at all. Typically, but not always, the 
correct context (i.e., same background) enhances recognition accuracy for the target person. Most pertinent to the context of multiple perpetrator identification, some researchers have successfully used other faces as contextual cues for recognition. Winograd and Rivers-Bulkeley (1977) and Watkins, Ho, and Tulving (1976) had participants study pairs of faces and later tested their memory for those faces. The expected pattern prevailed: participants were better at recognizing a target face if it was presented with the other face it had been paired with during encoding, compared with when a face was shown alone or with a different face.

This research suggests that other perpetrators of a multiple perpetrator crime could be used to cue recognition for other perpetrators. This is particularly relevant in cases where police may have multiple suspect lineups to administer to an eyewitness of a multiple perpetrator crime. However, there are several issues to address in order to determine whether face cues are useful in the forensic context. For example, although these studies typically show that context improves recognition of target faces, it could also include an increase in false alarms (i.e., incorrectly claiming to have seen a never-before-seen face). Additionally, although multiple perpetrator crimes can have more than two perpetrators present, past studies have only used pairs of faces at study. It is therefore unclear to what extent such cues could be useful when more than two targets are presented in a group to be studied. This thesis examines the utility of such contextual cues using new standards in methodological and statistical techniques (Simmons, Nelson, \& Simonsohn, 2011), and tests whether it is still useful when there are more than two faces to study at the same time.

\section{OVERVIEW OF THE CURRENT THESIS}

Despite the substantial number of multiple perpetrator crimes and the clear importance of understanding the fundamental questions addressed above, research into multiple perpetrator identification and recognition is scarce. Some experiments within the eyewitness identification paradigm use mock-crime stimuli with multiple perpetrators, but do not focus on variables specific to multiple perpetrator identification (e.g., appearance change instructions: Charman \& Wells, 2007; using registered intermediaries with children: Wilcock et al., 2017; modality of lineup: Egan, Pittner, \& Goldstein, 1977; Schiff, Banka, \& de Bordes Galdi, 1986). Other experiments make use of multiple faces in order to study issues tangential to multiple face recognition (i.e., associative memory: Watkins, Ho, \& Tulving, 1976; Winograd \& Rivers-Bulkeley, 1977; the brain's role in associative memory: Kan, Giovanello, Schnyer, Makris, \& Verfaellie, 2007; Preston, Shrager, Dudukovic, \& Gabrieli, 2004). However, fewer than 20 published experiments focus specifically on multiple perpetrator identification decisions (see Table 1.1 for list of experiments). It is clear 
that more research is needed to fill this gap in the field of eyewitness recognition and identification.

The current thesis does not use lineup procedures, however it comprises five chapters, including one survey of police practice and five experiments, to examine underlying issues in memory and decision-making that impact eyewitness identification in the context of multiple perpetrator crimes. Chapter 2 presents the results of an international survey of police practice for eyewitness identification in the context of multiple perpetrator crimes in Sweden, Belgium, and the Netherlands. Chapter 3 presents research testing the independence of multiple identification decisions made successively (Experiments 1, 2 and 3). Chapter 4 presents experiments examining the purported utility of associative memory for recognizing the faces of multiple perpetrators (Experiments 4 and 5). In Chapter 5, an overview of the key findings is presented, followed by theoretical implications for memory and decision-making research and practical implications for researchers and police involved with multiple perpetrator identification. These chapters are summarized in further detail below. 
Table 1.1

Published Experiments That Tested Eyewitness Memory for Multiple Perpetrators With Lineups

\begin{tabular}{|c|c|c|c|c|}
\hline \multirow[b]{2}{*}{ Author } & \multirow[b]{2}{*}{$N$} & \multirow[b]{2}{*}{$\begin{array}{l}\text { Encoding } \\
\text { modality }\end{array}$} & \multicolumn{2}{|c|}{ Number of targets } \\
\hline & & & Encoding & Test \\
\hline & & & 1 & 1 \\
\hline \multirow[t]{3}{*}{ Clifford \& Hollin, 1981} & 60 & Video & 3 & 1 \\
\hline & & & 5 & 1 \\
\hline & & & & 0 \\
\hline \multirow[t]{2}{*}{ Shepherd, 1983 (Expt. 4) } & 41 & Live & 2 & 1 \\
\hline & & & & 2 \\
\hline \multirow{2}{*}{ Megreya \& Burton, 2006 (Expt. 1) } & \multirow{2}{*}{20} & \multirow{2}{*}{ Photo } & 1 & 1 \\
\hline & & & 2 & 1 \\
\hline Megreya \& Burton, 2006 (Expt. 2) & 20 & Photo & 2 & 1 \\
\hline Wells \& Pozzulo, 2006 & 150 & Video & 2 & 2 \\
\hline Dempsey \& Pozzulo, 2008 & 132 & Video & 2 & 2 \\
\hline Kask \& Bull, 2009 (Expt. 2) & 225 & Photo & 4 & 4 \\
\hline Hobson \& Wilcock, 2011 & 72 & Video & 3 & 3 \\
\hline \multirow{2}{*}{ Megreya \& Bindemann, 2011} & \multirow{2}{*}{534} & \multirow{2}{*}{ Video } & 1 & 1 \\
\hline & & & 2 & 1 \\
\hline \multirow{2}{*}{$\begin{array}{l}\text { Bindemann, Sandford, Gillatt, Avetisyan, \& Megreya, } \\
2012 \text { (Expt. 2) }\end{array}$} & \multirow{2}{*}{24} & \multirow{2}{*}{ Photo } & 1 & 1 \\
\hline & & & 2 & 1 \\
\hline \multirow{2}{*}{ Bindemann et al., 2012 (Expt. 3) } & \multirow{2}{*}{32} & \multirow{2}{*}{ Photo } & 1 & 1 \\
\hline & & & 2 & 1 \\
\hline Dempsey, 2012 (Expt. 1) & 221 & Video & 2 & 1 \\
\hline Dempsey, 2012 (Expt. 2) & 341 & Video & 2 & 1 \\
\hline \multirow[t]{3}{*}{ Dempsey \& Pozzulo, 2013} & 90 & Video & 2 & 2 \\
\hline & & & 1 & 1 \\
\hline & & & 2 & 2 \\
\hline \multirow[t]{3}{*}{ Nortje, Tredoux, \& Vredeveldt, 2018} & 200 & Video & 3 & 3 \\
\hline & & & 5 & 5 \\
\hline & & & 10 & 10 \\
\hline
\end{tabular}

Police Survey on Multiple Perpetrator Identifications: Sweden, Belgium, and the Netherlands. A new, exploratory survey was developed to extend previous work by Hobson et al. (2012), which was translated into two languages (Swedish and Dutch) to be distributed to other European countries (Tupper, Sauerland, Sauer, \& Hope, 2018). This survey aimed to (a) inform our understanding of the prevalence and characteristics of multiple perpetrator crimes from the perspective of law enforcement agencies, (b) discern how agencies in various countries conduct identification procedures (e.g., lineups, photo-arrays, showups) with multiple perpetrators, and (c) gain insight into how law enforcement agents and eyewitnesses experience the identification process in the context of such crimes.

Experiments 1 and 2: Testing for sequential dependencies in eyewitness showup identifications. Research in perception and recognition demonstrates that a current decision (i) can be influenced by previous ones $(i-j)$, meaning that subsequent responses are not always independent. In Experiments 1 and 2, we examined the relation of previous 
identification decisions to subsequent choosing behavior in the context of the multiple showup (i.e., a one-person lineup) identification decisions for a multiple perpetrator crime. That is, if it is possible to predict current choosing on a showup identification decision from previous choosing, it would provide initial evidence that sequential effects may be present in multiple showup identification decisions. In both experiments, participants watched a mock-crime film involving three perpetrators and later made three showup identification decisions, one showup for each perpetrator. Because research has previously demonstrated that sequential dependencies in recognition are a result of interference from previous trials (Malmberg \& Annis, 2012), Experiments 1 and 2 considered both previous signal (targetpresence: present vs. absent) and previous response (choosing: yes vs. no) as predictors of current choosing behavior (Matthews \& Stewart, 2009). Experiments 1 and 2 were similar in procedure, with the exception that Experiment 1 used four trial conditions of targetpresence such that the first two trials were consistent in target-presence of the stimulus (i.e., target-absent/ target-absent or target-present/ target-present) and the third was either consistent or similar. However, this decision did not allow us to test for separate effects of previous target-presence and previous response between the first two trials. Therefore, Experiment 2 extended Experiment 1 by using all patterns of target-presence. We expected that initial showup responses would predict choosing for subsequent showup responses. In other words, choosing on a previous showup identification would lead to choosing on subsequent ones, and rejecting on a previous showup identification would lead to rejection of subsequent ones. We also expected previous target-presence to exert a separate influence on the current identification decision, such that the previous target being present would predict current choosing and the previous target being absent would predict current rejecting (e.g., Matthews \& Stewart, 2009).

\section{Experiment 3: Testing for sequential dependencies in faces as a function of} number of trials. Although consecutive recognition decisions have been shown to produce sequential dependencies, such effects were found to be inconsistent within the eyewitness identification context. Experiment 3 examined whether methodological differences between the recognition and eyewitness paradigms used in previous research on sequential dependencies could account for the inconsistent findings presented in Experiments 1 and 2. This experiment therefore sought to replicate previous recognition research in sequential dependencies using word and landscape stimuli, and extend these effects to face stimuli. This experiment also examined whether the strength of these sequential dependencies changed as a result of the number of test trials (i.e., beginning vs. middle of experiment). Participants studied pairs of words, landscapes, or faces, and were later tested for recognition. We expected that sequential dependencies would arise in all categories of 
stimuli, but that the strength of this effect would be weaker in the beginning compared to the middle of the experiment.

Experiments 4 and 5: Cued face recognition: Testing a tool to enhance eyewitness performance. In contrast to the previous experiments, Experiments 4 and 5 focused on the association between memories for multiple faces. The presence of multiple faces increases memorial demand, but also provides a naturally-occurring contextual cue that may promote recognition. Early experiments demonstrated the benefits of cues on face recognition when faces were studied as pairs (e.g., Watkins, Ho, \& Tulving, 1976), and some more recent experiments have tried to apply such techniques in the context of eyewitness identification when multiple perpetrators are involved in the crime (e.g., Wells \& Pozzulo, 2006). However, there is a paucity of contemporary research systematically examining cued face recognition effects in applied contexts. Experiments 4 and 5 sought to replicate previouslyreported enhancing effects in cued face recognition, to investigate the mechanisms underlying those effects, and to determine whether such effects could include more than two faces, as many crimes involve more than two perpetrators. To do this, we included the traditional condition in which participants study paired faces, and added both a control condition in which participants studied single faces and another experimental condition in which participants studied groups of four faces. At test, participants in the single-face condition were tested only on those individual faces without cues. Participants in the two and four-face conditions were tested using no cues, correct cues (a face previously studied with the target test face), or incorrect cues (a never-before-seen face). We hypothesized that correct cuing would enhance recognition accuracy for target faces compared to no cue and incorrect cues, but that this effect would be stronger for participants studying two faces compared to participants studying four faces at a time (see Chapter 4 for more detailed explanation). 
CHAPTER 2 
CHAPTER 2

\section{Eyewitness identification procedures for multiple perpetrator crimes: A survey of police in Sweden, Belgium, and the Netherlands}

Tupper, N., Sauerland, M., Sauer, J., \& Hope, L. (2018) Eyewitness identification procedures for multiple perpetrator crimes: A survey of police in Sweden, Belgium, and the Netherlands. Manuscript submitted for publication. 


\begin{abstract}
Despite the considerable proportion of crimes that involve multiple perpetrators, little is known about how police officers construct, administer, and record eyewitness identification procedures for multiple suspects. An online survey of law enforcement agents in Sweden, Belgium, and the Netherlands $(N=58)$ was conducted to obtain police perceptions of prevalence and characteristics of multiple perpetrator crimes, and to examine identification procedure practices given little to no guidance is provided to police. Practice converges in the use of sequential, photographic lineups, but diverges between and within countries on whether or not suspects of multiple perpetrator crimes should be placed in separate lineups. Results specifically highlight the role of context as one critical area for future research in identification for multiple perpetrator crimes (i.e., placing multiple suspects in the same lineup and asking eyewitnesses to look for a specific suspect).
\end{abstract}


Multiple perpetrator crimes are prevalent worldwide: Gang violence, hate crimes, rapes, and assaults are a few of the examples of violent crimes committed by groups (Hobson, Wilcock, \& Valentine, 2012; Horvath \& Kelly, 2009; Juodis, Woodworth, Porter, \& Ten Brinke, 2009; Sandholtz, Langton, \& Planty, 2013). Witnesses and victims to such crimes may provide perpetrator descriptions to investigators and later be asked to confirm or exculpate police suspects for the crime in an identification procedure. Because multiple perpetrator crimes present additional complications for police officers administering identification procedures, it is critical for the justice system to know the procedures police officers employ that may impact the accuracy of witnesses' or victims' identification decisions.

Despite increasing rates of multiple perpetrator crimes (e.g., Cooper \& Smith, 2011; Statistics Canada, 2016) and the difficulty police have solving these crimes (Dauvergne $\& \mathrm{Li}, 2006)$, there is a paucity of research examining eyewitness identification for multiple perpetrators. In particular, what little we know about eyewitness identification procedures for multiple suspects comes from a single survey of U.K. police (Hobson, Wilcock, \& Valentine, 2012). This is in stark contrast to an extensive literature surveying police practice for eyewitness identification procedures in general. Indeed, it is common to empirically test modified procedures against current practice, resulting in updating recommendations for procedures (e.g., Police Executive Research Forum, 2013). Meanwhile, crimes with multiple perpetrators are inherently different from crimes with a single perpetrator, including the prerequisite of there being multiple suspects. Such differences later affect the prosecution and sentencing of the alleged perpetrators. For example, in the context of a multiple perpetrator crime, police may have multiple suspects who may or may not include any of the multiple perpetrators, to whom they must attribute distinct perpetrator actions (i.e., who did what). Imagine a scenario where a victim is approached by four people. One person hits the victim, another steals their wallet, and the two other people in the group stand by and watch. The police may apprehend four suspects, any one of whom might be the person who assaulted the victim, took the wallet, or stood passively by. An eyewitness of a single perpetrator crime may only need to have an automatic recognition linking a suspect to the perpetrator (i.e., his face just popped out at me; Dunning \& Stern, 1994). In contrast, to fully prosecute the perpetrators of multiple perpetrator crimes, an eyewitness must not only recognize a suspect as one of the perpetrators (i.e., his face just popped out at me), but also discriminate which suspect relates to which perpetrator (i.e., he was the one with the red shirt), and further distinguish their actions in the crime (i.e., he was the one who took my wallet).

Moreover, police need to make practical decisions above and beyond those made in a single perpetrator crime. For example, with multiple suspects, they could ask the 
eyewitness to view a single lineup with the multiple suspects in it, or multiple lineups for the multiple suspects, or both. Such decisions may enhance or undermine the eyewitness' ability to make accurate recognition decisions and role attributions.

Unfortunately, there is not only limited research on eyewitness performance with multiple perpetrators, but also on how to administer lineup identification tasks in the case of multiple identifications. The dearth of research in the field of multiple perpetrator recognition and identification precludes the ability to make concrete recommendations on multiple perpetrator identification. It is therefore vital to understand what decisions police currently make in constructing, administering, and recording eyewitness identification procedures in multiple perpetrator crimes. This could not only allow us to place actual and experimental eyewitness accuracy in its appropriate context, but to guide research that would be relevant for policy in the field of multiple perpetrator identification. The current research attempts to fill this gap by surveying police on multiple perpetrator crimes and identification procedures in three European countries: Sweden, Belgium, and the Netherlands.

\section{MULTIPLE SUSPECT IDENTIFICATION IN THE U.K.}

One region that has at least minimal guidance on the conduct of identification procedures with multiple suspects is the U.K. The instructions outlined in the U.K.'s Police and Criminal Evidence Act (PACE; 1984) are as follows: "When all members of a similar group are possible suspects, separate identification parades shall be held for each," (PACE Code D, 2011, p. 181). This instruction might apply to a situation where a single member of a gang committed a crime, but the police do not know which one of the members is the alleged perpetrator. In this case, the eyewitness would view one lineup for the first suspect and make a decision about that lineup before viewing the next one. This is a logical extension of the PACE rules for single suspect lineups, whereby the established procedures are simply replicated separately for each one of the multiple suspects. For crimes with multiple perpetrators, the following adaption was added: "Only one suspect shall be included in an identification parade unless there are two suspects of roughly similar appearance" (PACE Code D 2011, Annex A, p. 47). In other words, while PACE rules forbid multiple suspects to appear in the same lineup for a single perpetrator crime, they make an exception for multiple perpetrator crimes.

According to the results of Hobson and colleagues' survey of U.K. police (2012), all 29 responding law enforcement agencies in England, Wales, and Northern Ireland create individual lineups for each suspect and require the witness to make a decision about the first before moving on to the next. However, officers also report frequently running into difficulty implementing such lineups, including having to adapt instructions, receiving 
complaints of "blindness" from witnesses having to view too many faces, and being unsure of how to handle out-of-ordinary requests from eyewitnesses. Specifically, one officer reported that although they normally follow PACE procedure, they would allow the witness to see all lineups before making identification decisions if the witness specifically requested to do so. Officers also reported that witnesses would sometimes try to pick multiple suspects from the same lineup, even when there was only one suspect in each lineup. When this happens, some officers insisted that the eyewitness could no longer see the following lineups, but most reported that they would show the other lineups anyway. In such a case, it is unclear how officers should proceed and neither current police protocol nor psychological research have addressed this concern.

While the 2012 survey was an important first step to understand police practice for multiple perpetrator identifications, the results are limited to the U.K. Furthermore, the survey did not address some of the concerns that are unique to multiple perpetrator identification procedures. These concerns include if and when multiple identification procedures should be administered (i.e., witnesses view lineups for perpetrators as suspects become available, or all at the same time), who constructs the multiple lineups (i.e., the same or different persons construct all lineups for a multiple perpetrator crime?), and how officers perceive witnesses that can identify some, but not all, of the presented suspects.

\section{MULTIPLE SUSPECT IDENTIFICATION IN SWEDEN, BELGIUM, AND THE NETHERLANDS}

To address these questions, we developed a new survey to extend this previous survey with respect to both content and sample. The original questions from Hobson and colleagues (2012) were adapted and translated into two languages (Swedish and Dutch) along with new questions to focus on the concerns outlined above. This survey was distributed online to police agencies in three European countries accessible to the authors: Sweden, Belgium, and the Netherlands. In the following introduction, we will briefly outline existing identification policies for these countries.

Sweden. While there are no laws regulating eyewitness identification procedures in Sweden, a report of updated guidelines called Witness Confrontations (Vittneskonfrontation) was published by the Swedish National Police Board (Rikspolisstyrelsen, 2005). This report outlines recommended procedures for photographic, live, and video confrontations. For example, it is recommended that lineups should include one suspect and a minimum of 6 , but preferably 8-10, known-to-be-innocent fillers that match the description of the perpetrator. According to the guidelines, sequential lineup presentation is preferred over simultaneous presentation, and police are instructed that 
eyewitnesses should be shown all members of the lineup, even in cases when the eyewitness already verbalized an identification decision before the end of the lineup. If the eyewitness does not make a decision after viewing each lineup member twice, the identification procedure is terminated. Specifically for multiple suspects, the report notes "If there is more than one suspect, it should be arranged so that there is one confrontationgroup for each suspect" (section 11.3.3, p. 17). The guidelines also state that eyewitnesses should be instructed that the perpetrator may or may not be present, they should be instructed that it is possible to reject, and that the lineup may be shown twice.

Belgium. National regulations could not be found for Belgium. Circular letters and informal guidelines specific to police forces are not publically available.

The Netherlands. Dutch police are trained in a certification program in consultation of the Handleiding Confrontatie (Lineup Manual; van Amelsvoort, 2013), a manual on administration of eyewitness identification procedures. This manual provides detailed explanation and references to support the construction and administration of showups, and of live, photographic, static video, and dynamic video lineups. Police officers generally use a centralized national computer program, called Kompol, on which they can construct photographic lineups, administer a sequential lineup with automaticallyrandomized positions, provide standardized instructions, and record all decisions. Lineups must have one suspect and at least 5 to 11 fillers and witnesses are only allowed to see each lineup member once. This manual also contains contradictory recommendations for handling multiple suspects depending on the chosen presentation format. Specifically, multiple suspects in live lineups should be presented in separate lineups (p. 173 and 189). However, the manual states that photographic and video lineups should combine the lineups so witnesses only view one total lineup (p. 251 and 221).

The current survey. Given the minimal instructions provided to police officers in the event of multiple suspect identifications, it is of interest to determine how police perceive multiple suspect identification procedures both in terms of logistics and perceptions of the identification procedure. In this survey, we therefore aimed to (1) understand the prevalence and characteristics of multiple perpetrator crimes from the perspective of law enforcement, (2) discern how agencies in various countries conduct identification procedures with multiple perpetrators, and (3) gain insight into how law enforcement professionals and eyewitnesses experience the identification procedure in the context of such crimes. We expect these to not only provide an initial picture of current practice, but to help elucidate important unresolved issues or questions for future research. 


\section{METHODS}

\section{SURVEY DEVELOPMENT}

We obtained the original version of the police survey from the authors (Hobson et al., 2012) with permission to adapt it. Eight of the original survey questions were used, two were adapted to form open questions, and 11 new questions were added. Survey questions can be found in Table 2.1. As part of the development of the survey, police contacts in each of the three countries to be surveyed received both the English and the appropriate translated version of the survey. These individuals were asked to provide feedback on the survey, including ease of understanding, the accuracy of terminology used in translations, the appropriateness and relevance of the procedures described, and to note if they felt any pertinent area was missing from the survey.

\section{SURVEY SECTIONS}

The survey included multiple choice responses and open-ended questions throughout five main sections. (1) In General Information (5 questions) we requested demographic information about the respondents, such as work experience and job role. (2) In Criminal Offenses ( 3 questions) we asked officers to report number of multiple perpetrator crimes they had encountered in the last 12 months and their characteristics. (3) In Current Procedures for Multiple Identifications (13 questions) we asked about known guidelines and implementation of creating, administering, and recording identification procedures for multiple suspects. (4) In Problems with Current Practice (2 questions) we asked officers to report on difficulties encountered during such identification procedures from the perspective of the administrator as well as the eyewitness. (5) Lastly, in Perceived Utility of Eyewitnesses of Multiple Perpetrator Crimes (2 questions) we elicited police perceptions pertaining to the performance of eyewitnesses of multiple perpetrator crimes, including compared to eyewitnesses of single perpetrator crimes. There was also space at the end for respondents to add comments on the current procedures. See Table 2.1 for a complete list of questions. 
Table 2.1

Questions for Police on Multiple Perpetrator Crimes and Eyewitness Identification Procedures

\section{General Information*}

1. Gender (male/female/other)

2. Age?

3. How many years of experience in conducting eyewitness identification procedures do you have?

4. What is your job role?

5. Jurisdiction?

\section{The Criminal Offences*}

1. Of the crimes have you dealt in the last 12 months, what proportion involved multiple suspect showings? (0$100 \%)$

2. How many suspects are typically involved in the multiple perpetrator cases you have dealt with? (Please select the box for the category that applies most often) (2-10)

3. In the past 12 months, what types of crimes have you dealt with that typically involve multiple perpetrators? (robbery / burglary/ assault/ sexual assault/ homicide/ other)

\section{Current Procedures}

Scenario $1^{* *}$

4a. Choose the option that resembles what you would do in this case (A1 or A2/ A1 and A2 separately/ A1 and A2 together)

4b. In your work with multiple perpetrator crimes, Scenario 1 occurs: (never/ sometimes/ often/ always)

Scenario $2^{* *}$

5a. Choose the option that resembles what you would do in this case (A or B/ A and B separately/ A and $B$ together)

5b. In your work with multiple perpetrator crimes, Scenario 2 occurs: (never/ sometimes/ often/ always)

6. In what manner do you present the parades to witnesses in a multiple suspect identification? Select all the options that apply: (Lineups: live/ photo/ video; Format: simultaneous/ sequential/ other; Show-ups: live/ photo/ video)

7. Are there any procedural requirements or guidelines in place for multiple suspect identifications?

8. How do you organize the identification presentations for eyewitnesses in the case of a multiple-perpetrator crime? (witness views when: all lineups available/ as lineups become available/ other)

9*. What instructions do you give to a witness for multiple perpetrator identifications?

$10^{*}$. Do you ask the witness to look for a specific suspect?

$11^{*}$. Do you ask the witness to describe the role of the suspect they are identifying?

12. Do you record all eyewitness identification decisions in a crime with multiple perpetrators?

13. Do you record confidence for all suspect identifications for multiple suspect identifications?

14. Who is responsible for constructing the lineups? Is the same person responsible for all suspect lineups in a given case involving multiple perpetrators?

\section{Issues with Current Practice*}

15. Do you, as someone who administers identifications, experience any problems with multiple suspect identifications?

16. Do you think witnesses experience any problems with multiple suspect identifications?

\section{Perceptions of Eyewitnesses}

17. How do you think eyewitnesses of a multiple perpetrator crime perform in identifications compared to eyewitnesses of a single perpetrator crime? Generally eyewitnesses to crimes committed by multiple perpetrators are as good as/ better)

18. In your opinion, how useful is a witness for you if they identify one, but not all of the suspects presented?

Your suggestions*

19. Do you have any ideas about how multiple suspect identifications could be improved from the point of view of the police?

* indicates original survey question from Hobson, Wilcock, and Valentine (2012)

** see Figure 2.3 for graphic illustration of scenarios 


\section{RECRUITMENT}

In agreement with police contacts, we used the snowball sampling method of recruitment, meaning that we used initial police contacts in each country to recruit colleagues and distribute the online survey link in a way that best suited the structure of the police in that particular country. Participants received the survey link via an e-mail, which also included a short explanation of the purpose and contents of the survey. Participants accessed the survey through Qualtrics (Qualtrics, 2018), where they read a more detailed information sheet, provided informed consent, and completed the survey questions.

Participants from Sweden completed the Swedish-translated version of the survey and participants from Belgium and the Netherlands completed the Dutch-translated version of the survey ${ }^{3}$. Officers answered the survey in their native language, but their responses were translated to English by native Dutch and Swedish speakers for the purpose of analysis.

This survey was approved by the ethical review board of the Faculty of Psychology and Neuroscience of Maastricht University (ECP-160_02_01_2016). Respondents provided consent by clicking the button to continue the experiment.

\section{RESULTS}

We did not conduct statistical comparisons between countries or procedures, but rather sought to describe the pattern of practice across countries. Due to attrition throughout the survey and due to active participants skipping questions, not all questions have the same number of respondents. The number of respondents still in the survey $(n)$ are reported with descriptives, and all percentages represent the proportion of a given response from the remaining respondents. See results in supplementary materials.

\section{GENERAL INFORMATION}

In total, we received responses from 72 law enforcement officials, however, 18 respondents did not continue past the general information section, and thus they were excluded from all analyses. This left us with an initial sample of 51 respondents from Sweden ( $n=17)$, Belgium $(n=20)$, and the Netherlands $(n=14)$ who had experience administering identification procedures to eyewitnesses. Survey respondents were first asked to provide general information about age, experience administering lineups, job role, gender, and jurisdiction of police work. While a majority of respondents across countries reported being an investigator (62\%; $N=51$ total respondents), other job roles included identification officer, detective, analyst, and intelligence officer. Ranges and means for age and experience in identification administration are reported in Table 2.2.

\footnotetext{
${ }^{3}$ The survey was not translated to French. Therefore, we did not target recruitment from Belgian regions that are primarily French speaking (e.g., Brussels).
} 
Table 2.2

Range of Age and Job Experience (Mean) for Police Respondents on Multiple Perpetrator Identification Survey

\begin{tabular}{lllr}
\hline & $n$ & Age & Lineup Experience (Years) \\
\hline Sweden & 17 & $27-61(M=38.64)$ & $1-25(M=6.47)$ \\
Belgium & 20 & $27-55(M=42.65)$ & $0.5-30(M=15.05)$ \\
Netherlands & 14 & $36-68(M=53.00)$ & $1-30(M=13.33)$ \\
\hline
\end{tabular}

Note. $N=51$

\section{THE CRIMINAL OFFENCES}

Next, officers were asked to report their estimation of the number and types of crimes they encountered that involve multiple perpetrators, as well as the number of perpetrators those crimes typically involved. On an 11-point scale (0 to 100\%), police officers on average estimated that just over $30 \%$ of cases they encountered in the last 12 months ( $n=48, M=4.38,95 \% \mathrm{Cl}[3.66,5.09])$ involved multiple perpetrator crimes, though estimates varied across a range of $0-90 \%$ (see Figure 2.1). Police officers overwhelmingly indicated that such crimes were most likely to involve 2-3 perpetrators. In total, only three police officers indicated that crimes were most likely to involve 4-5 perpetrators. Figure 2.2 breaks these crimes into estimates of frequency of multiple perpetrator crimes by category. Officers estimated the number of multiple perpetrator cases in the last 12 months for each crime category. The most common multiple perpetrator crime was estimated to be burglary (32\% of reported cases), assault (27\%), and robbery (25\%). "Other" responses included theft and attempted murder, cultivation of cannabis plantations, public violence, abuse, and trafficking. 


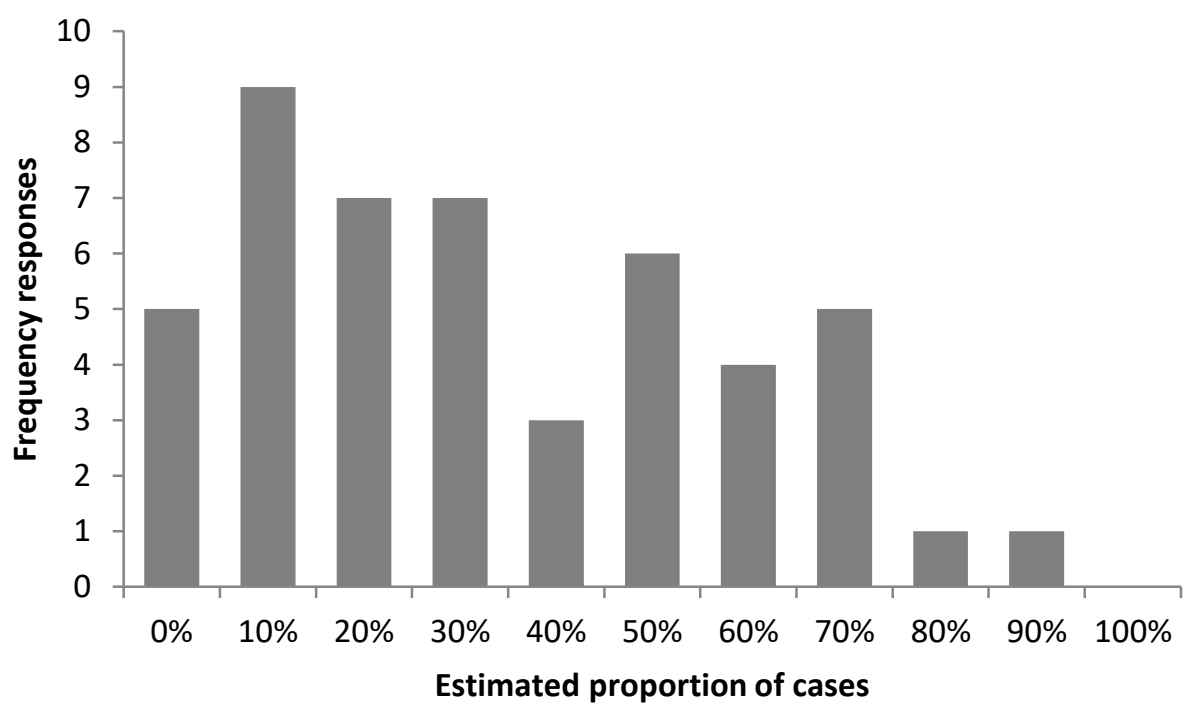

Figure 2.1. Estimated proportion of cases that included multiple suspect showings last 12 months. Note. $n=48$

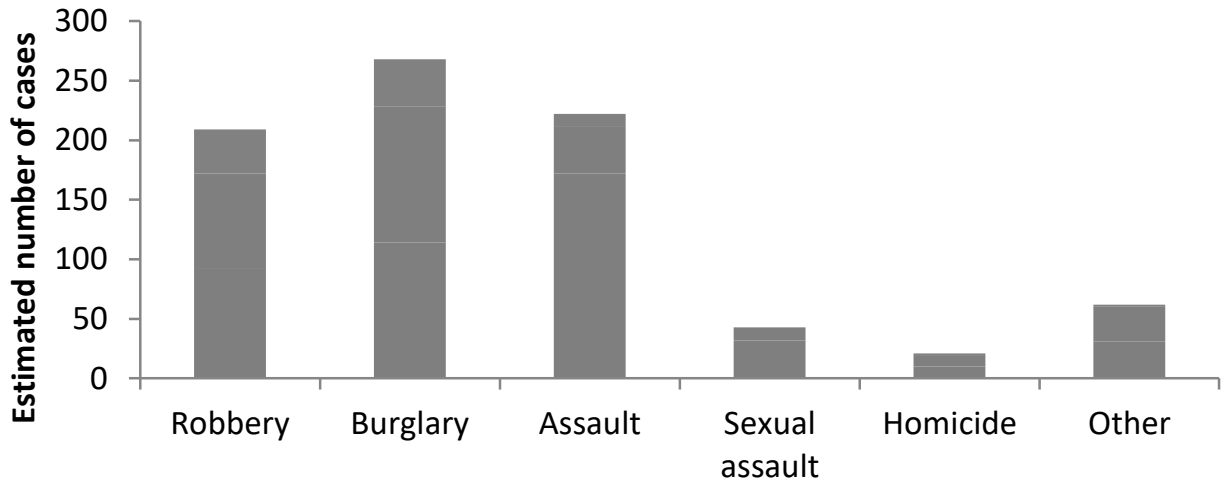

Figure 2.2. Estimated number of multiple perpetrator crime cases by category in the last 12 months. Note. $n=48$

\section{CURRENT PROCEDURES FOR MULTIPLE IDENTIFICATIONS}

Respondents were also asked to describe current procedures for constructing, administering, and recording eyewitness identification procedures specific to multiple perpetrator crimes. This was done to expand knowledge regarding actual practice outside of what minimal instructions are available for multiple suspect identification procedures in each country. Many of these were open questions (see Table 2.1 for specific questions), and thus many officers responded about lineup practice in general. These responses are 
included when relevant to the current research and quantitative responses are available in supplementary materials.

Guidelines for administration. Respondents were asked whether there were procedures or guidelines in place for multiple suspect identifications. This question received 28 total responses, of which nine affirmed guidelines or provided references to the specific guidelines (i.e., Rikspolisstyrelsen, 2005; van Amelsvoort, 2013). One Swedish officer noted that guidelines were "constantly updated and it is unclear what applies". Fifteen respondents provided answers that demonstrated knowledge of rules for lineup construction and administration, including number of fillers, sequential presentation format, suspect placement, and blind administration, though only five mentioned rules specific to multiple suspect identification. Only one Belgian officer answered "no", while another specified that there were no legal requirements, but that lineups were retrospectively assessed for probative value by a fact-judge.

Lineup construction. Survey respondents were asked to comment on whether the same officer was responsible for constructing all lineups in a multiple perpetrator case. Swedish and Belgian officers reported diverging answers here. One Swedish officer, for example, stated "It is preferred that the same person is responsible for arranging all the confrontations, so that the conditions remain the same." Another Swedish officer reported "Not necessarily - there is nothing clearly expressed about who the responsibility of this task falls on." By contrast, respondents from the Netherlands unanimously reported that the same person is responsible for all lineups. This might be because a limited number of officers in the Netherlands are certified in eyewitness identification procedures and these certified officers are the only ones allowed to construct lineups. For example, in the year 2016, 180-200 officers in the Netherlands held the authority to construct identification procedures (A. van Amelsvoort, personal communication, 2016).

When asked to indicate format of lineups, $94 \%$ of respondents $(n=48)$ indicated using photographic lineups. Five Belgian and one Dutch respondent also indicated the use of live lineups. Two Belgian respondents indicated the use of video lineups, while three Dutch respondents indicated the use of a unique context-reinstatement video lineup (i.e., Chroma-key technique) that superimposes videos of the lineup members over the video of the crimes scene. Seventy-nine percent of the sample $(n=47)$ indicated sequential lineup presentation, while the rest indicated simultaneous lineup presentation.

Although there were no open questions specifically asking about lineup construction, one Swedish respondent mentioned that one lineup required one suspect and seven fillers, so multiple suspects would each also require seven fillers. One Dutch respondent similarly specified that each lineup for each suspect should use a minimum of five and maximum 11 fillers per suspect (in accordance with the training manual). However, 
another Dutch respondent qualified that, in the case of three suspects, the minimum number of fillers (i.e., 5) should be used so that the witness would not see too many faces.

Lineup presentation. Although we did not ask an open question regarding whether multiple suspects should appear in separate or combined lineups, several respondents of each country spontaneously provided this information in their responses. Consistent with Swedish best practice guidelines, two Swedish and one Belgian respondent reported that suspects should not appear in the same lineup. By contrast, and consistent with Dutch best practice guidelines, one Belgian and two Dutch respondents reported that multiple suspects should not appear in the same live lineup, but should be presented together in the photographic lineups.

Scenarios for multiple suspect lineups. To be able to distinguish between situations in which there are multiple suspects for a single perpetrator vs. multiple suspects related to multiple perpetrators, the survey included two scenarios to differentiate between these two instances (see Figure 2.3). The scenarios were described as follows:

Scenario 1: Two men (A and B) robbed a bank. An employee witnessed the robbery. Two suspects are arrested, both suspected for being perpetrator $A$. The suspects are called suspect $A 1$ and suspect $A 2$. You are preparing an identification lineup.

Scenario 2: Two men ( $A$ and $B$ ) robbed a bank. An employee witnessed the robbery. Two suspects are arrested, one suspect for perpetrator $A$ and one suspect for perpetrator $B$. The suspects are called suspect $A 1$ and suspect B1. You are preparing an identification lineup.

In both cases, officers were asked how they would construct lineups for multiple perpetrators with respect to three options ${ }^{4}$ :

Option i: The eyewitness sees one line up, only for Suspect 1 or only for Suspect 2, not both. Option ii: The eyewitness sees two line ups, one for Suspect 1 and one for Suspect 2.

Option iii: The eyewitness sees one lineup, with both Suspects 1 and 2 in the same lineup.

\footnotetext{
${ }^{4}$ The responses here have been altered slightly to accommodate both scenarios for illustration purposes.
} 


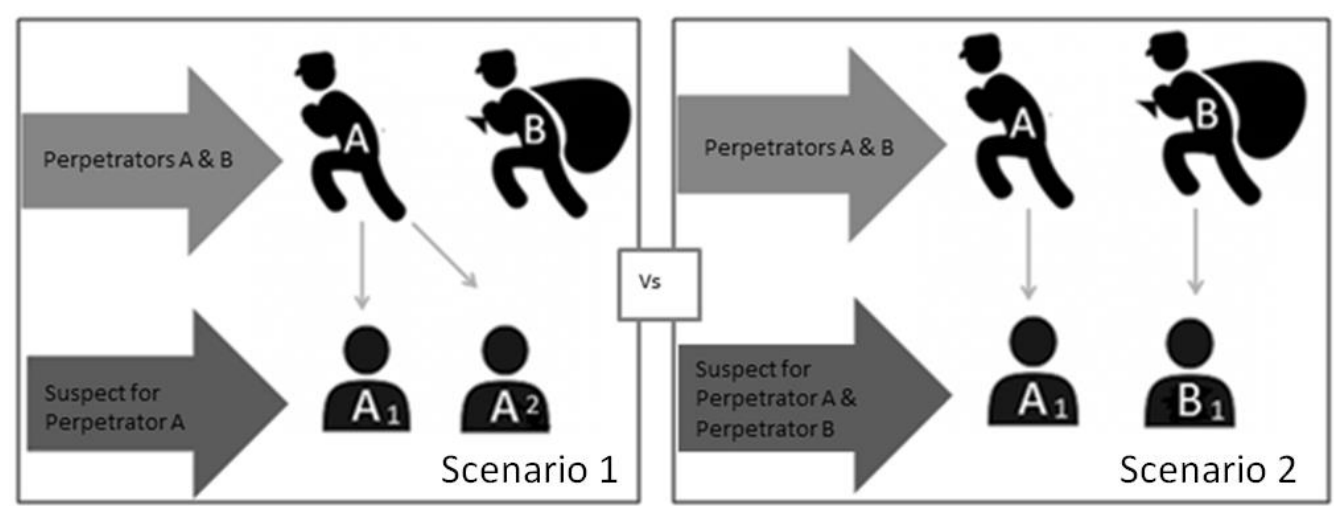

Figure 2.3. Illustration depicting Scenario 1 in which there is one perpetrator with multiple suspects and Scenario 2 in which there are multiple perpetrators with corresponding individual suspects. Scenarios are as follows. Scenario 1: Two men ( $A$ and $B$ ) robbed a bank. An employee witnessed the robbery. Two suspects are arrested, both suspected for being perpetrator $A$. The suspects are called suspect A1 and suspect A2. You are preparing an identification lineup. Choose the option that resembles what you would do in this case. Scenario 2: Two men (A and $B$ ) robbed a bank. An employee witnessed the robbery. Two suspects are arrested, one suspect for perpetrator $A$ and one suspect for perpetrator B. The suspects are called suspect A1 and suspect B1. You are preparing an identification lineup. Choose the option that resembles what you would do in this case.

As Figure 2.4 (Panel A) demonstrates, a majority of officers (54\%, $n=48,95 \% \mathrm{Cl}$ $[41.7,68.8]$ ) chose option ii for Scenario 1, meaning that the two suspects for the same perpetrator would be placed in separate lineups. By country, a majority of Swedish $(53 \%, n$ $=17,95 \% \mathrm{Cl}[29.4,76.5])$ and Belgian $(61 \%, n=18,95 \% \mathrm{Cl}[38.9,83.3])$, but not Dutch $(46 \%$, $n=13,95 \% \mathrm{Cl}$ [23.1-76.7]) officers chose this option. Many $(40 \%, n=48,95 \% \mathrm{Cl}[25.0,52.1])$ also chose option iii, for which the two suspects for the same perpetrator would appear in the same lineup (Sweden $=29 \% 95 \% \mathrm{Cl}[5.9,52.9]$; Belgium $=39 \%, 95 \% \mathrm{Cl}[16.7,61.1]$; Netherlands $=54 \%, 95 \% \mathrm{Cl}[23.3,76.9])$. Asked on the frequency of such a scenario, officers indicated that this situation occurs never or sometimes (Figure 2.4, Panel B). A majority of police officers $(65 \%, n=48,95 \% \mathrm{Cl}[50.0,79.2])$ also chose option ii for Scenario 2, for which the suspects of the two perpetrators would appear in separate lineups (Sweden $=71 \%, 95 \%$ $\mathrm{Cl}[47.1,88.2]$; Belgium = 78\%, 95\% Cl [55.6, 94.4]; Netherlands = 39\%, 95\% Cl [15.4, 61.5]). Again, many $(31 \%, 95 \% \mathrm{Cl}[18.8,43.8])$ also chose option $i i i$, indicating that both suspects for the two perpetrators would appear in the same lineup (Sweden $=18 \%, 95 \% \mathrm{Cl}[0,35.3]$; Belgium $=22 \%, 95 \% \mathrm{Cl}[5.6,44.4]$; Netherlands $=62 \% 95 \% \mathrm{Cl}[38.5,84.6])$. Scenario 2 was judged to be occurring more often than Scenario 1. 
A

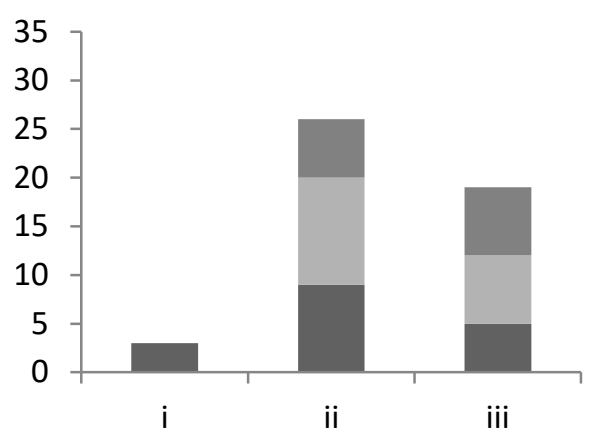

B

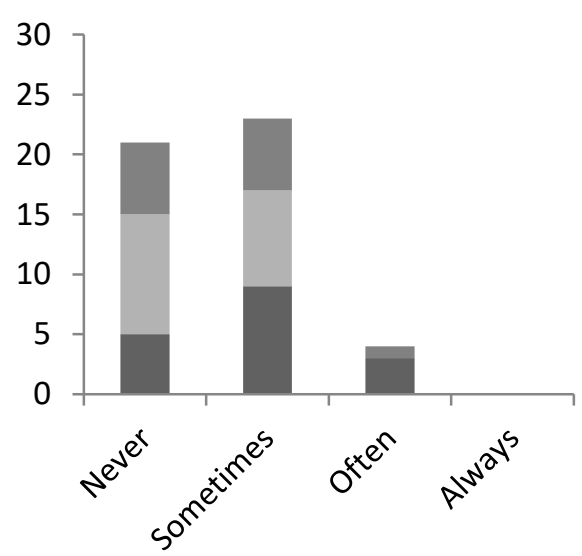

C

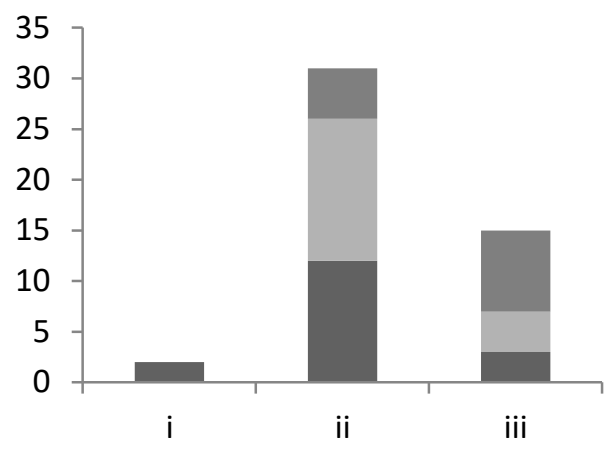

D

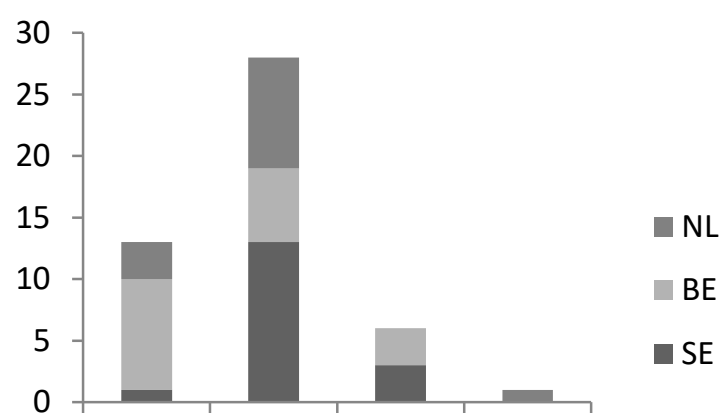

Figure 2.4. Top panels display police responses for what they would do in the events of Scenario 1 (Panel A: single perpetrator with multiple suspects) and Scenario 2 (Panel C: multiple perpetrators each with one suspect). Choices $A, B$ and $C$ refer to whether they would administrate lineups so that eyewitnesses see (A) Suspect 1 or 2 but not both, (B) Suspect 1 and 2 but in separate lineups, or (C) Suspects 1 and 2 in the same lineup. Bottom panels display how often police encounter this situation in administering lineups for Scenario 1 (B) and Scenario 2 (D).

When do witnesses see (a) lineup(s)? Survey respondents were also asked when eyewitnesses see multiple lineups. Specifically, when there are multiple suspects, do eyewitnesses see the lineups for the suspects as they become available, only when all lineups for the suspects are ready? They were also able to select the option "other" and were provided space to fill in their own response. Most Swedish (55\%; $n=11,95 \% \mathrm{Cl}[30.8$, 76.9]) and Belgian (71\%; $n=17,95 \% \mathrm{Cl}[50.0,88.9]$ ) respondents, but a minority of Dutch respondents (27\%; $n=11,95 \% \mathrm{Cl}[0,54.5])$, reported that witnesses are presented lineups as they become available. Thirty-three percent of Swedish respondents $(95 \% \mathrm{Cl}[7.7,53.8])$, 
$27 \%$ of Belgian respondents $(95 \% \mathrm{Cl}[11.1,50.0])$, and $46 \%$ of Dutch respondents $(95 \% \mathrm{Cl}$ $[18.2,72.7])$ reported that witnesses are presented lineups when all lineups are available. Swedish and Dutch respondents who answered "other" generally indicated that both were possible, but it would differ by case.

Instructions to eyewitnesses. Police officers were asked what specific instructions they give for lineups with multiple perpetrators. Only two Swedish officers reported instructions that were adapted to multiple suspects. One noted that instructions to witnesses specifically mention that multiple suspects would not be in the same lineup. However, another wrote that they would instruct the witnesses that it is possible that none of the suspects are present among the pictures, but that they would also falsely state to eyewitnesses, saying that several suspects could be present in the same series. Belgian officers did not mention altered instructions for multiple suspects or multiple lineups. Officers from the Netherlands reported written standardized witness instructions that are presented to eyewitnesses before the lineup and on the computer system during the lineup administration. One police officer stated that instructions for multiple suspects differ from those with one suspect, but another officer reported the instructions are the same regardless of how many suspects.

The next question on instructions appeared to confuse officers, specifically, "Do you ask the witness to look for a specific suspect (e.g., identify the one who was driving the car)?" This question was intended to examine whether officers inform the eyewitness which lineup is for which perpetrator before any identification decision is made. However, most officers who reported "yes" subsequently explained that they only did this following a positive identification. Although it is clear that this question did not elicit the responses expected, qualitative responses suggest officers do not generally instruct eyewitnesses to look for specific suspects. At least $90 \%(n=40)$ of all responding officers in some form indicated that role in the crime was only elicited after the identification procedure.

Recording decisions. Officers were asked whether they record all identification decisions. A majority of officers (90\%; $n=39,95 \% \mathrm{Cl}[82.1,97.4])$, reported that they record all identification decisions, whether or not the eyewitness chose the suspect. One Swedish officer wrote that it would be considered wrong, if not criminal, to not report all decisions. Only one of the four officers $(10 \%, 95 \% \mathrm{Cl}[2.6,17.9])$ who responded "no" explained that they only record positive identification decisions.

\section{PROBLEMS WITH CURRENT PRACTICE}

Police officers were asked whether, in the context of multiple perpetrator crimes, they or eyewitnesses encountered difficulties with lineups. Fifty-one percent $(n=39)$ reported experiencing problems for themselves, for witnesses, or both. Responses regarding lineup administration fell into two categories. The first was logistics related to 
materials. In particular, one common response is exemplified by one officer, who reported, "It is difficult to find the [fillers] for a lineup of several suspects. For two suspects you already need 10 [fillers] with a similar appearance to the suspect." Other concerns included the quality of photos available, with officers noting that outdated photos result in nonidentifications. The second category of answers concerned the difficulty in constructing appropriate lineups. For example, one officer expressed that it is difficult to follow all lineup recommendations at once, while another wrote that because there is not one assigned person who constructs all lineups "the quality often turns out highly varying, as different colleagues perform photo confrontations with different frequency." Another officer indicated that so many factors of lineup construction can influence eyewitness memory that it is unlikely to have a positive identification of the suspect. Notably, only one Dutch officer reported problems with administering multiple suspect lineups, namely the increased cost of making multiple lineups. Overall, Dutch police officers reported lineup administration to be well organized.

Responses regarding eyewitness difficulties generally concerned two categories: $16 \%(n=37)$ reported general memory issues not specific to multiple perpetrator crime, and $18 \%$ reported the tendency to mix up perpetrators or roles of perpetrators. One officer, for example, noted that it can be particularly difficult for eyewitnesses to separate memories for perpetrators when it was dark during the crime and the perpetrators of the crime look similar (i.e., gang members).

\section{PERCEIVED UTILITY OF EYEWITNESSES OF MULTIPLE PERPETRATOR CRIMES}

In the last section, officers were asked to indicate their subjective view on the utility of eyewitnesses of multiple perpetrator crimes compared with single perpetrator crimes. In particular, they were asked if eyewitnesses of multiple perpetrator crimes perform better, as good as, or worse than eyewitnesses of single perpetrator crimes. Most respondents felt that eyewitnesses of multiple perpetrator crimes perform "worse than" $(55 \%, n=39,95 \% \mathrm{Cl}[35.9,71.8])$ or "as good as" $(46 \%, 95 \% \mathrm{Cl}[28.2,64.1])$ eyewitnesses of single perpetrator crimes, but never "better".

They were also asked to comment on the investigative and/or probative value of an eyewitness that identified one suspect, but not the other(s). Most officers (87\%; $n=39$, $95 \% \mathrm{Cl}[76.9,97.4])$ reported that any identification is useful, even if other suspects were not identified; justifications for this included that one identification could provide more leads in a case, that one identification was better than none, and that this evidence was perfectly usable in court. One officer wrote, "It may well be that the witness is certain about it when they see one person and not about the other (s). It is actually very good that he adheres to the instructions. Because of this he seems just reliable." The remaining officers reported that the usefulness depended upon other factors, including other evidence, the 
circumstances of the crime. For example, some officers in Sweden and Belgium noted that failure to identify all perpetrators did not undermine the probative value of the identification as long as there was a sufficient explanation (e.g., the eyewitness had longer exposure to the perpetrator they identified, or that perpetrator was more distinctive, etc.).

\section{SUGGESTIONS FOR IMPROVED PRACTICE}

Suggestions made by Swedish and Belgian officers generally focussed on three categories: (i) calls to create a national database to make it easier to find fillers, including updated photographs, or 3D or video confrontations; (ii) calls for better training or having a specialist to consult for identification lineups. For example, one Swedish officer wrote, "Today there is no qualification needed to perform a witness confrontation, which is very strange since you need qualifications to do everything else in the agency. I have noticed that this leads to certain commonly accepted 'truths' that really are not true at all, for example, that live confrontations are always better,"; (iii) calls to move away from photo confrontations not least because of a perception that "prosecutors seem less and less interested in witness confrontations. The risk is too high that the witness will not identify anyone, despite us being sure the perpetrator is present in the material. In those cases the lawyer can use the confrontation to say that the witness says that the suspect hasn't done anything, he isn't identified" (Swedish officer). Meanwhile, in the Netherlands, many officers suggested returning to simultaneous identification procedures. For example, one officer wrote, "From my own experience, I think that the chance of identification will be greater than with a sequential confrontation. In case of multiple offenders and thus in case of multiple confrontation sequentially, the witness will see a lot of pictures. So what I suggest, in case of multiple suspects, is to again use the photo display board."

\section{DISCUSSION}

The current research sought to determine how police officers in three European countries experience multiple suspect identification procedures both in terms of logistics and perceptions of the identification process. We distributed an online survey to police contacts in Sweden, Belgium, and the Netherlands, with questions eliciting responses pertaining to the characteristics of encountered multiple perpetrator crimes, current guidelines, practice, and issues regarding multiple suspect lineups, and the perceptions of eyewitnesses of multiple perpetrator crimes. This survey extended previous research (Hobson et al., 2012) by including scenarios distinguishing between multiple suspects of a single perpetrator crime or multiple perpetrator crime, questions when lineups are conducted, who is responsible for the construction of the multiple lineups, and perceptions about the performance of eyewitnesses of single vs. multiple perpetrator crimes. Practice converges when it comes to the use of sequential, photographic lineups, and the collection 
of role assignment after the identification. However, practice diverges between and within countries on whether or not suspects of multiple perpetrator crimes should be placed in separate lineups, whether or not the same individual is responsible for all the lineups in a single case, and whether to administer lineups as suspects become available or only once all suspects are available. Results specifically highlight the role of context as one critical area for future research in identification for multiple perpetrator crimes (i.e., placing multiple suspects in the same lineup or asking eyewitnesses to look for a specific suspect). Findings are discussed in relation to Hobson et al.'s (2012) survey and the scientific literature on eyewitness memory and lineup administration.

Collapsed across countries, approximately one-third of respondents' cases in the last 12 months involved multiple perpetrator crimes. The average of our sample reflects the high-end of Hobson and colleague's (2012) results with U.K. police, two-thirds of whom reported between 10 and $30 \%$ of their caseload to include multiple perpetrator crimes. It is possible that this difference is a result of the different method of measurement between the surveys (i.e., our sample rated proportion of multiple perpetrator crimes on an 11-point scale vs. Hobson et al.'s 7-point scale), or reflects the greater range in responses (i.e., 0 to $90 \%$ in our sample vs. $<10 \%$ to $50-60 \%$ in the U.K.). Our results are also slightly higher than reported statistics on violent multiple perpetrator crimes, which typically range from 13$17 \%$ of homicides in the Netherlands, Sweden, and Norway and up to $33 \%$ of homicides in Canada (Liem et al., 2013; Statistics Canada, 2016). However, available statistics report only violent multiple perpetrator crimes such as homicides, and therefore do not include the non-violent incidents reported by our sample. This is particularly noteworthy given that the most common multiple perpetrator crime reported here was burglary. Other non-violent crimes included theft, cannabis cultivation, and trafficking. In line with Hobson et al., our results show that police are most likely to encounter multiple perpetrator crimes with 2-3 perpetrators, and that the most commonly-encountered multiple perpetrator crimes beside burglary were robbery and assault.

Police officers were also asked a series of questions regarding guidelines and procedures for multiple suspect lineups. Although nearly all police demonstrated some knowledge of evidence-based guidelines for identification procedures in general (i.e., that would also apply to single perpetrator crimes), few mentioned guidelines specific to multiple suspect identification procedure. However, responses do provide some clear trends. First, when asked to select from identification formats, respondents in all three countries overwhelmingly reported using sequential, photographic lineups. This is in line with current scientific research, which has traditionally endorsed sequential presentation producing more accurate identification decisions compared with simultaneous presentation as a result of a decrease in mistaken identifications (i.e., Steblay, Dysart, \& 
Wells, 2011); Police Executive Research Forum, 2013). This is also in line with national guidelines for Sweden and the Netherlands (Rijskreport, 2005; van Amelsvoort, 2013). However, it is worth noting that there is still debate within the field, with researchers opposing the assumed superiority of sequential presentation (i.e., Clark, 2012; Mickes, Flowe, \& Wixted, 2012). Another clear pattern was that nine in 10 officers insisted that they would ask for the eyewitness to designate the role only after they had identified a suspect. This is contrary to results from Hobson et al. (2012), who reported that U.K. administrators would generally inform eyewitnesses for which perpetrator the lineup was intended (e.g.,, the person that was holding the gun). While neither scientific recommendations nor national guidelines specifically address this topic, the decision to collect role assignment following the identification decisions does fit with the pattern of scientific guidelines aimed to prevent biasing the eyewitness' subsequent identification decision (e.g., double-blind procedures: Kovera \& Evelo, 2017) . On the one hand, asking the eyewitness to look for the man holding the gun may bias the witness to choose the innocent suspect who looks most like they would have gun, or, alternatively, to not choose the guilty suspect that actually had a different role in the crime. On the other hand, more than one in six police officers surveyed here reported that eyewitnesses were likely to mix up the perpetrators and the roles of the crime. Thus, providing contextual information like suspected role may be beneficial for the witness as they attempt to retrieve and appropriately distinguish between memories for perpetrators (see Davies, 1988, for a review). Further research is necessary to assess the merits, and potential consequences, of these alternative approaches.

Other questions produced divergent patterns of responses both within and between officers from Sweden, Belgium, and the Netherlands. Some appeared to be a result of systemic differences between countries. For example, while the Dutch officers unanimously reported the same person is responsible for constructing the multiple lineups within a multiple perpetrator case, Swedish and Belgian officers reported no clear rules designating one vs. multiple officers for this task. This is likely because the requirement for certification limits the number of Dutch police officers who are permitted to construct identification procedures, whereas the other two countries do not require officers to be certified specifically in identification procedures. Requirements to gain certification have the advantage that all officers constructing lineups have been specifically trained in evidence-based guidelines on eyewitness identification procedures. Incidentally, this also circumvents the concern that the quality of each lineup within a case fluctuates as a result of multiple officers creating the multiple lineups.

Other questions produced patterns of responses that varied regardless of country. For example, officers were divided on whether to wait for all suspects to present lineups to witnesses or to present them as they become available during the investigation. Again, 
there are no guidelines addressing this particular issue and empirical research provides conflicting concerns to balance: First, that memory degrades as the delay between the event and the identification lengthens, and that context aids recognition and recall (Davies, 1988; Deffenbacher, Bornstein, McGorty, \& Penrod, 2008). Thus, it is critical to provide an eyewitness with a lineup as soon as possible to ensure a stronger memory trace, resulting in more reliable evidence. On the other hand, because memory degrades at an exponential rate, context cues might be beneficial in providing memory support after the greatest drop in memory strength has already occurred. According to some calculations (Deffenbacher et al., 2008), an eyewitness may have no more than a 50\% chance of accurately identifying a perpetrator from a lineup after a one week delay. If a lineup is already delayed, it could be useful to further delay an identification procedure in order to use other suspects as a form of context reinstatement. Future research could examine the tradeoff between memory decay and contextual cues in the context of multiple suspect identification.

Other notable results of this survey were derived from the scenarios creating a lineup for multiple suspects in the context of a single perpetrator vs. multiple perpetrator crime. Across the three countries in which the survey was conducted, officers generally treated the two scenarios as equivalent; most often, officers reported separating the two suspects into individual lineups, but many alternatively chose to present them in the same lineup. Although the eyewitness identification literature consistently warns to separate multiple suspects of a single perpetrator crime (e.g., Wells et al., 1998), research has not satisfyingly addressed whether to separate or group multiple suspects of a multiple perpetrator crime during identification procedures (cf. initial tests: Kask \& Bull, 2009; Wells \& Pozzulo, 2006). Moreover, regulations within and across countries vary: police are instructed to separate suspects unless two suspects look similar in the U.K., to separate suspects for live lineups but not photographic lineups in the Netherlands, and to always separate suspects in Sweden. If an eyewitness is asked to make multiple identification decisions for multiple suspects, this means they may either make multiple identification decisions in succession, or make multiple identification decisions within the same lineup. In regards to the former, research thus far suggests that making multiple identification decisions is at least not harmful to eyewitness memory (Mansour, Beaudry, \& Lindsay, 2017). And indeed, the most important benefit of the single suspect lineup - the reduction in the probability that a suspect will be identified by chance - remains. However, research using contextual cuing recognition paradigms for typically reveals an increase in correct recognition of targets (Davies, 1988). Yet this increase in correct identifications may also be paired with an increase in false-identifications. It is noteworthy that Dutch police officers reported very few issues- for themselves or for eyewitnesses - in administering lineups. They also notably reported a lack of guidelines for only one of the questions posed (i.e., 
whether or not to provide lineups as suspects become available or once all suspects are available). This occurs in a country where a detailed, evidence-based manual is coupled with a certification program in order to conduct identification procedures. While it is possible police chose not to report issues, it is also likely that this system is useful in standardizing procedures across the country. This not only appears to support police in making decisions about lineup construction, but confirms the utility of psychological insight into lineup construction and administration (Sauerland, Krix, \& Merckelbach, 2016).

Lastly, this survey elicited subjective perceptions of eyewitnesses of multiple vs. single perpetrator crimes. Notably, half of respondents viewed eyewitnesses of multiple perpetrator crimes to be "as good as" witnesses of single perpetrator crimes. This was unexpected in light of the fact that research demonstrates that viewing multiple perpetrators consistently reduces identification and recognition performance (Bindemann, Sanford, Gillat, Avetisyan, \& Megreya, 2012; Clifford \& Hollin, 1981; Megreya \& Burton, 2006). Yet in the lab, researchers may manipulate the number of perpetrators while controlling for all other encoding and retention variables. By contrast, an eyewitness called in to view a lineup may have encountered any of the myriad of variables known to influence eyewitness identification accuracy. It may be that police do not experience this difference consistently because they encounter eyewitnesses of single perpetrator crimes whose memory is equivalently impacted by other variables (Krix, Sauerland, Lorei, \& Rispens, 2015). This is an area where police perceptions do not map onto what we might expect based on data and theory. Although we cannot explain why based on these data, it is an area of divergence between police perception and science.

This survey provides an initial understanding of police practice that can inform experimental research. For example, researchers may want to use an ecologically valid control group against which to test novel identification procedures adapted specifically to support eyewitnesses of multiple perpetrator crimes. In this case, it is useful to consider both the consistent use of sequential, photographic lineups, and the inconsistent practice of separating suspects into separate lineups. Meanwhile, this survey also serves to highlight one area in which there is clearly need for more research in the context of multiple perpetrator recognition and identification: contextual memory cues. The extent to which different contextual cues enhance or undermine memory for multiple perpetrators is particularly relevant when considering whether or not to (1) indicate the alleged role of a suspect before the identification decisions, (2) delay lineup presentation until all suspects are available, and (3) present multiple suspects in the same lineup.

\section{LIMITATIONS}

While the current research reveals interesting findings in police perceptions and identification practice in the context of multiple perpetrator crimes, it is important to note 
that some limitations constrain the generalizability of these findings. Limitations in sample size and sample selection means this is by no means a comprehensive overview of identification procedures for each country. The initial sample size of 51 officers was reduced to 33 officers by the end of the survey. Also, because one police contact from each country was used to recruit police responses, it is plausible there is selection bias in our data. It is therefore not our intent to claim that these results are representative of how each country as a whole conducts identification procedures for multiple suspects. However, we do consider this an initial collection of examples of how some police officers within these countries report conducting identification procedures with multiple suspects. This provides us with valuable information for future research designs and a context in which to place results on multiple perpetrator recognition and identification.

\section{CONCLUSIONS}

The current research presents results from a survey of police officers in three European countries (Sweden, Belgium, and the Netherlands) on experiences conducting identification procedures for cases involving multiple perpetrators. Guidelines provided to officers on the topic of multiple perpetrator identification were scarce, if available at all. While officers in these countries were consistent in reporting the use of sequential photographic lineups and eliciting role assignment following an identification decision, they also varied in reports of procedures such as separating vs. grouping suspects in lineups, whether the same or multiple police officers are in charge of lineup construction, and whether to present lineups as suspects become available vs. when all suspects are available. Future research should explore whether procedures that provide context are useful to enhance eyewitness identification accuracy for multiple perpetrators. 


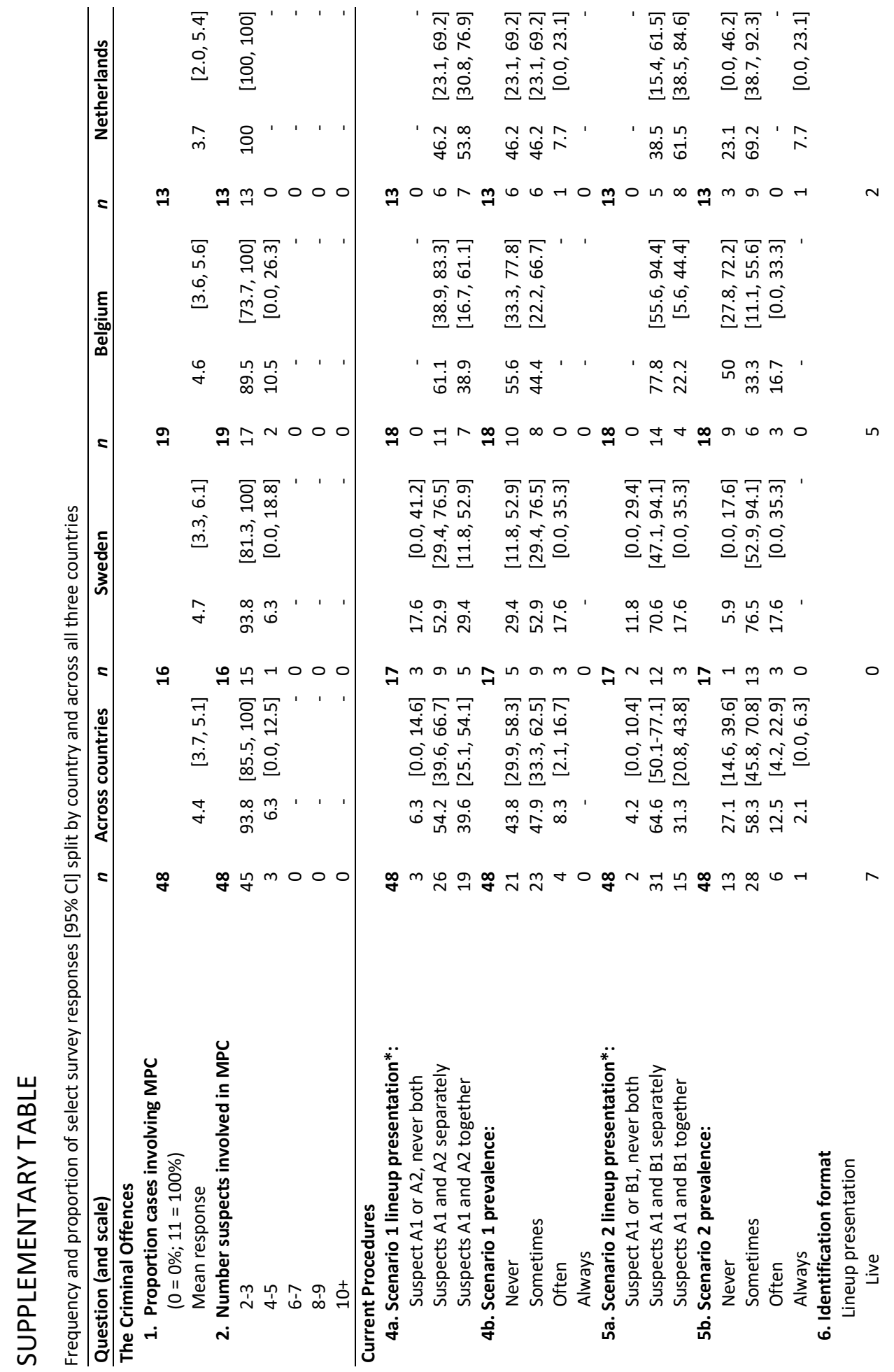




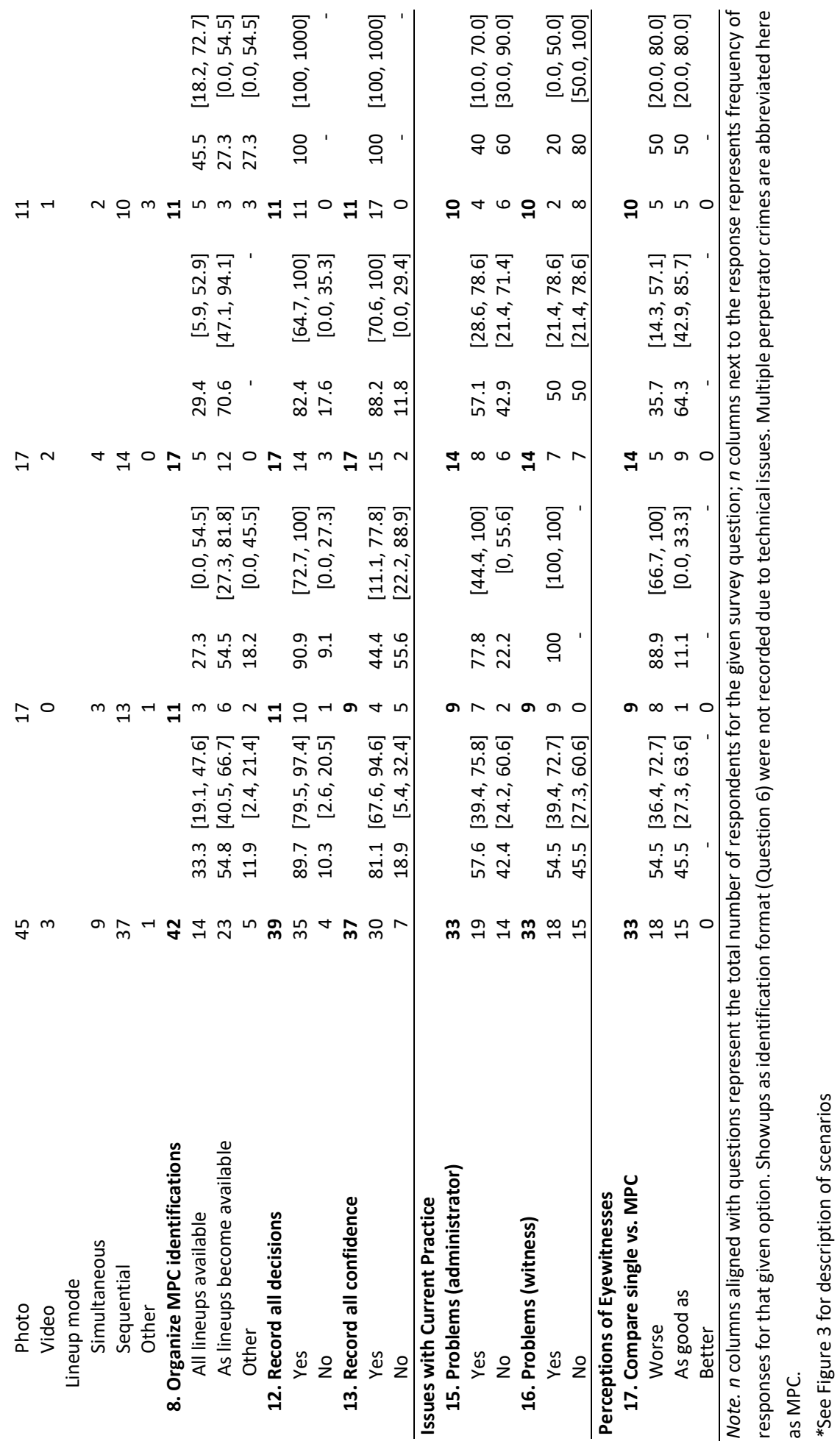


CHAPTER 3 
CHAPTER 3

\section{Showup identification decisions for multiple perpetrator crimes: Testing for sequential dependencies}

Tupper, N., Sauerland, M., Sauer, J., Broers, N., Charman, S., \& Hope, L. (2018). Eyewitness identification for multi-perpetrator crimes: Testing for sequential effects in multiple showup decisions. Manuscript submitted for publication, revision invited. 


\begin{abstract}
Research in perception and recognition demonstrates that a current decision ( $i$ ) can be influenced by previous ones $(i-j)$, meaning that subsequent responses are not always independent. Experiments 1 and 2 tested whether initial showup identification decisions impact choosing behavior for subsequent showup identification responses. Participants watched a mock-crime film involving three perpetrators and later made three showup identification decisions, one showup for each perpetrator. Across both experiments, evidence for sequential dependencies for choosing behavior was inconsistent. In Experiment 1, responses on the third, target-present showup assimilated towards previous choosing. Yet, in Experiment 2, responses on the second showup contrasted previous choosing regardless of target-presence. Experiment 3 examined whether differences in stimuli and number of test trials in the eyewitness (vs. basic recognition) paradigm could account for the absence of hypothesized patterns of sequential dependencies in Experiments 1 and 2. Sequential dependencies were detected in recognition decisions over many trials, including recognition for faces: the probability of a yes response on the current trial increased if the previous response was also yes (vs. no). However, choosing behavior on previous trials did not predict individual recognition decisions on the current trial. Thus, while sequential dependencies did arise to some extent, results suggest that the integrity of identification and recognition decisions are not likely to be impacted by making multiple decisions in a row.
\end{abstract}


In October 2015, news outlets (Frontière Belge, 2015) featured security footage of an unresolved case: the attempted abduction of a truck driver on the French-Belgian border. As the truck driver walked around the rear of his truck, two men appeared and attacked him. While the two perpetrators struggled to force the driver into the back of a waiting car, an elderly passerby intervened, pulling at the perpetrators' jackets and trying to place himself between them and the truck driver. Following the failed abduction and a hurried, but equally unfruitful search for the truck driver's keys, the two men fled the scene by car.

This case is just one example of the many violent crimes that are committed by multiple perpetrators. Gang violence (Juodis, Woodworth, Porter, \& Ten Brinke, 2009), hate crimes (Sandholtz, Langton, \& Planty, 2013), rapes (Horvath \& Kelly, 2009), and assaults (Hobson, Wilcock, \& Valentine, 2012) are often committed by perpetrators working together. In fact, the rising rate of such crimes appears to be a global phenomenon. For example, in Finland, Sweden, and the Netherlands, 13-17\% of homicides between 2003 and 2006 involved two or more perpetrators (Liem et al., 2013) while the proportion of homicides with multiple perpetrators in the U.S. reached $20 \%$ in 2008 (nearly double that reported in 1980; Cooper \& Smith, 2011). These crimes often involve victims or bystanders as eyewitnesses-like the driver and the passerby above-who may be asked to identify multiple suspects related to the multiple perpetrators. Yet, the decades of research focused on uncovering and understanding factors that affect accuracy in eyewitness identification procedures typically focuses only on identifications of a single perpetrator, providing little empirical evidence to support or oppose recommendations in protocols specific to the context of multiple perpetrator crime. Should police departments, for instance, follow the example of the U.K. and multiply "best practice" by creating a new lineup for each suspect of a different perpetrator (Police and Criminal Evidence Act [PACE], 1984)? If so, does the order of presentation of identification tests affect the reliability of the evidence obtained? Or does the act of making multiple identification decisions affect the decisions themselves?

In this paper, we address this last question, examining the consequences of testing memory for multiple perpetrators (Malmberg, Lehman, Annis, Criss, \& Shiffrin, 2014). We present three experiments examining whether current showup identification decisions are associated with witness choosing behavior on previous showup decisions. We aimed to determine whether sequential dependencies (i.e., whether choosing behavior on previous tests influences choosing on a current test) should be considered in cases when eyewitnesses are asked to make multiple identification decisions, specifically when those decisions pertain to the different suspects in a multiple perpetrator crime. 


\section{IDENTIFICATION OF MULTIPLE PERPETRATORS}

Clifford and Hollin (1981) first revealed the difficulty of eyewitness identification in the context of multiple perpetrator crimes when they had participants view a non-violent event with one, three, or five perpetrators. Despite only having to select the main perpetrator from a target-present lineup immediately following the crime, only $30 \%$ of participants in the three-perpetrator condition and $20 \%$ in the five-perpetrator condition made accurate identifications (compared with $40 \%$ in the one-perpetrator condition). More recently, Megreya and Bindemann (2012) demonstrated a similar drop in accuracy with as few as two unfamiliar faces to be encoded. Participants viewed a mock crime with one perpetrator alone or with an accomplice and were subsequently asked to identify the perpetrator. The presence of a second person at encoding was associated with decreased identification accuracy in target-present lineups (lower hit rates and higher miss rates). Approximately $54 \%$ of participants who saw the perpetrator alone were able to accurately identify him/her, compared with only $29 \%$ of participants who saw the perpetrator with an accomplice.

To date, three procedures have been proposed to address the applied issue of the multiple perpetrator identification disadvantage. The two-person serial lineup (Wells \& Pozzulo, 2006), the elimination lineup (Dempsey \& Pozzulo, 2008; 2013), and an adapted sequential identification procedure (Hobson \& Wilcock, 2011) were each tested against traditional simultaneous lineups, sequential lineups, or both. The results were mixed, and any improvements associated with these methods depended upon which target identity was being presented (i.e., accomplice vs. perpetrator), the presence or absence of the target in the lineups, or both. Unfortunately, when these new methods fall short, we do not know if it is because the proposed adaptations did not address the mechanisms they intended to, or if the theories used to justify these adaptations are ultimately not relevant to the multiple perpetrator identification disadvantage. For example, the two-person serial lineup is intended to provide context to aid memory by presenting the sequential lineups of the culprit and of the accomplice at the same time (Wells \& Pozzulo, 2006). While the lineups for each are flashed side-by-side on the screen, the two suspects are never shown simultaneously, but always paired with a filler. In theory, the context of one face should aid our ability to recognize or reject the other face. But when this lineup does not improve identification accuracy, is it because contextual cuing is not useful for faces in a lineup context? Or is it because the suspects are never shown together, and thus are not cuing memory? It is difficult to interpret these results because they are premature attempts to fix problems that are still not well understood, meaning the adapted lineups amount to trialand-error solutions. 
Shallow encoding (Megreya \& Burton, 2006) and increased memorial demand (Bindeman, Sanford, Gillatt, Avetisyan, \& Megreya, 2012) have more recently been explored as reasons for the decreased identification performance for multiple perpetrator crimes, and both appear to play a role. However, there is another independent factor that is unique to multiple perpetrator identification that has yet to be considered: the decisional structure of making multiple identifications. Below, we explore how the act of making multiple identifications may undermine the integrity of those decisions.

\section{SEQUENTIAL DEPENDENCIES IN PERCEPTION AND RECOGNITION}

An individual police lineup has been likened by researchers to a real-world signal detection decision, but with the modification to include filler (i.e., non-suspect) misidentifications (Palmer, Brew, \& Weber, 2010; 2012). The signal detection model, however, mathematically assumes independence of trials, for which a decision is based solely on the evidence present in that trial. In contrast, research in perception and memory demonstrates that a current decision ( $i$ ) can be influenced by a previous one $(i-j)$, so that a current response may favor (assimilation) or disfavor (contrast) the preceding responses (Treisman \& Williams, 1984). In other words, in a series of trials presented one-afteranother, the responses, although separate, are not independent. These sequential dependencies appear in perception, classification, and recognition tasks where participants make multiple, sequential decisions (see Malmberg \& Annis, 2012 for a review) -tasks that present a theoretical overlap with making multiple eyewitness identification decisions.

Sequential dependency can be demonstrated in its simplest form in a traditional detection experiment. Howarth and Bulmer (1956) seated participants in a dark room with a flash-bulb set at a 50\% detection rate at a given intensity, meaning that the light was bright enough to be detected, but dim enough that participants only reported seeing it half of the time. The momentary flashes were accompanied by the sound of a bell, so that when participants heard the bell ring, they indicated whether or not they had seen the flash of light (yes vs. no). At 50\% detection, participants will make errors half of the time; errors that should theoretically display natural fluctuations and therefore appear randomly throughout the hundreds of trials. However, participants demonstrated a tendency to assimilate responses towards previous ones, meaning that a no response was more likely to be followed by another no response than a yes response. Further still, at some points, the light signal was omitted so that the bell rang without the accompanying light flash. When the experimenters forced a sequence of three of these blank trials (no-no-no), they found the same degree of assimilation for the subsequent fourth response as for three natural occurring negative responses. Such sequential dependencies are found in a variety of tasks, 
including absolute judgments of sound (Holland \& Lockhead, 1968) and the perceptual classification of facial expressions (Hsu \&Yang, 2013).

The mechanism underlying sequential dependencies remains a subject of ongoing debate, with attempts to model sequential dependencies favoring one of the two systems involved in a perception task: the decisional processes and the cognitive system. Some models consider sequential dependencies to arise from biased decision-making (Treisman \& Williams, 1984). According to these models, assimilation results from the observer's short-term assumption that the most recent stimulus is also the most likely to occur again. However, patterns of contrasting answers are the result of the observer attempting to correct decisional criteria to a desirable level in the long-term. These fluctuations in response bias purport to explain why judgments show assimilation immediately following trial $i$, but revert to contrast after a few trials. On the other side of the debate are models arguing that sequential dependencies arise either entirely, or at least in part, from the cognitive system (Brown, Marley, Donkin, \& Heathcote, 2008; Matthews \& Stewart, 2009; Stewart, Brown, \& Chater, 2005). In these models, sequential dependencies arise as a result of inappropriate information being carried forward from the previous trial, affecting the perception of the current stimulus.

Malmberg and Annis (2012) were the first to demonstrate sequential dependencies in recognition memory. They presented a series of experiments using traditional recognition paradigms and judgments of frequency recognition tasks to approximate the perception and categorization tasks that routinely demonstrate sequential dependencies. For example, in one experiment, participants studied 40 word pairs and were later tested on their recognition for those words among never-studied words. As with Howarth and Bulmer's (1956) light-detection experiment, participants were more likely to respond old if they had responded old (rather than new) on the previous trial, regardless of whether the previous response was correct (hit) or incorrect (false alarm). The appearance of sequential dependencies was consistent across several replications with different stimuli, including landscape images, and picture-word pairs (Malmberg \& Annis, 2012).

\section{THE CURRENT RESEARCH}

Studies investigating the cause of the multi-face recognition disadvantage (Megreya \& Burton, 2006; Bindemann et al., 2012) tend to focus on the encoding conditions: how factors that affect perception and attention interfere with encoding, and thus damage chances of identification from the outset. Consequently, studies adapting lineups that were originally designed for single-perpetrator crimes so far considered these encoding difficulties and adjusted methodology in attempts to compensate for the resulting impoverished memory (Wells \& Pozzulo, 2006; Hobson \& Wilcock, 2011). While this is a 
reasonable starting point to investigate multiple perpetrator identifications, it is also important to explore other factors that may affect identification decisions. In this vein, we investigated the possibility of sequential dependencies within the eyewitness paradigm. Specifically, how does the act of making multiple identification decisions for unique perpetrators affect the validity of those decisions? Despite the clear theoretical relevance of sequential dependencies to witnesses making identification decisions for multiple perpetrators, the phenomenon remains untested in the eyewitness identification context.

Multiple perpetrator crimes present a framework in which relatively few sequential decisions are made, and in which these decisions have serious consequences. Sequential dependencies measured in the recognition paradigm have little substantial effect on overall recognition accuracy because the beneficial and detrimental sequences of dependencies will typically balance out over the many trials, reducing its impact on the overall accuracy for recognition (Malmberg \& Annis, 2012). Considering identification paradigms lack the many trials needed to balance out recognition accuracy, the appearance of sequential dependencies in this context would be a matter of substantial impact and cause for concern. Therefore, we tested for sequential dependency effects within the eyewitness identification context by having participants make multiple, consecutive showup decisions.

Show ups are particularly well-suited for an initial test for sequential dependencies within the eyewitness context for three reasons. First, showups (live or photographic) are a common identification procedure around the world (Davis, Valentine, Memon, \& Roberts, 2015; Police Executive Research Forum, 2013). Second, forced-report showup decisions (/s this the perpetrator? Yes vs. no) emulate the binary-decision tasks in which sequential dependencies have been consistently observed. Third, showups permit a controlled investigation of sequential dependencies on identification decision-making free from the influence of lineup construction variables (e.g., filler similarity, lineup presentation method). If sequential dependencies are found to affect showup decision-making, subsequent investigations can determine how these effects interact with lineup composition and presentation variables.

Across two initial experiments, we examined the relation of previous identification decisions to subsequent choosing behavior in the context of the multiple showup identification decisions for a multiple perpetrator crime. If it is possible to predict current choosing on a showup identification decision from previous choosing, it provides initial evidence that sequential effects may be present in multiple showup identification decisions. Given that research has previously demonstrated that sequential dependencies in recognition are a result of interference from previous trials (Malmberg \& Annis, 2012), Experiments 1 and 2 considered both previous signal (target-presence: present vs. absent) 
and previous response (Choosing: yes vs. no) as predictors of current choosing behavior (Matthews \& Stewart, 2009). We expected that initial showup responses would predict choosing for subsequent showup responses. In other words, choosing on a previous showup identification would be associated with choosing on subsequent ones, and rejecting on a previous showup identification would be associated with rejecting on subsequent ones. We also expected previous target-presence to be separately associated with the current identification decision, such that when the previous target being present would predict current choosing and the previous target being absent would predict current rejecting (Matthews \& Stewart, 2009).

We also considered the possibility of an interaction between current targetpresence and previous choosing on the current choosing, such as being confronted with a target-absent trial would further raise the probability of rejection given a previous rejection. Non-memorial factors tend to exert stronger effects on recognition memory tasks when the target stimulus is absent and there is no opportunity for genuine recognition (Palmer, Sauer, \& Holt, 2017). In other words, if memory is not able to provide the answer, people look for other cues to influence their decision. In this way, sequential dependencies might represent an attempt to use imperfect cues to guide decision-making under conditions of uncertainty (Gigerenzer, Hoffrage, \& Kleinboelting, 1991). Straightforward sequential dependencies should arise regardless of target presence, however, it is possible that the strength of the effect will vary depending on whether the target is present or not.

Although Experiments 1 and 2 were conducted separately, they used similar methodologies and analyses to answer the same question. Thus, the data are not collapsed across experiments, but the methods and results are presented together.

\section{EXPERIMENTS 1 AND 2}

\section{ETHICS STATEMENT}

These studies were approved by the ethical review board of the Faculty of Psychology and Neuroscience of Maastricht University (ECP-157_01_10_2009_A1). Written consent was obtained from participants in Experiment 1. Participants in Experiment 2 provided consent by clicking the button to continue the experiment.

\section{PARTICIPANTS AND DESIGN}

A total of 411 participants were tested, 404 of which were included in analyses. Participants either completed the experiment in the lab (Experiment $1, N=120$ ) or online (Experiment 2, $N=291$ ). The average age of participants was 21 years $(M=20.77, S D=3.64)$. They were compensated with a $€ 5$ gift voucher (Experiment 1 ) or participation credit (Experiments 1 and 2). 
Participants viewed a three-person mock crime video and were subsequently presented with three photographic showups, one for each of the three perpetrators. In Experiment 1, we aimed to provide an initial test of sequential dependency in facial identification. Four conditions were chosen to optimize conditions for sequential dependencies through an established pattern of target-present and target-absent showup photographs (Howarth \& Bulmer, 1956). The first and second showups were always consistent in target-presence; they were either both target-absent (TA) or both targetpresent (TP), while the third showup was either consistent or different, leading to four conditions with targets: (1) TA/TA/TP, (2) TA/TA/TA, (3) TP/TP/TA, and (4) TP/TP/TP. In retrospect, we realized this also meant that we were not able to disentangle the effect of target-presence between showups 1 and 2 on showup 2. Thus, Experiment 2 implemented all combinations of target presence by adding four additional conditions with targets: (5) TA/TP/TA, (6) TA/TP/TA, (7) TP/TA/TP, and (8) TP/TA/TA. Presentation order of targets (i.e., $123,132,231,213,312,321)$ was counterbalanced for both experiments.

\section{GENERAL METHOD FOR EXPERIMENTS 1 AND 2}

\section{Materials.}

Crime video. In the 2:45 min mock crime video, the male victim arrives by bike and locks it against a railing with other bikes. Three target people, one woman and two men, are shown in the background gesturing towards the victim. When the victim walks into a nearby building, the thieves use a hand-saw to break the locks of two bikes, including the victim's, and walk away with the bikes. Each target actor in the video has approximately $15-$ $20 \mathrm{~s}$ of close-up shots in which their faces are clearly visible.

Targets. All three targets were Caucasian university students. The female target has an average build, long, straight, blond hair and was judged by pilot participants to be approximately 22 years old. The first male target has an average build with short, dark-blond hair, and slight facial hair, and was judged to be approximately 25 years old. The second male target was comparatively shorter to the first target, with short, blond hair, and was judged to be approximately 23 years old.

Showups. Three target-absent and three target-present showups were constructed, one for each of the three perpetrators. The showups consisted of color photographs $4.39 \times 5.89 \mathrm{~cm}$ in size. The targets were photographed on the same day as the stimulus event was filmed, but wore different clothing. One innocent suspect was selected as a replacement for each target in the target-absent showups. The replacements were chosen based on similarity to the actual target, as established by a pilot study with $N=22$ participants (age: $M=27.45, S D=12.14$ ). Specifically, replacements were rated as statistically similar to the perpetrator with regard to memorability, distinctiveness, and typicality (Wickham, Morris, \& Fritz, 2000; Vokey \& Read, 1992). Participants were also 
asked to judge the similarity of the target faces paired with each of their possible replacements. This comparison score was used to match for similarity across the three target-replacement pairings, so that each of the three target-absent showups would be equally difficult for participants to judge. Results of the pilot study are available in the appendix (Table A3.1).

\section{PROCEDURE}

Participants arrived at the lab for individual testing sessions (Experiment 1 ) or received a Qualtrics (Qualtrics, 2016) link to complete the experiment online (Experiment 2). Participants were informed that the experiment would be administered using a selfpaced computer task. After giving informed consent, participants were told that they would be shown a video and were instructed to pay close attention as they would be asked questions about it later. After watching the mock crime video, participants completed a 20 30 min filler task by answering a series of questionnaires (Experiment 1 and 2) or by completing a combination of search tasks and word-generation games (Experiment 2). Next, participants were reminded that they had seen a film of three thieves stealing a bike, and were now considered eyewitnesses. They were instructed: You will be shown a series of three photographs. Each photograph is one suspect for each of the three bike thieves. For each photograph, please decide whether or not the person shown was one of the perpetrators. Once you make a decision, you will move on to the next photo. A subsequent screen displayed a one-time warning that the persons in the photographs may or may not be the actual perpetrators.

Participants were then shown a photo for one of the perpetrators (Suspect 1: Present or absent). A forced-report question asked if the person shown was one of the perpetrators (yes or no), after which they were asked to indicate how certain they were in their decision (0-100\%). The procedure was repeated for Suspects 2 and 3 . Although suspects are numbered here for convenience, presentation order of targets was counterbalanced; meaning Suspect 1 for the eyewitness could correspond to any of the three perpetrators. Following all identification decisions and confidence ratings, participants were shown the photos of those they had positively identified and asked to name the role each played in the crime. However, role assignment and confidence are outside of the scope of the current research and are therefore not addressed further. Finally, participants were thanked for their time and debriefed.

Experiment 2 differs from Experiment 1 in two ways. In order to determine whether participants had watched the entire video, a still image of a white arrow and the text "This is a white arrow, please remember this arrow as you will be asked about it later" was added for the last $7 \mathrm{~s}$ of the video (after the target event). Following the filler task, participants were asked to name the shape and color of the shape presented at the end of 
the video. This section of the computer task was timed so that the task advanced automatically after 2:52 min regardless of whether or not the video was paused. Therefore, participants who could not correctly name the shape and color $(n=4)$ were assumed to have not completed the video and were removed from all analyses. A final question prompted participants to describe the environment in which they completed the experiment (e.g., time of day, location, presence of others).

\section{RESULTS}

In Experiment 1, all 120 participants were retained for data analysis. In Experiment 2 , seven participants were removed from data analysis for answering the control question incorrectly (4), not completing the filler task (2), or because Qualtrics recorded their experiment duration time as exceeding four hours and the participant did not respond to requests to elaborate (1), leaving 284 participants.

Descriptive statistics for choosing on showups. Across the three showup identification decisions in both Experiments 1 and 2, choosing rates were low, at 34-42\%. Overall, only $4-12 \%$ of participants chose on all three showups. Meanwhile, $15-22 \%$ of participants rejected all decisions. Less than half of participants (26-47\%) chose on at least two showups. See Table 3.1 for choosing rates for each experiment.

Table 3.1

Experiments 1 and 2: Proportion (Frequency) of Choosing Across Showups and Overall

\begin{tabular}{|c|c|c|c|c|c|c|c|}
\hline & \multicolumn{3}{|c|}{ Choosing by showup } & \multicolumn{4}{|c|}{ Overall Choosing } \\
\hline & Showup 1 & Showup 2 & Showup 3 & 0 chosen & 1 chosen & 2 chosen & 3 chosen \\
\hline Expt. 1 & & & & $.15(9)$ & $.38(23)$ & $.35(21)$ & $.12(7)$ \\
\hline $\mathrm{TP}$ & $.53(32)$ & $.53(32)$ & $.52(31)$ & & & & \\
\hline TA & $.25(15)$ & $.23(14)$ & $.27(16)$ & & & & \\
\hline Overall & $.39(47)$ & $.38(46)$ & $.39(47)$ & & & & \\
\hline Expt. 2 & & & & $.22(62)$ & $.43(120)$ & $.30(84)$ & $.04(12)$ \\
\hline TP & $.54(76)$ & $.43(62)$ & $.58(82)$ & & & & \\
\hline TA & $.25(36)$ & $.25(35)$ & $.29(39)$ & & & & \\
\hline Overall & $.41(114)$ & .34 (93) & .42 (117) & & & & \\
\hline
\end{tabular}

Note: Displayed under "Choosing by showup" are proportions of participants choosing on target-present and target-absent showups. Displayed under "Overall Choosing" are proportions of participants who chose on zero, one, two, or three showups. Raw frequencies are between parentheses. TA denotes target-absent showups and TP denotes target-present showups

Experiment 1: Testing for sequential dependencies. In order to establish the association of previous identification decisions and both previous and current targetpresence with current identification decisions, we performed separate binary logistic regressions for choosing on the second and third showup. For example, for choosing on the second showup, we entered previous target-presence (absent vs. present on Showup 1), current target-presence (absent vs. present on Showup 2) and previous choosing (yes vs. no 
on Showup 1) as predictors. For choosing on the third showup, we used previous targetpresence, current target-presence (Showup 3), and previous choosing (yes vs. no on Showup 1 and Showup 2) as predictors. Because target-presence for the first and second showups did not vary in Experiment 1, target-presence for Showups 1 and 2 were included as a single predictor.

In the initial analyses for Showup 2, we included all main effects in the equation. In the initial analyses for Showup 3, we included all main effects and the current targetpresence by previous response (selection vs. rejection) interaction. We then sequentially excluded the interaction if non-significant and any non-significant main effects by order of distance from the current decision. However, given our theoretical predictions, previous choosing was always included in the final model. Although we present the results descriptively here, relevant statistics can be found in Table 3.2.

Choosing behavior on the second showup. Only target-presence was a significant predictor in the final model. Participants were more likely to choose when the target was present. However, due to the fact that target-presence for Showups 1 and 2 did not vary within subjects, it is unclear if it is current target-presence, previous target-presence, or both that are associated with choosing behavior for Showup 2.

Choosing behavior on the third showup. The current target-presence by previous choosing interaction was significant. Examination of simple effects revealed that only when the current trial was target-present, choosing on Showup 2 predicted choosing on Showup 3 such that participants who chose on the second showup were 5.88 times more likely to also choose on the third compared with participants who had rejected the second showup. In other words, 79\% of those who chose on Showup 2 also chose on a target-present Showup 3, while only $39 \%$ of those who rejected Showup 2 subsequently chose on a targetpresent Showup 3.

Experiment 2: Testing for sequential dependencies. Analyses for Experiment 2 were analogous to Experiment 1 with the exception that all initial models included the current target-presence by previous response interaction. See Table 3.2 for the relevant statistics. 
Table 3.2

Experiments 1 and 2: Models of Logistic Regressions Predicting Choosing on Showups 2 and 3 Based on Previous Choosing and Target-Presence

\begin{tabular}{|c|c|c|c|c|c|c|c|}
\hline & \multirow[b]{2}{*}{$b$} & \multirow[b]{2}{*}{$S E$} & \multirow[b]{2}{*}{ Wald } & \multirow[b]{2}{*}{$p$} & \multicolumn{3}{|c|}{ 95\% Cl for Odds Ratio } \\
\hline & & & & & Lower & Odds & Upper \\
\hline \multicolumn{8}{|l|}{ Experiment 1} \\
\hline \multicolumn{8}{|l|}{ Showup 2} \\
\hline Choosing 1 & -0.10 & 0.42 & 0.06 & .813 & 0.40 & 0.91 & 2.06 \\
\hline Target-Presence 1 and 2 & 1.35 & 0.42 & 10.41 & .001 & 1.70 & 3.87 & 8.79 \\
\hline Constant & -1.17 & 0.32 & 13.14 & $<.001$ & & 0.31 & \\
\hline \multicolumn{8}{|l|}{ Showup 3} \\
\hline Choosing 2 & -0.45 & 0.60 & 0.57 & .451 & 0.20 & 0.64 & 2.07 \\
\hline Choosing 1 & 0.23 & 0.42 & 0.32 & .573 & 0.56 & 1.26 & 2.85 \\
\hline Target-Presence 3 & 0.36 & 0.50 & 0.53 & .469 & 0.54 & 1.44 & 3.81 \\
\hline Choosing $2 \times$ TP 3 & 2.23 & 0.89 & 6.31 & .012 & 1.63 & 9.27 & 52.62 \\
\hline Constant & -0.91 & 0.40 & 5.08 & .024 & & 0.40 & \\
\hline \multicolumn{8}{|l|}{ Showup 3 , reversed ${ }^{a}$} \\
\hline Choosing 2 & 1.77 & 0.65 & 7.47 & .006 & 1.65 & 5.88 & 20.96 \\
\hline Choosing 1 & 0.23 & 0.42 & 0.32 & .573 & 0.56 & 1.26 & 2.85 \\
\hline Target-Presence, reversed & -0.36 & 0.50 & 0.53 & .469 & 0.26 & 0.70 & 1.85 \\
\hline Choosing $2 \times$ TP 3 & -2.23 & 0.89 & 6.31 & .012 & 0.02 & 0.12 & 0.61 \\
\hline Constant & -0.55 & 0.37 & 3.21 & .137 & & 0.58 & \\
\hline \multicolumn{8}{|l|}{ Experiment 2} \\
\hline \multicolumn{8}{|l|}{ Showup 2} \\
\hline Choosing 1 & -0.54 & 0.27 & 4.04 & .044 & 0.34 & 0.58 & 0.99 \\
\hline Target-Presence 2 & 0.87 & 0.26 & 11.22 & .001 & 1.44 & 2.39 & 3.40 \\
\hline Constant & -0.92 & 0.21 & 18.70 & $<.001$ & & 0.40 & \\
\hline \multicolumn{8}{|l|}{ Showup 3} \\
\hline Choosing 2 & 0.40 & 0.27 & 2.27 & .132 & 0.89 & 1.50 & 2.52 \\
\hline Choosing 1 & -0.17 & 0.26 & 0.43 & .512 & 0.50 & 0.84 & 1.41 \\
\hline Target-Presence 3 & 1.28 & 0.26 & 24.90 & $<.001$ & 2.17 & 3.59 & 5.93 \\
\hline Constant & -1.04 & 0.23 & 20.22 & $<.001$ & & 0.35 & \\
\hline
\end{tabular}

Note: Variables were coded as follows. Choosing: non-choosing $=0$, choosing $=1 ;$ target-presence: $\mathrm{TA}=0, \mathrm{TP}=1$. Experiment 1. Showup 2: $R^{2}=.09$ (Cox \& Snell), .13 (Nagelkerke). Model $\chi^{2}(2)=11.71, p=.003 ;$ Showup 3: $R^{2}=.14$ (Cox \& Snell), .18 (Nagelkerke). Model $\chi^{2}(3)=17.50, p=.002$. In order to examine the target-presence by previous choosing interaction, the variable TP 3 was reverse-coded so that TA $=1, T P=0$. aShowup 3 , reversed represents the regression that was conducted using the reverse-coded target-presence variable and reported in results. Experiment 2. Showup 2: $R^{2}=.05$ (Cox \& Snell), .07 (Nagelkerke). Model $\chi^{2}(2)=15.10, p=.001 ;$ Showup 3: $R^{2}=.10$ (Cox \& Snell), .14 (Nagelkerke). Model $\chi^{2}(3)=30.07, p<.001 . \mathrm{Cl}=$ Confidence Interval.

Choosing behavior on the second showup. As expected, choosing on Showup 1 was a significant predictor of choosing on Showup 2. However, current choosing contrasted previous choosing, so that participants were 1.72 times less likely to choose on the second showup if they had chosen on the previous one. In other words, $72 \%$ who chose on Showup 1 subsequently rejected Showup 2. Meanwhile 62\% of participants who rejected Showup 1 went on to reject Showup 2. The lack of significant interaction for current target-presence by previous choosing indicates that this sequential dependency was not affected by the 
current presence of the target. However, current target-presence was also a significant predictor for choosing.

Choosing behavior on the third showup. For choosing on Showup 3, only current target-presence was a significant predictor.

\section{DISCUSSION}

Experiments 1 and 2 were initial tests for sequential dependencies across multiple showup identification decisions in the context of multiple perpetrator crimes. We expected previous responses (choosing) and previous target-presence to be related to current decisions. While we did find some evidence for sequential dependencies in both experiments, effects were not consistent. In Experiment 1, when the current trial was target-present, participants who chose on the second showup were nearly six times more likely to also choose on the third showup compared with those who had rejected the second showup (assimilation). Although we did expect to find an interaction between current target-presence and previous choosing, the interaction operated counter to expectations. By contrast, in Experiment 2, participants who chose on the first showup, were more than twice as likely to not choose on the second trial (contrast), regardless of target-presence. Taken together, results from both Experiments 1 and 2 provide inconsistent evidence for the expected sequential dependencies. This inconsistency is surprising given the theoretical overlap to fields that have robustly produced sequential dependencies, including perception, absolute identification, and, most pertinently, recognition.

In recognition tests, Malmberg and Annis (2012) found sequential dependencies between previous and current responses: $A$ hit on a previous trial increased the probability of a hit on a current trial, but previous hits and false alarms also increased the probability of false alarms on a current trial. In essence, participants were more likely to choose on a current trial if they had chosen on a previous one. This effect was replicated with a variety of paired stimuli (e.g., landscape photo pairs, non-word pairs), as well as with a single-item classic recognition test. While the current research retains similarities to these basic recognition paradigms, as well as other contexts in which sequential dependencies have robustly appeared (i.e., perception, categorization tasks (Holland \& Lockhead, 1968; Hsu \& Yang, 2013), the eyewitness paradigm also presents differences that may explain the inconsistent results reported here. Consequently, we considered potentially important differences that may explain the inconsistent results reported here. First, our experiments focused on the recognition of faces, rather than images, words, or non-words. While sequential dependencies have been found in categorization for facial expression (Hsu \& Yang, 2013), for example, they have not been tested specifically for face recognition. Some evidence in perceptual and recognition research gives us reason to suspect that individuals 
process and remember faces differently than other non-face images (e.g., detecting minute changes in facial features; Diamond \& Carey, 1986; capacity limits in face processing; Bindemann, Burton, \& Jenkins, 2005; Bindemann, Jenkins, \& Burton, 2007). Given that sequential dependencies in recognition are thought to arise from mnemonic or perceptual processing, such differences in stimuli may be important.

Second, the number of stimuli in our experiment differs greatly from a basic recognition paradigm. In a typical recognition experiment, participants are presented with long lists of words or images, given little time to study these items, and are then tested on those items along with never-before-seen items. Conversely, our experiment only included three perpetrators to study over the course of a 2.5 min mock crime video. Although we cannot ignore the possibility that there are simply not enough stimuli being studied, and therefore participants are not uncertain enough to rely on previous responses, the maximum average participant accuracy rates of $65 \%$ do suggest that our filler task allowed for sufficient memory decay to induce uncertainty. Meanwhile, sequential dependencies in recognition are thought to be a result of interference from previous trials that affect mnemonic processing during testing. Therefore, it seems more likely that our results reveal a difference during testing rather than a difference during encoding.

A third difference lies in the number of trials during the testing phases. While recognition experiments may have tens or hundreds of test trials, our participants encountered only three. Perhaps this is not a sufficient number of trials for sequential dependencies to arise. Sequential dependencies have been explained through accumulator models, which predict shifts over time based on criterion placement or accumulation starting points (e.g., Selective Attention, Mapping and Ballistic Accumulation [SAMBA]; Brown et al., 2008; Matthews \& Stewart, 2009). The SAMBA model, for example, posits that a participant classifying the loudness of a sound (i.e., soft vs. loud; Jones, Love, \& Maddox, 2006) uses the sound on initial trials to generate a range between which the subsequent sounds are expected to fall. This range establishes how soft the participants can expect a soft sound to be and how loud they can expect a loud sound to be. When confronted with the task of classifying the sound on the current trial, the observer will compare the sound to the upper and lower range in relation to the loudness of the previous response. Their response will depend upon the strength of the evidence for each of these answers. When a soft response is given on the current trial, it is hypothesized that this biases the perception of the sound on the subsequent trial by temporarily reducing the strength of evidence needed to favor another soft response. Thus, assimilation arises from the decision making: because the soft response now has the advantage, the following trial is more likely to reach the threshold to be classified as soft. Contrast, however, arises from the perceptual mechanisms: because observers are comparing the current sound to the previous one, any 
change louder or softer can lead to over- and underestimation of the strength of that sound. In this model, assimilation and contrast both occur because the stronger effect (assimilation) eventually decays to give way to the weaker one (contrast; Brown et al., 2008). It is possible that such models require an adjustment period over multiple trials in order to calibrate the upper and lower range of perceptual (and in the case of recognition, mnemonic) processing. As a result, the small number of trials present in our experiment might be insufficient for sequential dependencies to arise.

To address the issues outlined above, Experiment 3 used the recognition paradigm in an attempt to replicate and extend the work of Malmberg and Annis (2012; near-pairs condition) using three different categories of stimuli: photos of faces, photos of landscapes, and words. Accordingly, Experiment 3 had three main goals: (1) to extend previous research by testing for sequential dependencies on overall responding in face recognition, (2) if found, to determine if these sequential dependencies translate to predictable choosing behavior, and ( 3 ) to examine whether the strength of these effects varies across the testing phase.

We predicted sequential effects to arise across all three sets of stimuli. If sequential dependencies were observed for responses overall, we predicted that sequential effects would be stronger in the second half compared with the first half of testing blocks and thus also expected to be able to predict choosing behavior in late, but not early, test trials. Should sequential dependencies fail to appear for faces (vs. landscapes and words), it would not only undermine response-interference as a theory for the source of the sequential effects in recognition, but also suggest that sequential dependencies are unlikely to be problematic in the eyewitness context.

\section{EXPERIMENT 3}

\section{ETHICS STATEMENT}

This study was approved by the ethical review board of the Faculty of Psychology and Neuroscience of Maastricht University (ECP-160_03_01_2016). Participants in Experiment 3 provided consent by clicking the button to continue the experiment.

\section{PARTICIPANTS AND DESIGN}

One-hundred-fifty participants were recruited from online participation platforms. Five participants were excluded for the following reasons: failing two of the four control questions (1), failing to follow instructions (2), and taking a $20+$ min break in the middle of the first testing block (2). Participants with other anomalous data (e.g., low activity during the filler task) were flagged; When exploratory analyses to examine hit rates, false-alarm rates, accuracy, and choosing behavior did not reveal any of these flagged participants to 
be outliers, their data were retained for all further analyses. The average age of the remaining 145 participants was 22 years $(M=22.14, S D=6.49)$.

Participants were randomly assigned to one of three conditions to study paired stimuli of faces, landscapes, or words. For each study-test block, participants viewed 18 paired target stimuli during the learning phase and were tested on the 36 target stimuli and 36 fillers. Each participant took part in two study-test blocks, therefore each participant studied a total of 72 targets and was tested on a total of 144 stimuli (the 72 targets and 72 fillers). Participants were compensated with research participation credit if eligible, or otherwise not compensated.

METHOD

Materials. See Figure 3.1 for example stimuli.

Faces. Male and female faces with neutral expressions were selected from the Chicago Face Database (Ma, Correll, \& Wittenbrink, 2015). Faces that were particularly distinctive (i.e., shaved eyebrow, facial piercing, unique hair), were removed. Half of the target face stimuli were presented during the learning phase as same-gender pairs and half as opposite-gender pairs. (See Figure 3.1, Panel A)

Landscapes. Photographs of varied landscapes (e.g., sunsets, mountains, deserts, fields) were selected from the Places Scene Recognition Database (Zhou, Lapedriza, Xiao, Torralba, \& Oliva, 2014). Photos with particularly distinctive features (e.g., color filter) were not selected. (See Figure 3.1, Panel B)

Words. One thousand nouns were randomly chosen from the 5,000 most frequently used words according to the Corpus of Contemporary American English (Davies, 2008). Words were piloted for recognition by seven non-native English speakers whose nationalities are representative of the student population from which the sample is drawn (two Germans, two Belgians, and three Dutch). These non-native English speakers were asked to view the list of 890 nouns and remove those words that they did not recognize (i.e., would need to search for or translate). The stimulus pairs were randomly selected and paired from the remaining 813 nouns. (See Figure 3.1, Panel C)

Procedure. The procedure followed the procedure of Malmberg and Annis (2012; Experiment 1, near-pairs condition replications), with two exceptions. First, because the study was distributed online, a shape appeared at the end of each encoding block (block 1: blue star, block 2: black arrow), which was later used as a control question for attention. Second, due to availability of faces, participants studied only 36 total pairs (vs. 40 pairs in the original experiment) of the varying stimuli (faces, landscapes, or words). 


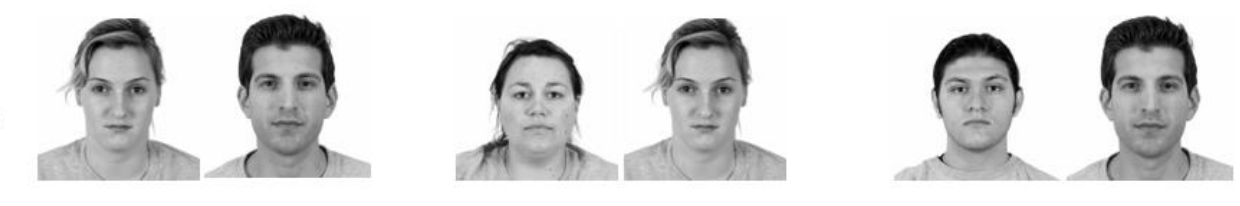

B
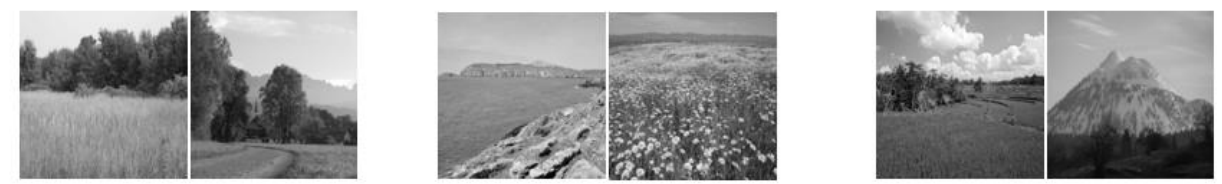

C

noon

bridge

officer

jazz

story

jacket

Figure 3.1 Experiment 3: Example stimuli pairs for faces (Panel A), landscapes (Panel B), and words (Panel C).

Participants were provided a link for the computer task. Participants in the face condition, for example, studied 18 pairs of faces (i.e., 36 faces total). Each pair was presented on screen for $2 \mathrm{~s}$ with a $0.1 \mathrm{~s}$ interstimulus interval. Following a $30 \mathrm{~s}$ distractor task (Pac-Man), participants were presented with two control questions asking them to indicate the form and color of the shape presented at the end of the encoding phase. Participants were then tested for their recognition of the previously-studied faces using the self-paced computer task. Participants in all conditions saw 36 target trials and 36 filler trials of never-before-seen stimuli presented at random, with the constraint that half of the pairs were tested consecutively and the other half were randomized into positions at least seven trials away from their corresponding target trials. Following another 1 min distractor task, this procedure was repeated for a second study-test block. At the end of the experiment, a final question prompted participants to describe the environment in which they completed the experiment (i.e., time of day, location, presence of others).

\section{RESULTS}

We focused on two types of analyses to address the three goals of the experiment. First, we conducted within-subjects tests on overall response patterns to replicate and extend those analyses conducted by Malmberg and Annis (2012). Accordingly, we conducted mixed Analyses of Variance (ANOVAs) on conditional hit rates, false-alarm rates, and choosing rates given previous responses and stimulus type (faces, landscapes, words). Because we were interested in how this effect might vary across the testing sessions, we conducted these same analyses on the conditional hit rates and false-alarm rates for the first half and second half of each of the two testing blocks. We refer to the first half of Block 
1 as Section 1, the second half as Section 2, and the first and second halves of Block 2 as Sections 3 and 4, respectively.

Second, we conducted between-subjects analyses in order to determine whether overall patterns would be reflected in individual choosing behavior. More specifically, we conducted logistic regressions analogous to those conducted in Experiments 1 and 2 to test whether we could predict choosing behavior on individual trials using target-presence and previous choosing as predictors. Although we present the results descriptively here, relevant statistics for within-subjects analyses can be found in Tables 3.3 and 3.4. Statistics for between-subjects analyses can be found in Table 3.5.

Table 3.3

Experiment 3: Results for ANOVAs and Follow-up Tests on Current Hit Rates, False-Alarm Rates, and Choosing Rates Given Previous Responses and Stimulus Type

\begin{tabular}{|c|c|c|c|c|c|c|}
\hline & $d f$ & $F$ & $\eta^{2}$ & $t$ & $d$ & $p$ \\
\hline \multicolumn{7}{|c|}{ ANOVAs: Faces, Landscapes, Words } \\
\hline \multicolumn{7}{|c|}{ Hit Rate: Hit, Miss, FA, CR } \\
\hline Previous Response & $2.25,321.58$ & 42.57 & .229 & & & $<.001$ \\
\hline Stimulus Type & 2,143 & 9.00 & .112 & & & $<.001$ \\
\hline Interaction & $4.50,321.58$ & 0.70 & .010 & & & 611 \\
\hline \multicolumn{7}{|c|}{ FA Rate: Hit, Miss, FA, CR } \\
\hline Previous Response & $2.47,355.40$ & 26.59 & .156 & & & $<.001$ \\
\hline Stimulus Type & 2,144 & 9.67 & .118 & & & $<.001$ \\
\hline Interaction & $4.94,355.40$ & 0.67 & .009 & & & .646 \\
\hline \multicolumn{7}{|c|}{ Choosing: Choose vs. Not } \\
\hline Previous Choose & 1 & 209.58 & .593 & & & $<.001$ \\
\hline Stimulus Type & 2 & 10.26 & .125 & & & $<.001$ \\
\hline Interaction & 2 & 7.42 & .093 & & & .001 \\
\hline Error (within Groups) & 144 & & & & & \\
\hline \multicolumn{7}{|c|}{ Follow-up t-tests: Choosing } \\
\hline Faces & 45 & & & 6.64 & 1.02 & $<.001$ \\
\hline Landscapes & 49 & & & 9.76 & 1.39 & $<.001$ \\
\hline Words & 50 & & & 9.13 & 1.30 & $<.001$ \\
\hline
\end{tabular}

Note. The top panel displays results for mixed ANOVAs on hit rates, false-alarm rates, and choosing rates with previous response as the within-subjects factor and stimulus type (faces, places, and words) as the betweensubjects condition. False alarm and correct-rejection are abbreviated here as FA and CR, respectively. The bottom panel examines the interaction between stimulus type and choosing rates using paired sample $t$-tests. Although sequential dependencies of choosing appeared in all three stimuli types, the effect was greatest for landscapes, followed by words, and then faces. 


\section{CHAPTER 3}

Table 3.4

Experiment 3: Results for ANOVAs on Current Hit Rates and False-Alarm Rates Given Previous Responses and Testing Section

\begin{tabular}{|c|c|c|c|c|}
\hline & $d f$ & $F$ & $\eta^{2}$ & $p$ \\
\hline \multicolumn{5}{|l|}{ Test sections $^{\mathrm{a}}: \mathbf{1}, \mathbf{2}, \mathbf{3}$, and 4} \\
\hline \multicolumn{5}{|l|}{ Hit Rate Contingencies } \\
\hline Previous Response & 3,318 & 41.22 & .280 & $<.001$ \\
\hline Test Section & $2.44,259.02$ & 8.63 & .075 & $<.001$ \\
\hline Interaction & $7.26,770.03$ & 0.47 & .004 & .860 \\
\hline \multicolumn{5}{|c|}{ False-Alarm Rate Contingencies } \\
\hline Previous Response & $2.73,305.63$ & 4.85 & .042 & .004 \\
\hline Test Section & $2.72,304.12$ & 9.06 & .075 & $<.001$ \\
\hline Interaction & $7.77,870$ & 0.98 & .009 & .447 \\
\hline
\end{tabular}

Note. The top panel displays results for repeated-measures ANOVAs on hit rates, false-alarm rates, and choosing rates with previous response (hit, miss, false alarm, correct rejection) and test section $(1,2,3,4)$ as the betweensubjects factors. aSections are broken down into: the first half of the first study-test block (Section 1 ), the second half of the first block (Section 2), and the first and second halves of the second block (Sections 3 and 4).

Table 3.5

Experiment 3: Results of Logistic Regression Predicting Choosing on Second and Third Recognition Test Trials Based on Previous Choosing and Target-Presence

\begin{tabular}{|c|c|c|c|c|c|c|c|}
\hline & \multirow[b]{2}{*}{$b$} & \multirow[b]{2}{*}{$S E$} & \multirow[b]{2}{*}{ Wald } & \multirow[b]{2}{*}{$p$} & \multicolumn{3}{|c|}{$95 \% \mathrm{Cl}$ for Odds Ratio } \\
\hline & & & & & Lower & Odds & Upper \\
\hline \multicolumn{8}{|l|}{ Section 1, trial 3} \\
\hline Choosing 1 & 0.57 & 0.37 & 2.37 & .124 & 0.86 & 1.76 & 3.62 \\
\hline Choosing 2 & 0.41 & 0.36 & 1.28 & .258 & 0.74 & 1.50 & 3.03 \\
\hline TP 3 & 1.62 & 0.38 & 18.13 & $<.001$ & 2.39 & 5.03 & 10.58 \\
\hline Constant & -0.97 & 0.36 & 7.41 & .006 & & 0.38 & \\
\hline \multicolumn{8}{|l|}{ Section 2 , trial 73} \\
\hline Choosing 71 & 0.18 & 0.38 & 0.22 & .640 & 0.57 & 1.20 & 2.52 \\
\hline Choosing 72 & 0.79 & 0.38 & 4.45 & .035 & 1.06 & 2.21 & 4.62 \\
\hline TP 73 & 0.98 & 0.38 & 6.68 & .010 & 1.27 & 2.67 & 5.61 \\
\hline Constant & -1.58 & 0.38 & 17.04 & $<.001$ & & 0.21 & \\
\hline \multicolumn{8}{|l|}{ Section 3 , trial 3} \\
\hline Choosing 1 & 0.42 & 0.38 & 1.22 & .269 & 0.72 & 1.52 & 3.20 \\
\hline Choosing 2 & -0.32 & 0.40 & 0.63 & .428 & 0.33 & 0.73 & 1.60 \\
\hline TP 3 & -0.70 & 0.36 & 3.69 & .055 & 0.24 & 0.50 & 1.01 \\
\hline Constant & 0.98 & 0.45 & 4.71 & .030 & & 2.67 & \\
\hline \multicolumn{8}{|l|}{ Section 4, trial 73} \\
\hline Choosing 71 & -0.55 & 0.38 & 2.12 & .145 & 0.28 & 0.58 & 1.21 \\
\hline Choosing 72 & 0.48 & 0.37 & 1.70 & .193 & 0.79 & 1.61 & 3.31 \\
\hline TP 73 & -0.66 & 0.36 & 3.43 & .064 & 0.26 & 0.52 & 1.04 \\
\hline Constant & 0.83 & 0.39 & 4.54 & .033 & & 2.30 & \\
\hline
\end{tabular}

Note: Variables were coded as follows. Choosing: non-choosing $=0$, choosing $=1$; target-presence: $\mathrm{TA}=0, \mathrm{TP}=1$. Section 1, Trial 3: $R^{2}=.14$ (Cox \& Snell) .19 (Nagelkerke). Model $\chi^{2}(3)=22.50, p<.001$; Section 2, Trial 73: $R^{2}=.07$ (Cox \& Snell) .10 (Nagelkerke). Model $\chi^{2}(3)=11.33, p=.010$. Section 3, Trial 3: $R^{2}=.03$ (Cox \& Snell) .05 (Nagelkerke). Model $\chi^{2}(3)=4.98, p=.173$; Section 4, Trial 73: $R^{2}=.05$ (Cox \& Snell) .07 (Nagelkerke). Model $\chi^{2}(3)$ $=11.33, p=.050 . \mathrm{Cl}=$ Confidence Interval. 
Preparation of data and calculation of contingency rates. Prior to calculating hit rates, false-alarm rates, and choosing rates, trials with response times faster than $200 \mathrm{~ms}$ were removed. This is because $\mathbf{2 0 0} \mathrm{ms}$ is the approximate threshold for recorded brain activity in response to human faces, as well as the earliest threshold for our ability to distinguish between familiar and unfamiliar faces (Bentin, Allison, Puce, Perez, \& McCarthy, 1996; Caharel, Ramon, \& Rossion, 2014). Hit rates were calculated as the proportion of correct answers on target-present trials and false-alarm rates were calculated as the proportion of incorrect answers on target-absent trials. Analyses used hit rates on the current trial (i) given that the previous trial ( $i-1)$ was a hit, miss, false alarm or correct rejection. Therefore, separate hit rate contingencies were computed for each participant for (a) hits that followed a hit, $H R_{h i t}=(H \mid i-1=h i t),(b)$ hits that followed a miss, $H R$ miss $=(H R$ | $i-1=$ miss), (c) hits that followed a false alarm and, (d) hits that followed a correct rejection. Analogous false-alarm rates for each participant were computed given that the previous response was a hit, miss, false alarm, or correct rejection (Malmberg \& Annis, 2012). Choosing rates were calculated as overall proportion of choosing (respond yes vs. no) on target-present and target-absent trials.

\section{Sequential effects as a function of stimulus type.}

Hit rate contingencies. We examined whether a hit on the current trial (i) was more or less likely given a hit, miss, false alarm, or correct rejection on the previous trial ( $i$ 1), and whether this relationship differed for our three types of stimuli: faces, landscapes, and words. Thus we conducted a mixed ANOVA with previous response being the withinsubjects factor, and type of stimulus being the between-subjects factor. There was a significant main effect of previous response. Planned contrasts indicated that a hit on the current trial was more likely if there was either a hit or false alarm compared with a correct rejection or miss on the previous trial. A hit on the current trial was also more likely if there was a correct rejection compared with a miss on the previous trial. The interaction of previous trial by type of stimulus was not significant. Thus, while we found sequential effects for hit rates, these effects did not differ significantly based on whether the stimuli were faces, landscapes, or words. See Figure 3.2 (Panel A).

False-alarm rate contingencies. We next examined whether a false alarm on the current trial (i) was more or less likely given a hit, miss, false alarm, or correct rejection on the previous trial $(i-1)$, and whether this relationship differed for our three sets of stimuli: faces, landscapes, and words. We conducted a mixed ANOVA with previous response being the within-subjects factor, and stimulus type being the between-subjects factor. There was a significant main effect of previous response, indicating that a false alarm on the current trial was more likely following a hit or false alarm (vs. miss or correct rejection) on the previous trial (see Figure 3.2, Panel B). There was no significant difference between hits vs. 
false alarms or misses vs. correct rejections. The non-significant interaction of previous trial with stimulus category provided no evidence that these effects differed significantly according to stimulus type.

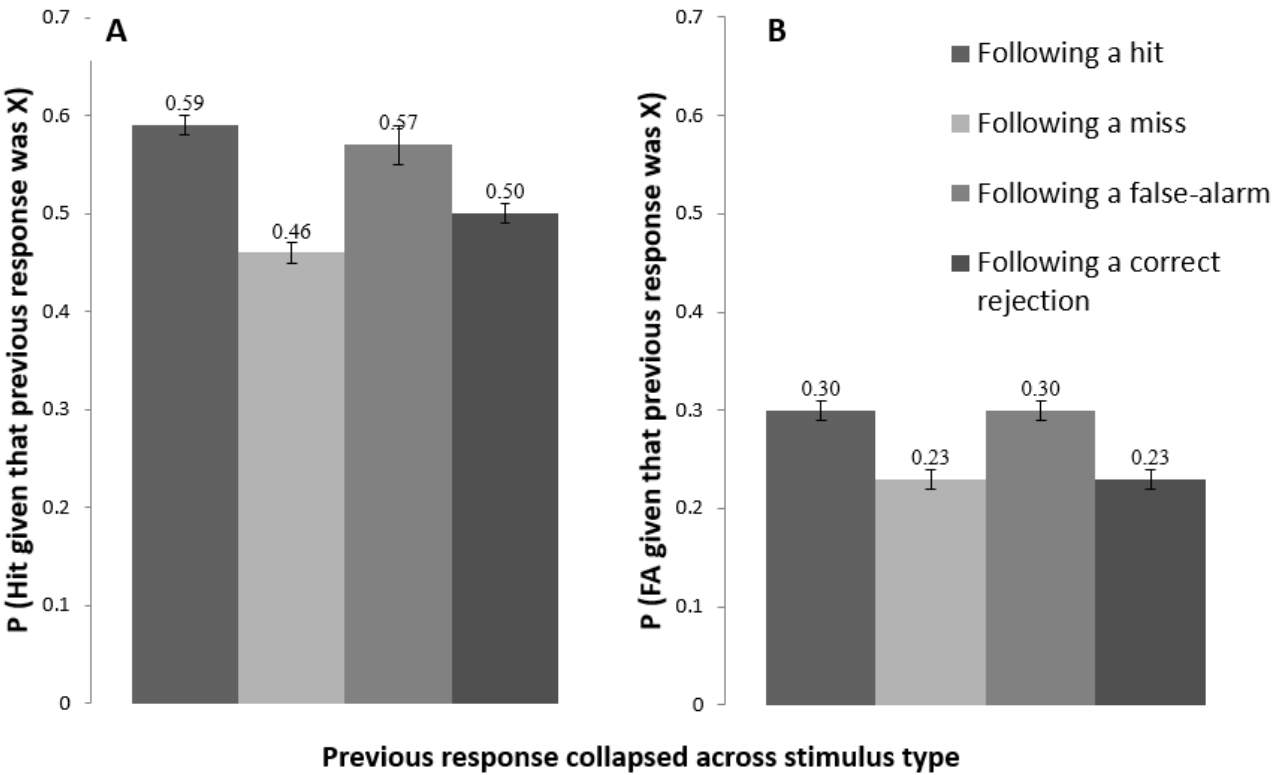

Figure 3.2 Experiment 3: Hit rate and false-alarm rate contingencies. Panel A displays the probability of a hit on the current trial given the previous response (hit, miss, false alarm, or correct rejection), collapsed across stimulus type (faces, landscapes, words). Previous responses of hit and false alarm do not significantly differ from each other, but all other comparisons are significant ( $p s<.001$ ). Panel B displays the probability of a false alarm on the current trial given the previous response, collapsed across stimulus type. A false alarm on the on the current trial is significantly more likely given a previous hit or false alarm when compared with a previous miss or correct rejections ( $p s<.001)$. Error bars reveal standard errors.

Sequential effects as a function of section. There were no significant interactions for test section (Sections 1, 2, 3, and 4) with previous response for hits, misses, false alarms, or correct rejections. There was a main effect of test section, such that Sections 1 and 3 displayed higher hit rates and lower false-alarm rates than Sections 2 and 4 . Section 1 also displayed higher false-alarm rates than Section 3. See supplementary materials for descriptive statistics (see appendix, Table A3.2).

Sequential effects in choosing. In these analyses, we ask a similar question in a different manner: overall, is choosing (saying yes) on the current trial, more or less likely if you chose or did not choose (said no) on the previous trial? We conducted mixed ANOVAs with previous choosing (choose vs. not choose) as the within-subjects factor and stimulus type as the between-subjects factor. There was a significant main effect of previous choosing, and a significant interaction between previous choosing and stimulus type. 
Although sequential dependencies arose within all stimulus categories, the effect was greatest for landscapes, followed by words, and then faces. Together, these results indicate that choosing on the current trial was more likely if the participant chose (vs. did not choose) on the previous trial and that this effect was weakest for face stimuli. See appendix for descriptive statistics (Table A3.3).

Predicting choosing on individual trials. Given that we successfully replicated analyses demonstrating sequential dependencies in overall recognition memory, including for faces, we subsequently tested whether those effects would translate to predictable behavior on individual trials over the course of the testing sessions. Therefore, we chose the first three trials of each testing block and the three middle trials of each block (Block 1 : trials 1-3 and 71-73; Block 2: trials 1-3 and 71-73). These analyses are of particular interest because they apply analyses from Experiments 1 and 2 to a dataset in which sequential dependencies have already been detected. We consider the first three trials of the first block a proxy for the three showup identification decisions in Experiments 1 and 2. We chose to test the middle trials rather than later trials in order to avoid isolating groups of responses likely to display fatigue effects. Analyses were analogous to Experiment 2 with one exception. Given that there was no current target-presence by previous choosing interaction in Experiment 2, this interaction was not included.

Choosing on the previous trial predicted choosing on the current trial for only one of the four analyses, and current trial target-presence predicted choosing in only trials of Block 1. Thus, despite finding that, in general, hits and false alarms were more common when participants chose on the previous trial, behavior on previous trials was not a useful predictor of choosing for these sets of individual trials.

\section{DISCUSSION}

Experiment 3 sought to replicate and extend previous work in sequential dependencies in recognition memory (Malmberg \& Annis, 2012). Except for online data collection and the inclusion of additional questions to control for attention, the procedure followed the near-pairs condition in Malmberg and Annis' Experiment 1. We expected that sequential dependencies would arise for recognition responses for all three types of stimuli, that these effects would be stronger in later portions of testing, and that this would be reflected in the capacity to predict current choosing from previous choosing in later, but not earlier, test trials.

As expected, the probability of a hit in the current trial (i) was higher if the previous response $(i-1)$ was also a hit compared with if the previous response was a miss. The probability of a false alarm on the current trial was increased if it was preceded by either a hit or a false alarm (compared with a miss or correct rejection). Noticeably, this pattern of 
results did not differ across category of stimuli, meaning we replicated Malmberg and Annis' results using pictures of landscapes and words, and extended those results to include face recognition. Taken together, these results demonstrate that the probability of saying yes on the current trial increases any time it is preceded by a yes on the previous trial, a conclusion reflected in the analyses conducted on choosing behavior.

We also conducted analyses to determine whether the relationship of previous response reported above changed over the course of the testing session. Contrary to predictions, the effect of previous response did not vary as a function of test section. Although accuracy displayed fatigue effects across the sections (higher accuracy in the first half of each testing block compared with the second half), the strength of sequential dependencies remained constant throughout. Essentially, sequential dependencies did not change across the length of testing.

Lastly, we tested whether these overall effects of sequential dependencies would translate to predictable behavioral outcomes on specific trials. We found little support for the idea that choosing on a previous trial predicted choosing on the current trial. Rather, while sequential dependencies did arise in overall choosing behavior across the total 288 trials, and even the 72 trials comprising each half of the testing blocks, these effects did not reliably arise as predictable choosing behavior on individual trials.

\section{GENERAL DISCUSSION}

This line of research aimed to answer this key question: in making a series of ostensibly independent showup identification decisions for different perpetrators, is the current decision of an eyewitness related to the previous one(s)? In Experiments 1 and 2, we addressed this question within the eyewitness identification paradigm. Participants watched a mock crime video with three perpetrators and were subsequently asked to make three showup identification decisions, one suspect for each of the perpetrators. Although we found some evidence for sequential dependencies in both experiments, the effect overall was not consistent. These unexpected results led us to question whether methodological differences between the recognition and eyewitness paradigm could explain the inconsistencies. In particular, we considered whether sequential dependencies would arise for recognition specifically for faces, and whether the number of trials tested influenced the ability to identify these dependencies. Thus, Experiment 3 replicated and extended Malmberg and Annis' (2012) research for sequential dependencies in recognition decisions to test whether (1) sequential dependencies would also arise for face recognition, (2) these effects could predict choosing behavior on individual trials, and (3) the strength of the above effects varied across the testing session. This approach allowed us to conduct both within-subjects testing to replicate previously reported effects in recognition memory, 
and the between-subjects modeling applied in Experiments 1 and 2. Experiment 3 showed that sequential dependencies do arise overall for face recognition decisions, that the strength of these effects remains consistent across the testing session, but that these effects do not reliably predict choosing behavior for individual trials. These results and their implications for theory and practice are discussed in turn.

\section{SEQUENTIAL DEPENDENCIES ARISE FOR FACE RECOGNITION DECISIONS}

In Experiment 3, we successfully replicated previous research, demonstrating that when participants make a series of yes/no recognition decisions, their responses are affected by the previous trial. A hit on the current trial was more likely when a hit or false alarm (vs. miss or correct rejection) on the previous trial occurred, and a false alarm on the current trial was more likely when either a hit or false alarm (vs. miss or correct rejection) occurred on the previous trial. To confirm this, analyses on choosing behavior established that choosing begets choosing: if participants said yes (vs. no) on the previous trial, the probability of saying yes on the current trial is increased. Notably, these effects were found for three types of stimuli, including images of faces. Indeed, in our analyses with hit rate and false-alarm rate contingencies, while the overall contingency rates varied depending upon the stimulus type, the relationship between previous and current response did not. Thus, this experiment adds to a growing list of decisions in which sequential dependencies arise, including detection of sound (Jones et al., 2006), ratings of sweetness in wine tastetests (Schifferstein \& Frijters, 1992), and judgements of frequency in landscape recognition (Annis \& Malmberg, 2013).

Experiment 3 suggests that we can rule out the possibility that the inconsistent effects within Experiment 1 and 2's eyewitness identification paradigm reflect the use of face stimuli rather than the words or landscapes used in previous research. By extension, this means that sequential dependencies could conceivably arise for someone making a series of yes/no decisions in person recognition settings, such as security personnel looking for banned football fans in a stadium or scanning the crowd for known-threats at political events. Indeed, this may be a useful setting in which to study sequential dependencies in applied recognition memory, and to consider in training security agents.

\section{EFFECTS ARE CONSISTENT ACROSS TESTING}

Next, we tested whether the strength of sequential dependencies varied across the length of the testing session. Accumulator models used to explain sequential dependencies predict shifts over time based on variation in criterion placement or accumulation starting points (e.g., SAMBA; Brown et al., 2008; Matthews \& Stewart, 2009). We hypothesized that effects would be stronger in the second half of each testing session compared with the first half of each session. Indeed, Schifferstein and Kuiper (1997) removed the first 20 "outlier" responses of their experiment tasting aqueous solutions 
because high response variability is greatest in these initial trials. Contrary to expectations, the strength of sequential dependencies remained constant across the length of the testing session.

In sum, our results established that sequential dependencies arise consistently within participants separately from individual differences in criteria. We could therefore be certain that our results replicated previous experiments on sequential dependencies as we transitioned to apply the regression models used in Experiments 1 and 2.

\section{SEQUENTIAL DEPENDENCIES ARE NOT REFLECTED AS PREDICTABLE CHOOSING BEHAVIOR}

We next tested whether these dependencies would also predict behavioral outcomes on the first three and middle three trials of each testing block. The first three trials of the first block are of greatest interest because they best represent the three showup identification trials in Experiments 1 and 2. Consistent with Experiments 1 and 2, and despite detecting sequential dependencies in overall data, we were not able to detect sequential dependencies in individual trials. Given that the strength of sequential dependencies detected by within-subjects analyses did not vary across the testing session, it was unsurprising to find that detecting sequential dependencies on individual trials did not differ. Critically, these results appear to be good news for the eyewitness context. We were originally concerned that multiple identification decisions may give rise to sequential dependencies, and thus affect the integrity of the identification decisions being made. However, this is not the case. If we cannot predict current recognition decisions from previous ones, then there is less reason to believe that dependencies are likely to be problematic for the multiple high-stakes recognition decisions in the eyewitness identification context.

These results are in line with a recent study that considers the effect of making multiple lineup decisions, although not within the theoretical framework of sequential dependencies (Mansour, Beaudry, \& Lindsay, 2017). In that study, participants watched 24 videos, each followed by lineup identification decisions on target-present and target-absent trials. The authors found that the number of trials had either no effect or a trivial effect on accuracy, choosing, or confidence. This is not to say that a series of identification decisions cannot possibly be related to each other. Indeed, sequential dependencies are only one way in which the assumption of independence may be violated between multiple decisions. Research on probability matching (Vulkan, 2000) demonstrates that people making a series of decisions use a response strategy that reflects their beliefs about base rates for the task. For example, students may avoid circling the option $A$ too many times on a multiple-choice test because they believe that correct answers are likely to be evenly distributed across the listed options. It is possible that eyewitnesses confronted with multiple lineups are influenced by these expectations of base rates (i.e., the police probably detained all three 
perpetrators vs. the experimenter would never show me all three perpetrators). In other words, though we have ruled out one possibility on the relationship between multiple identification decisions, there is more to be investigated.

\section{CONCLUSION}

Neither the use of faces nor differences in the number of trials could explain the contradictory results in Experiments 1 and 2 that we sought to resolve. However, the inability to use previous choosing behavior as a predictor for current choosing in Experiment 3, a data set that we know contains sequential dependencies, still serves to clarify our previous contradictions. We suspect that the discrepancy between detecting sequential dependencies in overall responses using within-subjects analyses and not on individual responses with regression models is an indication of weak effects. The within-subjects ANOVAs provide the statistical power to detect small effects, while the regression models do not. In this case, probabilities of choosing on current trials are heightened by previous choosing over hundreds of opportunities to choose or not to choose, but these effects do not translate to detectable behavioral outcomes of choosing on individual trials. In each of three experiments, it was sometimes possible to predict current choosing from previous choosing, but not reliably so, and often not in the expected direction.

In summary, sequential dependencies arise in face recognition, and though the accuracy across stimuli and sections of testing session may vary, the pattern of dependencies does not change. However, these effects did not translate to individual trials, and we therefore suggest that the integrity of identification and recognition decisions is not likely to be impacted by making the multiple decisions in a row. This is the first paper to systematically explore sequential dependencies in face recognition and particularly in eyewitness identification, contributing to the small, but vital, literature that aims to disentangle factors underlying the decreased performance in recognition for multiple faces. It thus contributes towards the eventual goal to offer procedural recommendations adapted to the difficulties present in the administration of identification procedures in the context of multiple perpetrator crimes. 


\section{APPENDIX: SUPPLEMENTARY MATERIAL}

Table A3.1

Pilot Study: Mean (Standard Deviation) Age, Distinctiveness, Memorability, Typicality and Similarity Values for Target Faces and Corresponding Innocent Suspect

\begin{tabular}{lcrrrrr}
\hline & Perpetrator 1 & Suspect 1 & Perpetrator 2 & Suspect 2 & Perpetrator 3 & Suspect 3 \\
& $M(S D)$ & \multicolumn{1}{c}{$M(S D)$} & \multicolumn{1}{c}{$M(S D)$} & \multicolumn{1}{c}{$M(S D)$} & \multicolumn{1}{c}{$M(S D)$} & \multicolumn{1}{c}{$M(S D)$} \\
\hline Age & $23.45(1.47)$ & $22.91(2.76)$ & $22.00(2.05)$ & $23.27(2.76)$ & $25.14(1.64)$ & $22.73(2.41)$ \\
Distinctiveness & $2.95(1.00)$ & $2.82(1.22)$ & $3.14(0.94)$ & $2.86(0.99)$ & $3.77(0.97)$ & $3.45(1.06)$ \\
Memorability & $3.00(1.07)$ & $2.59(1.14)$ & $2.00(1.16)$ & $2.73(0.83)$ & $3.50(1.01)$ & $3.27(0.99)$ \\
Typicality & $1.72(0.98)$ & $1.72(1.12)$ & $1.86(1.08)$ & $1.64(1.29)$ & $2.00(1.02)$ & $2.05(1.09)$ \\
Similarity & $2.81(0.80)$ & $2.45(0.96)$ & $2.45(0.91)$ \\
\hline
\end{tabular}

Note: Participants were shown each of the photographs (targets and replacements) individually and were asked to estimate age and to rate distinctiveness and memorability on a five-point scale from 1 (not at all distinctive/memorable) to 5 (extremely distinctive/memorable) and to rate deviation from typicality (How much would this face have to be modified to look completely typical/average?) on a scale from 0 (no modification) to 5. Participants indicated how similar they considered the two faces on a scale from 1 (not at all similar) to 5 (very similar). Innocent suspects were rated as statistically non-different to the perpetrator for the following three factors: memorability, distinctiveness, and deviation from typicality. Innocent suspects 2 and 3 significantly differed in age from their respective perpetrators: Suspect 2: $t(21)=2.73, p=.013$; Suspect $3: t(21)=6.41, p<$ .001. Perpetrators and their corresponding innocent suspects were also rated for similarity. These tests revealed no significant differences between pairs; $p s \geq .162$.

Table A3.2

Experiment 3: Hit Rates and False-Alarm Rates (Standard Error) Given Previous Response as a Function of Test Section

\begin{tabular}{|c|c|c|c|c|c|c|c|c|}
\hline & \multicolumn{4}{|c|}{ Hit Rate } & \multicolumn{4}{|c|}{ FA Rate } \\
\hline & Hit & Miss & FA & CR & Hit & Miss & FA & $\mathrm{CR}$ \\
\hline \multicolumn{9}{|l|}{ Section } \\
\hline & $.66(.02)$ & $.59(.02)$ & $.66(.02)$ & $.62(.02)$ & $.34(.02)$ & $.28(.02)$ & $.31(.02)$ & $.29(.01)$ \\
\hline & $.52(.02)$ & $.47(.02)$ & $.50(.03)$ & $.49(.02)$ & $.29(.02)$ & $.24(.02)$ & $.27(.02)$ & $.26(.02)$ \\
\hline & $.63(.02)$ & $.56(.03)$ & $.60(.03)$ & $.56(.02)$ & $.35(.02)$ & $.26(.02)$ & $.31(.02)$ & $.28(.02)$ \\
\hline & $.52(.02)$ & $.45(.02)$ & $.51(.03)$ & $.44(.02)$ & $.29(.02)$ & $.27(.02)$ & $.29(.03)$ & $.23(.02)$ \\
\hline
\end{tabular}

Note. Participants took part in two study-test blocks. Sections represent the first half of the first block (Section 1), the second half of the first block (Section 2), and the first and second halves of the second block (Sections 3 and 4). False alarms and correct rejections are denoted by FA and CR, respectively.

Table A3.3

Experiment 3: Choosing Rates (Standard Error) Given Previous Choosing as a Function of Stimulus Type

\begin{tabular}{lll} 
& & \multicolumn{2}{c}{ Choosing Rates } \\
\cline { 2 - 3 } Section & Choose & Not Choose \\
\cline { 2 - 3 } Faces & & $.51(.01)$ \\
Landscapes & $.58(.01)$ & $.47(.01)$ \\
Words & $.57(.01)$ & $.50(.01)$ \\
\hline
\end{tabular}


SHOWUP IDENTIFICATIONS FOR MULTIPLE PERPETRATORS 
CHAPTER 4 
CHAPTER 4

\section{Face value: Testing the utility of contextual face cues for face recognition}

Tupper, N., Sauer, J., Sauerland, M., Fu, I., \& Hope, L. (2018). Face value: Testing the utility of contextual face cues for face recognition. Manuscript accepted pending minor revisions. 


\section{ABSTRACT}

The presence of multiple faces during a crime may provide a naturally-occurring contextual cue to support eyewitness recognition for those faces later. Across two experiments (Experiments 4 and 5 of this thesis), we sought to investigate mechanisms underlying previously-reported cued recognition effects, and to determine whether such effects extended to encoding conditions involving more than two faces. Participants studied sets of individual faces, pairs of faces, or groups of four faces. At test, participants in the singleface condition were tested only on those individual faces without cues. Participants in the two- and four-face conditions were tested using no cues, correct cues (a face previously studied with the target test face), or incorrect cues (a never-before-seen face). In Experiment 5, associative encoding was promoted by a rating task. Neither hit rates nor false-alarm rates were significantly affected by cue type or face encoding condition in Experiment 4, but cuing of any kind (correct or incorrect) in Experiment 5 appeared to provide a protective buffer to reduce false-alarm rates through a less liberal response bias. Results provide some evidence that cued recognition techniques could be useful to reduce false recognition, but only when associative encoding is strong. 
The recognition and identification of an unfamiliar face is a difficult task, and becomes even more challenging as the number of unfamiliar faces to be remembered increases. Indeed, accuracy rates for face recognition and identification decrease when there are multiple faces to study (Clifford \& Hollin, 1981). Although divided attention at encoding may play a role in this effect (e.g., Bindemann, Burton, \& Jenkins, 2005; Bindemann, Jenkins, \& Burton, 2007), this does not entirely account for the multiple-face recognition disadvantage. Accuracy decreases even when participants have unlimited time to encode the presented faces and when divided attention is controlled for (Bindemann, Sanford, Gillatt, Avetisyan, \& Megreya, 2012). The persistence of the multiple-face disadvantage suggests that the drop in accuracy is caused, at least in part, by an increase in memorial demand when attempting to hold two faces in memory for any period of time (Bindemann et al., 2012).

Interestingly, the presence of multiple faces not only increases memorial demand, but also provides a naturally-occurring contextual cue that may promote recognition. Early experiments demonstrated increased recognition rates for a previously-studied face when that face, having been paired with another face at study, was presented with its corresponding pair at test (rather than when presented alone or with a different face; e.g., Watkins, Ho, \& Tulving, 1976; Winograd \& Rivers-Bulkeley, 1977, see below). It is possible that the benefits of cued recognition could be used to support eyewitness memory in the context of multiple perpetrator crimes, whereby the face of the first perpetrator might enhance the eyewitness' ability to accurately identify the second perpetrator in a lineup. However, experiments attempting to apply cuing in the context of eyewitness identification for multiple perpetrator crimes do not align with these theoretical predictions. In one experiment, for example, eyewitnesses saw a two-perpetrator crime and subsequently viewed two sequential lineups presented side-by-side: Eyewitnesses viewed one picture from the lineup of the perpetrator next to one picture from the lineup of the accomplice, and this was repeated until all of the photos of each lineup had been shown. Compared with the traditional sequential and simultaneous lineups, this adapted cuing lineup improved accuracy in rejecting innocent suspects from a perpetrator-absent lineup (i.e., decreased false alarms), but not in identifying actual perpetrators (i.e., increasing hits; Wells \& Pozzulo, 2006). In another experiment, eyewitnesses of a two-perpetrator crime viewed a simultaneous lineup of the perpetrator with a single photo of the accomplice presented next to it as a cue, or vise-versa (Dempsey, 2012). Compared with when no cue was presented, this technique improved the correct rejection of innocent suspects for the perpetrator, but not the correct rejection of innocent suspects for the accomplice.

It is possible that results of these adapted lineups did not demonstrate the benefits of cued face recognition because of a mismatch in converting this theoretically informed 
technique to practice. These results may also be due to the original experiments not having been designed with an eyewitness context in mind, therefore failing to control for potentially confounding variables that exist in such a context. To address these limitations (discussed in detail below), it would be useful to re-examine what we understand about cued face recognition with an eye towards its eventual application in the context of eyewitness identification for multiple perpetrator crimes. Therefore, we sought to replicate previously-reported enhancing effects in cued face recognition, to determine whether such effects could extend to include more than two faces (given that many crimes do involve more than two perpetrators; Hobson, Wilcock, \&Valentine, 2012; Horvath \& Kelly, 2009; Sandholtz, Langton, \& Planty, 2013), and to investigate the mechanisms underlying those effects. We compared the traditional cued encoding condition in which participants study paired faces, with two additional encoding conditions: a control condition where participants encoded single faces and another experimental condition where participants encoded groups of four faces.

\section{CUED RECOGNITION AND FACES}

Memory researchers have long known that context matters for retrieval. Contextual cues are often implemented to help individuals recall seemingly-forgotten details in episodic memory (encoding specificity principle; Thomson \& Tulving, 1970), including to facilitate eyewitness recall during investigative interviews (context reinstatement; Geiselman, Fisher, MacKinnon, \& Holland, 1986). It has been hypothesized that these cues work because the retrieval of a memory is dependent upon the way it was stored, and an item in episodic memory is, by nature, nested within our experience of the relevant event (Polyn, Norman, \& Kahana, 2009; Tulving \& Thomson, 1973). Thus, episodic memory is not only tied to temporal markers (when an event occurred), but can also be integrated with memory traces for the context of the event. For example, we often recall items in semantic clusters (i.e., related words like book, hardcover, paperback, bestseller) as a result of our long-standing associations between items in our experience, but also in source clusters as a result of the associations we form during study phase between items (i.e., information from a friend vs. the internet; Polyn et al., 2009). A contextual cue at retrieval takes advantage of the associative nature of memory whereby, for example, peripheral details of the environment can cue additional pathways for the retrieval of critical details.

Contextual cues also benefit recognition memory, including recognition for faces (e.g., Watkins et al., 1976). A variety of external contexts have been shown to enhance recognition for target faces, including backgrounds on which the faces were studied, eyewitness descriptions of targets, clothing worn by targets, and other faces presented with the target faces (see Davies, 1988 for a review). For example, Winograd and Rivers-Bulkeley 
(1977) asked participants to memorize pairs of faces during the study phase-one of which served as the target face while the other served as the cue. During the test phase, participants were presented with a target face alongside either a face they had previously studied (correct cue), a face that had not been previously studied (incorrect cue), or no cue at all. In this forced-choice paradigm, recognition performance for target faces was enhanced by the presentation of correct cue faces and impaired by incorrect cue faces, while performance with no cues fell in-between. Similarly, Watkins and colleagues (1976) demonstrated reduced hit rates for face recognition when they implemented substituted cues: previously-studied faces that had been paired with another face during study. In other words, simply swapping context, as opposed to introducing new context, also affected face recognition performance.

Although the above experiments found an enhancing effect of using correct cues for face recognition, other research has found no such effect (e.g., Bower \& Karlin, 1974) or found that hit rates were undermined by incorrect cues, but not enhanced by correct cues (Kan, Giovanello, Schnyer, Makris, \& Verfaellie, 2007). A number of issues relating to encoding and recognition may underpin these discrepant results. First, the context must be strongly encoded in association with the target for recognition (Peris, 1985; as cited in Davies, 1988). If the participant did not pay attention to the contextual information, or did not link it with the target information, then presenting the cue at test will not improve recognition. Second, it appears that context is useful as a recognition memory cue only when other, stronger cues are lacking (Smith \& Vela, 2001). Some theories of recognition hold that recognition is comprised of two mechanisms (e.g., Mandler, 1980). The first is the perceptual system that is automatically activated and produces fast answers that hinge on the feeling of familiarity. The second is the cognitive system, which is activated when the first does not immediately provide an answer. The second system is slower and searches for external information, like context, to aid the response. This process is also captured in the rationale of the outshining hypothesis, which contends that we use the most relevant cues available to recognize faces. When our memory trace is strong, that memory outshines the utility of environmental context. However, for weak memory traces, such as when there was suboptimal encoding or longer retention intervals, context may support memory to improve performance (Smith \& Vela, 2001).

\section{THE CURRENT RESEARCH}

Cued recognition presents an interesting means of enhancing face recognition, a concept that may prove useful to the applied fields of eyewitness identification or wantedpersons recognition. The second face in a two-perpetrator crime provides a naturallyoccurring context for the eyewitness, and one that is particularly relevant for humans. Given 
our natural tendency to orient attention towards other human faces, a second face may increase the chance of incidental associative encoding (Di Giorgio, Turati, Altoè, \& Simion, 2012). Thus, face cues may support eyewitnesses of a multiple perpetrator crime while viewing a suspect lineup. This is not an entirely novel idea; at least two published experiments (Hobson \& Wilcock, 2011; Wells \& Pozzulo, 2006) and one unpublished dissertation (Dempsey, 2012) have attempted to apply face cuing to support eyewitness memory and adapt identification procedures in the context of multiple-perpetrator crimes. However, none of these attempts provided convincing evidence that this method of cuing memory could aid lineup identification decisions, and it is of interest to understand why this might be. The current research aims to understand the disconnect between a theoretically intriguing mnemonic device and the poorly-understood difficulties in applying such a device; particularly by extending it to explore boundary conditions and orienting the effects. These goals are explored further below.

To begin, previous experiments showing inconsistent effects of face cues expose concerns for the replicability of a cued face recognition effect. Relevant experiments were mostly conducted in the 1970s, and it is only decades later that we are now interested in using this research for applied contexts (i.e., eyewitness identification). However, original studies made decisions that are incongruent with contemporary methodological standards in psychology. For one, the relevant research reports methodologies with confounding variables. For example, Winograd and Rivers-Bulkeley (1977) specifically paired malefemale pairs according to overt compatibility, with particular care to maintain similar ages between them. Further, participants rated perceived compatibility of the couples, further enhancing the romantic link between the pairs. It is possible that this likeness between the pairings provided contextual information for correct and incorrect cuing. A participant, for example, might correctly recognize a target face of a confident-looking 20-year-old white male paired with a confident-looking 20-year-old white female because the pairing enhances recognition. It might also be because the nature of the pairing gives the participant a hint as to the correct answer given prior knowledge that the two faces presented are an intuitively compatible couple. By extension, a participant might be worse at recognizing that same male when paired with an older, shy-looking female because the participant intuits they are not a compatible couple. Likewise, previous experiments using face pairs did not randomize the left-right orientation of those pairs such that if the target face was on the right side of the screen during the encoding phase, it was also presented on the right side during test (Watkins et al., 1976; Winograd \& Rivers-Bulkeley, 1977). It may be that the boost in hit rates is sensitive to this spatial context as well as contextual cuing. The recent movement in psychological science to replicate previous effects stems in part from a realization that the field now has updated knowledge on methodological issues like 
sample sizes, randomization, and experimenter influence that have changed the way we conduct experiments (i.e., Simmons, Nelson, \& Simonsohn, 2011). Given the intriguing theoretical rationale for the effects, but inconsistent results obtained, we aimed to replicate the cued recognition effect isolated from other influences such as the context effects of placement, or intuitive responses based on overt compatibility of couples. Therefore, we randomized left-right location of target faces during encoding and randomly paired or grouped photographs of male faces selected from a large database.

Furthermore, we sought to place the cued face recognition effects in context by comparing them with traditional recognition memory, whereby single faces are encoded and single faces are tested; for simplicity, we refer to this as orienting the effects of cuing because we aim to understand how memory tested in a cued recognition paradigm compares to memory tested in a traditional, non-cued recognition paradigm. In the cued face recognition paradigm, researchers use correct cues to reconstruct encoding context, incorrect cues to represent a change in context, and no cues as control trials. However, not presenting a cue at test is also a change of context because the absence of context is itself a deviation from the original context. It is therefore unclear whether previously-reported effects reflect a benefit of correct cuing, a detriment of incorrect cuing, or both. To accommodate this, we included a new control condition in which participants encoded two or four faces in the encoding phase, but were not presented with cues during test phase. Figure 4.1 shows a graphic representation of encoding and test conditions. This control condition (Figure 4.1, panel A) allowed us to examine the impact of number of faces at encoding (Figure 4.1, panel B, C). It may be that recognition rates without cues are equivalent across single-face and multiple-face encoding conditions. Thus, any benefit of correct cuing, for example, would be an enhancement over general face recognition. By contrast, if the no-cue condition varies between the single-face and multiple-face conditions, then any benefit of correct cuing would be compensation for increased difficulties.

Existing research also exposes questions regarding the potential boundary conditions for such an effect. For example, do beneficial context effects vary according to the number of additional faces to be encoded? Research on cued face recognition to date has held encoding conditions constant while manipulating the conditions at retrieval (e.g., Watkins et al., 1976; Winograd \& Rivers-Bulkeley, 1977). However, pairing two faces at encoding represents the minimum number of faces individuals might encode when attempting to implement cued face recognition. Limiting our consideration to pairs of faces fails to reflect the variability in group sizes that individuals encounter every day, and it is unclear if the benefits identified in previous work extend to conditions in which participants 
need to encode more stimuli. In other words, if four faces are studied together, can one of those faces effectively serve as a cue for another?

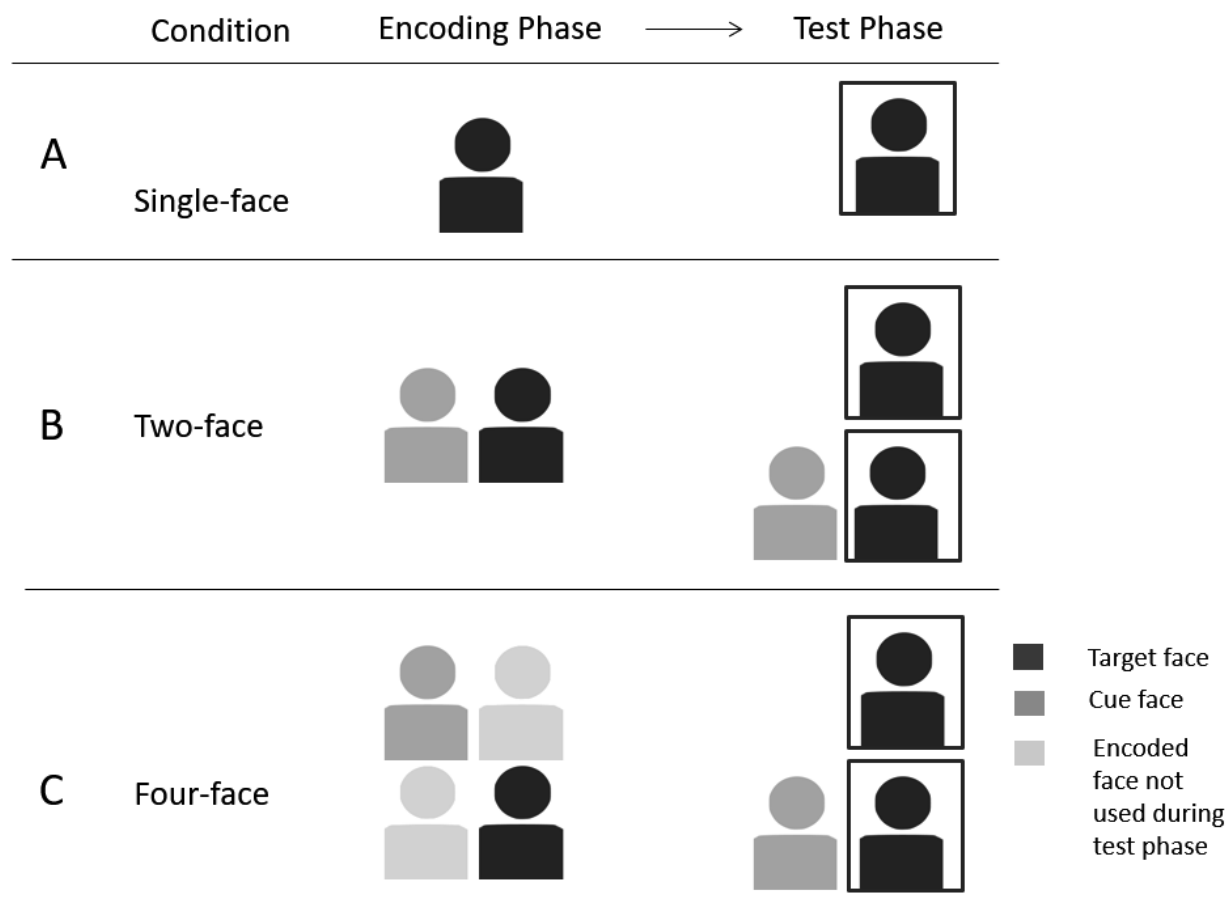

Figure 4.1. Experiments 4 and 5: Procedure for encoding and testing. Participants studied single faces, pairs of faces, or groups of four faces during the encoding phase. Participants in the single-face condition (Panel $A$ ) were only tested on individual faces (i.e., no-cue trials). Participants in the two-face (Panel B) and four-face condition (Panel C) were tested on trials with no cue and trials with either a correct cue or incorrect cue. Here, we demonstrate in conditions when, during the test phase, both the target face is present and the cue face is correct. However, participants were also tested on target faces that were absent and with cues that were incorrect. Color coding is used to demonstrate how cues and targets were chosen, but pariticpants did not know which faces during the encoding phase would be used as targets or cues. During the test phase, a box indicated the target face participants should report whether or not they recognized. Targets and cues were chosen at random from the left or right position of the encoding phase, but were always presented on the right and left, respectively, during the test phase.

There are several reasons why cuing with four-face stimuli may not be as effective as with two-face stimuli. First, there is the potential for generalized deficits in memory with the increased cognitive load associated with having more faces (e.g., four vs. two) to encode. Cuing in this case may be helpful, but with a general trend for the four-face condition to have decreased accuracy. Or cuing could be less effective because there is too diminished of a memory to support. Second, when faces are always presented at study as pairs, a correct cue also represents a complete cue. However, if four faces are presented 
during encoding and only one face is used to cue the recognition of the target face, this may represent a partial cue. Therefore, providing only one of the set of the associated cues at test may show a weaker effect. Third, there is the potential that associations between multiple (i.e., more than two) faces may not be encoded equally, and therefore may not be equally effective at retrieval. While two faces viewed side-by-side provide only one association that can be formed at encoding, four faces provide multiple associations that can be formed with varying strengths. Thus, the face chosen by experimenters to be a contextual cue may not be the one that was most strongly associated with the target at encoding. By contrast, it may be that each face is one of several possible cues, meaning that any of the faces would be sufficient to enhance recognition.

\section{EXPERIMENTS 4 AND 5}

These applied and theoretical considerations converge into three questions for the current research: (1) do previously-reported effects of contextual cuing replicate with contemporary methodology? (2) how do cuing effects vary as a function of the number of cues to be encoded? and ( 3 ) do these effects reflect a benefit of correct cuing, a detriment of incorrect cuing, or both?

Experiment 4 was a first attempt to establish to what extent correct cuing is beneficial or compensating for increased cognitive demand, and also whether this effect endures when cognitive load is increased by viewing multiple (i.e., more than two) faces at once. However, because results yielded minimal effects of either face encoding condition or cue type, we added a manipulation to more strongly encourage associative encoding between faces in Experiment 5. Thus, Experiment 5 was a direct replication of Experiment 4 , with the addition of the manipulation during the encoding phase (see procedure for further details). In both Experiments 4 and 5, we expected to replicate previous effects of cued face recognition in the two-face condition, and that those effects would extend to the four-face condition, though weaker.

\section{METHOD}

\section{PARTICIPANTS}

A total of 159 participants were recruited from Maastricht University. Three failed to follow instructions and were excluded from data analysis. The remaining 156 participants (121 women) in Experiments $4(n=78)$ and $5(n=78)$ were between the ages of 18 and 43 $(M=22.14, S D=3.09)$. Participants were compensated with either participation credit or a $5 €$ (Experiment 4 ) or $7.50 €$ (Experiment 5) voucher. These studies were approved by the standing ethical committee of the Faculty of Psychology and Neuroscience (ECP160_03_01_2016). 


\section{DESIGN}

We used a 3 (encoding group size: single face, two faces, four faces) x 3 (cue type: no cue, correct cue, incorrect cue) x 2 (target face presence: old, new) mixed design, with encoding group size as the between-subjects factor. Participants were randomly assigned to one of the three between-subjects conditions. Cue type was randomly ordered and target faces were determined at random. Two versions of each experiment were created in which the order of trials for all conditions was varied, and in the two- and four-face conditions, different target faces and cue faces were selected.

MATERIALS

Face stimuli. For each experiment, 204 photographs of male faces were used from a database of faces at Flinders University (Adelaide, Australia), the Psychological Image Collection at Stirling (pics.stir.ac.uk), and the AR Face Database (Martinez \& Benavente, 1998). Of those, 36 faces were used as target faces and the remaining 168 faces were used as correct cue faces at test, incorrect cue faces, or filler faces during encoding. Faces with features that were highly distinctive (e.g., piercings, unique haircuts, facial hair) were replaced. All photographs were in full color on a computer screen using Microsoft PowerPoint 2010. Each face image was 3.13 × 3.13 in. In the single-face condition, faces were placed in the center of the screen. In the two-face condition, two faces were placed side by side. In the four-face condition, the faces were presented in a $2 \times 2$ matrix. To create the multiple faces conditions, the faces were randomly grouped into pairs (two-face condition) or groups of four faces (four-face condition). Images of faces were not repeated between encoding blocks. Target faces were the same photographs at both encoding and test. See Figure 4.2 for example stimuli for each of the three encoding conditions.

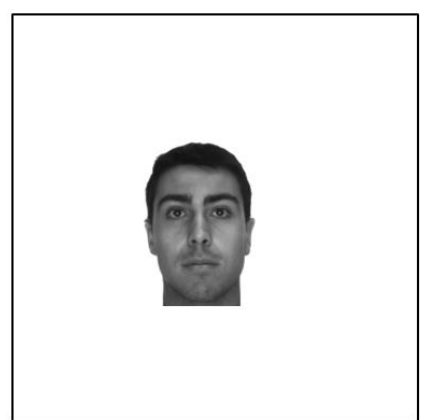

Single-face condition

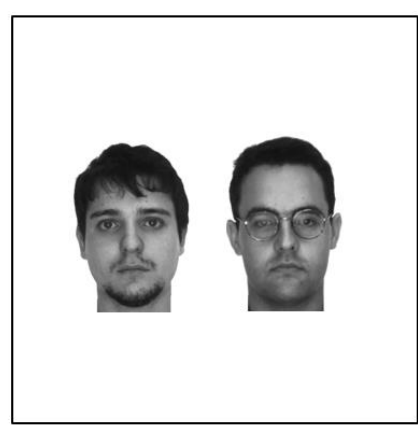

Two-face condition

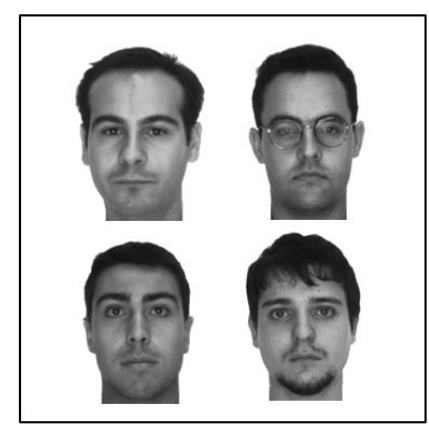

Four-face condition

Figure 4.2. Example encoding stimuli using images from AR Face database. 


\section{METHODOLOGICAL CONSIDERATIONS}

In comparing effects across conditions with only single faces and groups of four faces, there were two major methodological concerns: participant allocation of attention during encoding, and the number of total trials encoded. Each of these are addressed below.

Allocation of attention. We considered how increasing the number of faces on a given trial would impact the allocation of attention at encoding. Bindemann and colleagues (2012) demonstrated that given two stimuli, participants generally allocate their attention evenly between the stimuli. Unsurprisingly, identification performance was positively correlated with the amount of time spent studying the appropriate target face. Thus, the amount of attention devoted to any individual stimulus would be reduced if encoding duration remained constant, but the number of to-be-encoded stimuli would increase. We attempted to attenuate this concern by providing comparative encoding durations per face, rather than per trial. Participants in all conditions were allotted $2 \mathrm{~s}$ per face, even if those faces are presented in pairs or as a group. Thus, the exposure duration for single-face, twoface and four-face trials were 2,4 , and $8 \mathrm{~s}$, respectively. Participants were instructed to focus on all faces, but whether participants actually allocated attention equally across stimuli was beyond our control.

Encoding load. In order to compare the single-, two- and four-face conditions, we needed participants to complete the same number of test trials. However, this presents a problem at encoding. A participant who studied 36 trials of individual faces in the encoding phase will be tested on 72 trials of individual faces (half old, half new faces) in the test phase. Participants in the two-face and four-face conditions should therefore also confront 72 test trials with old and new faces. However, to test for cued face recognition, the 72 target faces at test require extra faces at encoding to be used as cues. The natural solution to this is to hold constant the number of encoding trials so that all conditions study 36 trials at encoding. Yet 36 trials in a two-face condition and a four-face condition consequently mean that a participant studies 72 faces and 144 faces at encoding, presenting extreme differences in memory load. In short, if we hold constant the number of test trials, we cannot also control for both the number of encoding trials and the total number of faces studied.

Our solution was to create testing blocks so that participants only ever studied 36 faces in each block: 36 individual faces, 18 paired faces, and 9 groups of four faces. They were then tested on 72,36 , and 18 old and new faces in the different conditions. The twoand four-face conditions repeated this study-test cycle until they had also completed 72 test trials, meaning the two-face condition encountered two study-test blocks and the four-face condition encountered four study-test blocks. Breaks in between the blocks were included in order to compensate for the memory load induced by viewing more faces. 


\section{PROCEDURE}

Experiment 4. Participants arrived in the lab to take part in individual testing sessions. Before the experiment, participants took part in a tutorial with three encoding trials and three test trials. Faces presented during the tutorial were not used during the actual experiment.

At encoding, participants were asked to memorize a number of faces. They were instructed to pay attention to the faces and that each face would be displayed for $2 \mathrm{~s}$ (if in the one-face condition), or that each set of faces would be displayed for $4 \mathrm{~s}$ (if in the twoface condition), or $8 \mathrm{~s}$ (if in the four-face condition). Encoding trials advanced automatically after the given interval and a fixation cross appearing for $500 \mathrm{~ms}$ between trials. Between the encoding and testing phases, participants performed a short arithmetic task for $30 \mathrm{~s}$. At test, each trial was presented for $5 \mathrm{~s}$, after which time the program automatically advanced to display a fixation cross. Participants were instructed to judge a test face, as either being old (previously-studied) or new (never-before-seen). If the participant indicated an 'old' response, they were also asked to indicate their confidence on a scale from 1 (not at all confident) to 7 (very confident) ${ }^{5}$. Participant responses were self-paced while the fixation cross was displayed, so that participants pressed the spacebar on the keyboard to advance to the next trial. Response latencies were not recorded.

In each condition, only one of the faces presented during the encoding phase was used as a target face at test. Participants in the single-face condition completed one studytest block, consisting of 36 single face stimuli during the encoding phase, and 72 single face trials at test (including the 36 study faces as targets and 36 previously unseen faces; these are referred to as old and new faces, respectively).

Single-face condition. During the encoding phase, participants viewed 36 individual photos of faces. Each face (all target faces) was shown for a duration of $2 \mathrm{~s}$. As for all conditions, each trial was followed by an interstimulus interval (ISI) of $500 \mathrm{~ms}$ before the next trial appeared. During the testing phase, participants completed 72 single face trials, half of which were old faces and half of which were new.

Two-face condition. During the encoding phase, participants viewed 18 pairs of faces. Each pair (one target face and one cue face) was shown for $4 \mathrm{~s}$ (allowing $2 \mathrm{~s}$ per face). No information was provided to participants on which of the two faces they would be tested, but participants were told to focus on all faces. During the testing phase, participants completed 36 trials: 12 were presented with no cue, 12 with a correct cue (a previouslystudied face), and 12 with an incorrect cue (a never-before-seen face). Presence of the target face was manipulated so that half of the trials were old, and half were new. The target

\footnotetext{
${ }^{5}$ Data available upon request from first author. Note that due to error in data collection, confidence data were only recorded for choosers.
} 
face was randomly selected from the pairs of faces for each of the two versions of the testphase. At test, the target face was always shown on the right side of the screen and always designated by a green square. This process was repeated with 18 new pairs of faces during encoding. Thus, there were two study-test blocks.

Four-face condition. This condition involved four blocks of trials. During the encoding phase, the participants viewed nine groups of four faces. Each stimulus group (one target face and three potential cue faces) was shown for $8 \mathrm{~s}$ ( $2 \mathrm{~s}$ per face). Participants were instructed to focus on all faces during encoding. During the testing phase, participants completed 18 trials: six trials were presented with no cue, six trials with a correct cue, and six trials with an incorrect cue. Presence of the target face was manipulated so that half of the trials were old, and half were new. The target face was randomly selected from the groups of faces and was always presented on the right side of the screen during test and designated by a green square. This process was completed three more times with nine new groups of four faces for each encoding phase.

Experiment 5. Experiment 5 was a direct replication for Experiment 4 except for the addition of a manipulation during the encoding phase to strengthen associative encoding. Participants were told that the experiment was to test the supposed relationship between initial impressions of how criminal the defendant looks, the memorability of their faces, and the number of years a single convicted defendant or a group of convicted defendants were sentenced to prison. After each stimulus was presented during the encoding stage, participants were asked to rate on a Likert scale how many years (4-10) the person(s) presented had been sentenced to prison by the judge. For example, in the fourface condition, participants were instructed, "All members of the pair were sentenced to the same number of years in prison. If you mark the number ' 4 ', it means that the person on the top left was sentenced to 4 years in prison, and the person on the top right, the bottom left, and the bottom right were each sentenced to 4 years in prison." This manipulation led to a second difference between Experiments 4 and 5. In Experiment 5, the encoding trials ISIs automatically advanced throughout the encoding phase. In Experiment 5, although the encoding trials automatically advanced after $2 \mathrm{~s}$ per face, the ISI was self-paced so that participants pressed the spacebar on the keyboard to advance to the next stimulus.

\section{DATA ANALYSIS}

Hit rates were defined as the probability of a correct response given that the stimulus $(S)$ is present (i.e., the trial shows an old face) and were calculated by dividing the number hits on target present trials by the total number of target present trials. The falsealarm rate was the probability of an incorrect response given that the stimulus is absent (i.e., the trial shows a new face) and were calculated by dividing the number of false alarms on target absent trials by the total number of absent trials. 
We computed signal detection statistics to test for effects on sensitivity and response bias ( $d^{\prime}$ and $c$, respectively). In the current research, sensitivity is the capacity to discriminate between old and new faces, which can range from 0 to 4.65 . A score of 0 indicates participants cannot discriminate at all, while 4.65 is considered an effective ceiling for discrimination (Macmillan \& Creelman, 2005). Response bias is the tendency to respond old or new regardless of whether the face is actually old or new. Scores can range from 2.33 to +2.33 . In our experiments, negative scores reflect the tendency to say "old" which is considered liberal response bias, and positive scores reflect the tendency to say "new", which is considered a conservative response bias (Macmillan \& Creelman, 2005).

In order to examine recognition performance in the no-cue condition, we tested hit rates and false-alarm rates across all face conditions (in single-, two-, four-face). Given that the single-face condition does not have correct- and incorrect-cues, only the two- and four-face groups were considered when comparing these cue-types.

\section{RESULTS}

\section{PREPARATION OF DATA}

Data were screened for outliers and normality prior to analysis. Shapiro-Wilk tests were used to assess normality for each of the conditions. In Experiment 5, one outlier was removed from the four-face condition because of an abnormally high false-alarm rate. When normality was violated, both nonparametric (Kruskal Wallis $H$ and Mann-Whitney $U$ ) and standard inferential tests (one-way Analyses of Variance [ANOVAs] and $t$-test) were conducted on hit rates and false-alarm rates. Results did not differ as a result of test, therefore ANOVAs and $t$-tests are reported throughout. Where relevant, we conducted Bayesian analyses - run in JASP (2017) software - to determine whether the data provided evidence of equivalence. We use Jarosz and Wiley's (2014) interpretations of Bayes Factors as evidence for the alternative hypothesis, in which they report descriptive thresholds provided by Jeffreys (1961) and Raftery (1995). Approximate cut-offs are as follows: 1-3 constitutes weak evidence for the null or alternative hypothesis, 3-10 is positive/substantial evidence; $10-20$ is strong/very strong evidence; $20+$ is very strong/decisive ${ }^{6}$. Descriptive statistics of hit and false-alarm rates as a function of cue type and face group are reported in Table 4.1. Sensitivity and response bias rates as a function of cue type and face group are reported in Table 4.2. Inferential statistics are not reported in text, but are available in Tables 4.3-4.5.

\footnotetext{
${ }^{6}$ For ease of interpretation, $B F_{10}$ is used to designate the Bayes factor as evidence in favor of the alternative hypothesis and $B F_{01}$ is used to designate the Bayes factor as evidence in favor of the null hypothesis.
} 


\section{REPLICATING AND EXTENDING EFFECTS OF CUED FACE RECOGNITION}

First, we tested for the replication of previous effects of cued face recognition, and whether these effects vary as a function of the number of faces to be encoded. Therefore, we conducted 2 (encoding condition: two-face, four-face) x 3 (cue type: no, correct, incorrect) mixed ANOVAs on hit rates and false-alarm rates with cue type as the withinsubjects variable (see Table 4.3 for inferential statistics). We expected that correct cues would enhance hit rates compared with no cues and incorrect cues. Although we also expected to see false-alarm rates rise with correct cues (vs. no cues or incorrect cues), we did not expect these to eliminate the enhancing effects of correct cuing for recognition.

Hit rates. In both Experiments 4 and 5, there were no significant main effects of face encoding condition or cue type on hit rates; the interaction was also non-significant. Thus, we found no evidence that either the number of faces at encoding or the type of cue presented at test affected participants' hit rates. Because we wanted to test evidence in favor of the null hypothesis, we conducted Bayes analyses using JASP (JASP Team, 2017). In these analyses, models are presented for (i) each main effect, (ii) the combined main effects, and (iii) the combined main effects with the interaction of the main effects. The strength of evidence for each model is then compared against the strength of evidence for the null hypothesis. Bayes analyses provided strong support for the null hypothesis in both experiments, with the model including both main effects and the interaction resulting in a Bayes factor $\left(B F_{01}\right)$ of 233.55 or higher when compared with the evidence in favor of the null. In other words, none of the models (including individual main effects, combined main effects, or combined main effects and the interaction) fit the data better than the null hypothesis. 


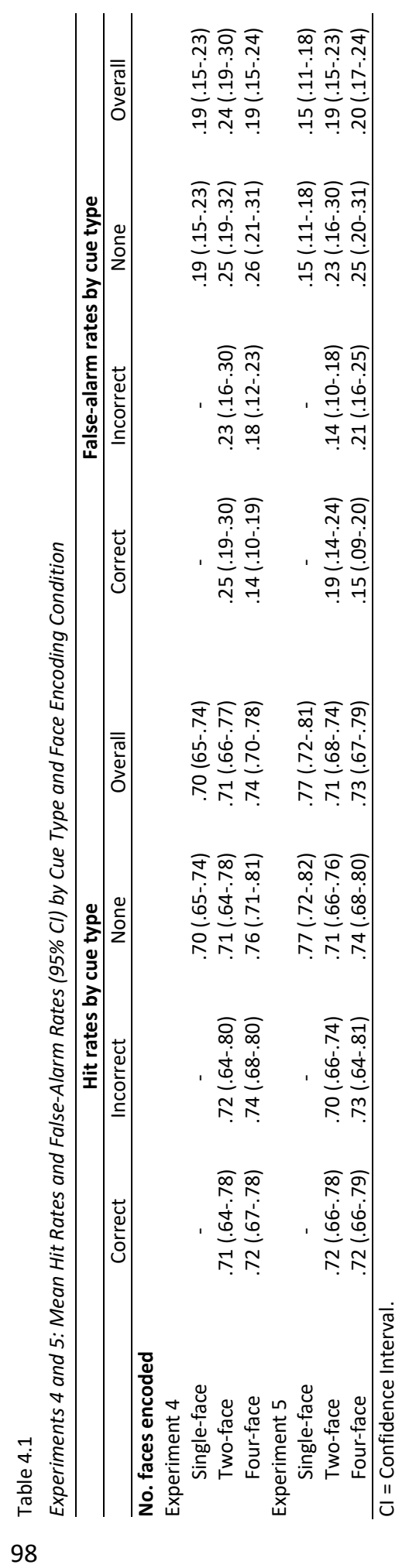




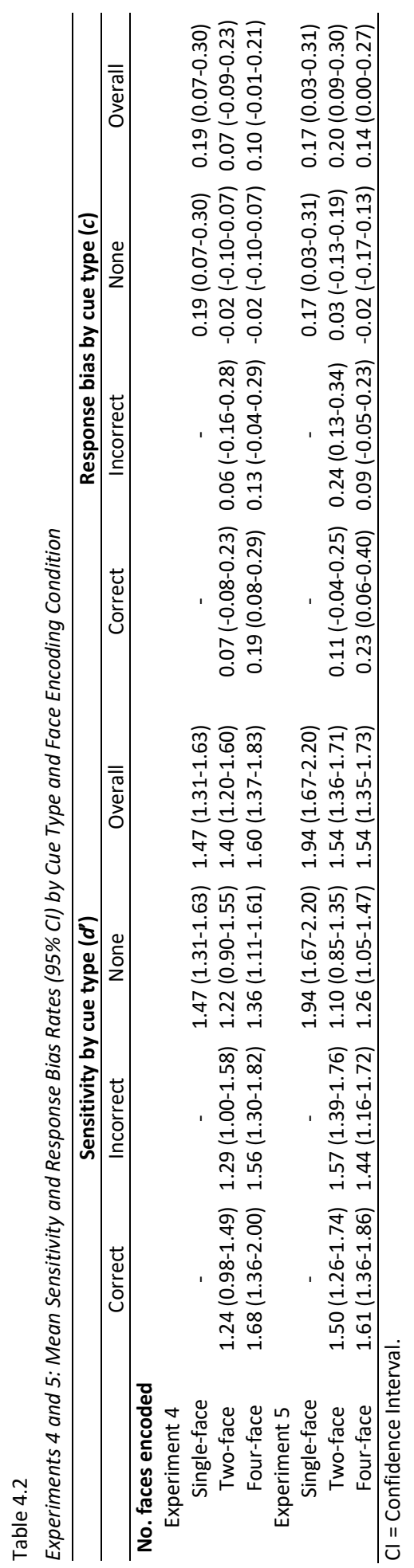




\section{CHAPTER 4}

Table 4.3

Experiments 4 and 5: Results for ANOVAs and t-tests Comparing Hit Rates and False-Alarm Rates Across Cue Types and Face Encoding Condition

\begin{tabular}{|c|c|c|c|c|c|c|}
\hline & $d f$ & $F$ & $\eta^{2}$ & $t$ & $d$ & $p$ \\
\hline \multicolumn{7}{|l|}{ Experiment 4} \\
\hline \multicolumn{7}{|l|}{ Orient the effect of cuing } \\
\hline \multicolumn{7}{|c|}{ One-way ANOVAs (No. faces; $1,2,4$ ) } \\
\hline $\mathrm{HR}$ & 2,75 & 1.69 & .043 & & & .191 \\
\hline FAR & 2,75 & 1.81 & .046 & & & .171 \\
\hline \multicolumn{7}{|c|}{ Replicate and extend cuing effects } \\
\hline \multicolumn{7}{|c|}{ Mixed ANOVAs 2 (No. faces: $2 v 4$ ) $\times 3$ (cue type) } \\
\hline \multicolumn{7}{|c|}{$\mathrm{HR}$} \\
\hline Cue & 2,100 & 0.27 & .005 & & & .762 \\
\hline No. faces (2v4) & 1,50 & 0.61 & .012 & & & .439 \\
\hline Interaction & 2,100 & 0.41 & .008 & & & .666 \\
\hline \multicolumn{7}{|l|}{ FAR } \\
\hline Cue & 2,100 & 4.74 & .087 & & & .011 \\
\hline No. faces (2v4) & 1,50 & 2.26 & .043 & & & .139 \\
\hline Interaction & 2,100 & 3.23 & .061 & & & .044 \\
\hline \multicolumn{7}{|c|}{ Follow-up simple main effects } \\
\hline \multicolumn{7}{|c|}{ Paired sample $t$-tests (No. faces: $2 \mathrm{v} 4$ ) } \\
\hline No cue & 50 & & & 0.15 & .04 & .881 \\
\hline Correct & 50 & & & 2.72 & .78 & .007 \\
\hline Incorrect & 50 & & & 1.21 & .33 & .234 \\
\hline \multicolumn{7}{|l|}{ Experiment 5} \\
\hline \multicolumn{7}{|c|}{ Orient the effect of cuing } \\
\hline \multicolumn{7}{|c|}{ One-way ANOVAs (No. faces; $1,2,4$ ) } \\
\hline $\mathrm{HR}$ & 2,74 & 1.10 & .029 & & & .337 \\
\hline $\begin{array}{c}\text { FAR } \\
\end{array}$ & 2,74 & 3.95 & .096 & & & .023 \\
\hline \multicolumn{7}{|c|}{ Replicate and extend cuing effects } \\
\hline \multicolumn{7}{|c|}{ Mixed ANOVAs 2 (No. faces: $2 v 4$ ) $\times 3$ (cue type) } \\
\hline \multicolumn{7}{|c|}{$\mathrm{HR}$} \\
\hline Cue & 2,98 & 0.10 & .002 & & & .904 \\
\hline No. faces (2v4) & 1,49 & 0.35 & .007 & & & .556 \\
\hline Interaction & 2,98 & 0.18 & .004 & & & .833 \\
\hline \multicolumn{7}{|l|}{ FAR } \\
\hline Cue & $\begin{array}{r}1.78 \\
87.12\end{array}$ & 5.89 & .107 & & & .005 \\
\hline No. faces (2v4) & 1,49 & .316 & .006 & & & .577 \\
\hline Interaction & $\begin{array}{r}1.78 \\
87.12\end{array}$ & 2.96 & .057 & & & .063 \\
\hline
\end{tabular}

Note. $t$-tests are two-tailed. HR and FAR represent hit rates and false-alarm rates, respectively. 
Table 4.4

Experiments 4 and 5: Results for Bayesian ANOVAs Comparing Hit Rates and False-Alarm Rates Across Cue Types and Face Encoding Condition

\begin{tabular}{|c|c|c|c|c|c|}
\hline Models & $\mathrm{P}(\mathrm{M})$ & $\mathrm{P}(\mathrm{M} \mid$ data $)$ & BF $\mathrm{m}$ & $\mathrm{BF}_{10}\left(\mathrm{BF}_{01}\right)$ & $\%$ error \\
\hline \multicolumn{6}{|l|}{ Experiment 4} \\
\hline \multicolumn{6}{|l|}{ Orient the effects of cuing } \\
\hline \multicolumn{6}{|c|}{ One-way ANOVAs (No. faces; 1, 2, 4) } \\
\hline \multicolumn{6}{|l|}{$\mathrm{HR}$} \\
\hline Null model & 0.50 & 0.71 & 2.45 & 1.000 & \\
\hline No. faces & 0.50 & 0.29 & 0.41 & $0.41(2.45)$ & 0.01 \\
\hline \multicolumn{6}{|l|}{ FAR } \\
\hline Null model & 0.50 & 0.69 & 2.24 & 1.000 & \\
\hline No. faces $(1,2,4)$ & 0.50 & 0.31 & 0.45 & $0.45(2.24)$ & 0.01 \\
\hline \multicolumn{6}{|c|}{ Replicate and extend effects of cuing } \\
\hline \multicolumn{6}{|c|}{ Mixed ANOVAs 2 (No. faces: $2 \mathrm{v} 4)$ x 3 (cue type) } \\
\hline \multicolumn{6}{|c|}{$\mathrm{HR}$} \\
\hline Null model & 0.20 & 0.68 & 8.51 & 1.00 & \\
\hline Cue & 0.20 & 0.06 & 0.23 & $0.08(12.36)$ & 0.80 \\
\hline No. faces (2v4) & 0.20 & 0.24 & 1.27 & $0.35(2.82)$ & 1.27 \\
\hline Cue $+2 v 4$ & 0.20 & 0.02 & 0.08 & 0.03 (32.99) & 2.91 \\
\hline Cue + 2v4 + Cue $* 2 v 4$ & 0.20 & $<0.01$ & 0.01 & $.004(233.55)$ & 1.72 \\
\hline \multicolumn{6}{|l|}{ FAR } \\
\hline Null model & 0.20 & 0.11 & 0.48 & 1.00 & \\
\hline Cue & 0.20 & 0.30 & 1.72 & $2.83(0.35)$ & 0.80 \\
\hline No. faces $(2 v 4)$ & 0.20 & 0.08 & 0.32 & $0.70(1.42)$ & 0.80 \\
\hline Cue + 2v4 & 0.20 & 0.22 & 1.13 & $2.07(0.48)$ & 2.35 \\
\hline Cue $+2 v 4+$ Cue $* 2 v 4$ & 0.20 & 0.30 & 1.68 & $2.81(0.36)$ & 2.12 \\
\hline
\end{tabular}

Experiment 5

Orient the effects of cuing

One-way ANOVAs (No. faces; 1, 2, 4)

HR

Null model

0.50

$0.79 \quad 3.80$

1.00

No. faces $(1,2,4)$

0.50

$0.21 \quad 0.26$

$0.26(3.80)$

0.03

FAR

Null model

$\begin{array}{lll}0.50 & 0.31 & 0.44\end{array}$

1.00

No. faces $(1,2,4)$

0.50

$0.70 \quad 2.28$

$2.28(0.44)$

0.01

Replicate and extend effects of cuing

Mixed ANOVAs 2 (No. faces: $2 \mathrm{v} 4$ ) $\times 3$ (cue type)

HR

$\begin{array}{lrrrrr}\text { Null model } & 0.20 & 0.71 & 9.90 & 1.000 & \\ \text { Cue } & 0.20 & 0.05 & 0.21 & 0.07(14.23) & 0.83 \\ \text { No. faces (2v4) } & 0.20 & 0.22 & 1.12 & 0.31(3.25) & 1.02 \\ \text { Cue + 2v4 } & 0.20 & 0.02 & 0.07 & 0.02(43.48) & 5.84 \\ \text { Cue + 2v4 + Cue*2v4 } & 0.20 & <0.01 & <0.01 & <0.01(353.33) & 2.62 \\ \text { AR } & & & & & \\ \text { Null model } & 0.20 & 0.07 & 0.30 & & \\ \text { Cue } & 0.20 & 0.58 & 5.50 & 8.21(0.12) & 0.96 \\ \text { No. faces (2v4) } & 0.20 & 0.02 & 0.08 & 0.27(3.75) & 1.14\end{array}$




\begin{tabular}{|c|c|c|c|c|c|}
\hline Models & $\mathrm{P}(\mathrm{M})$ & $\mathrm{P}(\mathrm{M} \mid$ data $)$ & BF $\mathrm{m}$ & $\mathrm{BF}_{10}\left(\mathrm{BF}_{01}\right)$ & $\%$ error \\
\hline Cue $+2 v 4$ & 0.20 & 0.15 & 0.72 & $2.16(0.46)$ & 1.00 \\
\hline Cue $+2 v 4+$ Cue $* 2 v 4$ & 0.20 & 0.18 & 0.87 & $2.54(0.39)$ & 1.24 \\
\hline
\end{tabular}

Note. HR and FAR represent hit rates and false-alarm rates, respectively. $\mathrm{BF}_{10}$ refers to evidence in favor of the alternative hypothesis. In parentheses, $B F_{01}$ refers to evidence in favor of the null hypothesis. $\left(B F_{01}=1 / B F_{10}\right)$. $B F_{M}$ refers to the change between prior (P[M] and posterior $P[M \mid$ data] model odds (see Wagenmakers et al., 2017 for in-depth explanation of Bayesian hypothesis testing).

Table 4.5

Experiments 4 and 5: Results for t-tests and ANOVAs Comparing Sensitivity and Response Bias as a Factor of Face Encoding Condition or Cue Type

\begin{tabular}{|c|c|c|c|c|c|c|c|}
\hline & $d f$ & $F$ & $\eta^{2}$ & $t$ & $d$ & $p$ & $\mathrm{BF}_{10}\left(\mathrm{BF}_{01}\right)$ \\
\hline \multicolumn{8}{|c|}{ Experiment 4} \\
\hline \multicolumn{8}{|c|}{ Two vs. four-face conditions } \\
\hline \multirow[t]{2}{*}{ Sensitivity (d') } & 49 & & & - & -0.36 & .207 & $0.55(1.83)$ \\
\hline & & & & 1.28 & & & \\
\hline \multirow[t]{2}{*}{ Response bias (c) } & 43.97 & & & - & -0.10 & .728 & $0.30(3.39)$ \\
\hline & & & & 0.35 & & & \\
\hline \multicolumn{8}{|c|}{ Cue type (no, correct, incorrect cue) } \\
\hline$d^{\prime}$ & 2,60 & 0.74 & .024 & & & .482 & $0.18(5.71)$ \\
\hline$c$ & $1.60,47.98$ & 2.17 & .067 & & & .135 & $0.53(1.87)$ \\
\hline \multicolumn{8}{|c|}{ Experiment 5} \\
\hline \multicolumn{8}{|c|}{ Two vs. four-face conditions } \\
\hline \multirow[t]{2}{*}{ Sensitivity (d') } & 48 & & & - & -0.02 & .949 & $0.28(3.53)$ \\
\hline & & & & 0.06 & & & \\
\hline Response bias (c) & 48 & & & 0.71 & 0.20 & .488 & $0.35(2.88)$ \\
\hline \multicolumn{8}{|c|}{ Cue type (no, correct, incorrect cue) } \\
\hline$d^{\prime}$ & 2,54 & 3.23 & .107 & & & .047 & $1.43(0.70)$ \\
\hline$c$ & 2,54 & 4.71 & .149 & & & .013 & $4.26(0.24)$ \\
\hline
\end{tabular}

Note. We computed signal detection statistics to test for effects on sensitivity and response bias ( $d^{\prime}$ and $c$, respectively). We used $t$-tests to compare across the two-face and four-face conditions. We used repeated measures ANOVAs across the no, correct, and incorrect cue conditions. The single-face condition was excluded due to the difference in cue types. $\mathrm{BF}_{10}$ refers to evidence in favor of the alternative hypothesis. In parentheses, $\mathrm{BF}_{01}$ refers to evidence in favor of the null hypothesis.

False-alarm rates. In Experiment 4, there was a significant main effect of cue that was modified by a significant interaction between face encoding condition and cue. Inspection of simple main effects showed that on correct cue trials, participants in the twoface condition had significantly more false alarms relative to the four-face condition. However, these effects should be interpreted cautiously. While we report the results of the interaction because of the significant $p$-value, Bayesian analyses provided weak support for the interaction $\left(B F_{10}=2.81\right)$. All other comparisons were non-significant.

In Experiment 5, the main effect of cue type was significant and Bayes analyses provided substantial evidence in support of a meaningful effect $\left(B F_{10}=8.21\right)$. Collapsing across encoding face condition, participants produced more false alarms on no-cue trials compared with correct- or incorrect-cue trials, though there was no significant difference between the incorrect-cue trials and the correct-cue trials. The main effect of face encoding 
condition was not significant and Bayes analyses provided evidence in favor of the null hypothesis $\left(B F_{01}=3.75\right)$.

Sensitivity and response bias. We used $t$-tests to compare sensitivity and response bias across the two- and four-face conditions (see Table 4.5 for inferential statistics). In Experiments 4 and 5, there were no significant effects. In Experiment 4, Bayesian analyses only provided sufficient evidence to support the null hypothesis for response bias $\left(B F_{01}=\right.$ 3.39), but not sensitivity $\left(B F_{01}=1.83\right)$. In Experiment 5 , Bayesian analyses only provided sufficient evidence to support the null hypothesis for sensitivity $\left(B F_{01}=3.53\right.$ ), but not response bias $\left(B F_{01}=2.88\right)$.

We used repeated measures ANOVAs to compare sensitivity and response bias across the no-, correct-, and incorrect-cue trials. In Experiment 4, there were no significant differences between groups for either sensitivity or response bias, and there was sufficient evidence to support the null hypothesis for sensitivity $\left(B F_{01}=5.71\right)$. In Experiment 5 , however, response bias did change as a result of cue type $\left(B F_{10}=4.26\right)$. Participants had a negative response bias on no-cue trials (i.e., were more likely to be biased to respond old) compared with trials with correct and incorrect cues. In Experiment 5, sensitivity also changed as a result of cue type such that participants had lower sensitivity (i.e., were less able to discriminate old from new faces) on no-cue trials compared with correct or incorrect cue trials. However, Bayesian analysis provided only weak evidence for this effect $\left(B F_{10}=\right.$ 1.43).

\section{ORIENTING THE EFFECT OF CUING}

Next, we tested whether previously-reported effects reflect a benefit of correct cuing, a detriment of incorrect cuing, or both. To address this, we tested the effect of group size on the no-cue trials on hit rates and false-alarm rates, using one-way ANOVAs with three levels (face encoding condition: one, two, or four faces). This allows us to understand the initial differences in how number of faces at encoding impacts recognition performance.

Hit rates. In both Experiments 4 and 5, there were no significant differences in hit rates between face encoding conditions. This means that there was no evidence of any difference between the single-, two-, or four-face encoding conditions when no cue was presented during test. Bayesian analyses showed weak support for the null hypothesis in Experiment $4\left(B F_{01}=2.45\right)$, but moderate support for the null hypothesis in Experiment 5 $\left(B F_{01}=3.80\right)$.

False-alarm rates. For Experiment 4, there were again no differences in false-alarm rates between groups. Bayesian analyses did not provide compelling evidence to support the null hypothesis $\left(B F_{01}=2.24\right)$. For Experiment 5 , however, there was a significant difference between face encoding conditions, though Bayesian analyses provided only weak support for this $\left(B F_{10}=2.28\right)$. The single-face condition had significantly fewer false alarms 
compared with the four-face condition. The false-alarm rate for the two-face condition fell between, and was neither significantly greater than the single-face nor significantly smaller than the four-face condition.

\section{SUMMARY OF KEY FINDINGS}

In Experiment 4, neither hit rates nor false-alarm rates were impacted by the type of cue provided at test (correct, incorrect, no cue). On no-cue trials, face encoding condition had a statistically significant but negligible effect on false-alarm rates, such that the fourface condition produced a higher false-alarm rate than the single-face condition. In Experiment 5, which included an additional manipulation designed to promote associative encoding, cuing again had no impact on hit rates, but reduced false alarms. This effect was present regardless of the face encoding condition.

\section{DISCUSSION}

Previous research has shown that when faces are studied in pairs, one of those faces can be used at test to cue recognition of the other. Across two experiments, we sought to answer the following three questions in this cued face recognition domain: (1) do previously reported effects replicate with updated methodology? (2) how do cuing effects vary as a function of the number of cues to be encoded? and (3) do these effects reflect a benefit of correct cuing, a detriment of incorrect cuing, or both? While relevant research has only used pairs of faces at encoding, we aimed to extend previous investigations of this effect by adding two conditions (single-face control, four-face condition) to the encoding phase. Specifically, the single-face condition was added to establish baseline differences in studying one vs. multiple faces, thus orienting the effects of cuing. The four-face condition was added to determine whether the effect would also arise in situations in which people encode more than two faces at a time. At test, participants in the single-face condition were shown individual faces that had either been previously studied (old) or had never been studied before (new). Participants in the two-face or four-face encoding conditions were shown either a target face alone (no cue), or a target face presented alongside a correct or incorrect cue face. Experiment 5 was a replication of Experiment 4, but with the addition of a rating task to strengthen the associative encoding of the multiple faces.

Experiment 4 provided little evidence with which to answer our research questions, and it is only in Experiment 5, when we added a manipulation to enhance associative encoding, that cued recognition effects arose. This alone suggests that cuing effects are likely to require more than the arbitrary pairing of stimuli at encoding. Accordingly, we consider Experiment 4 as a demonstration of the inconsistency of cued recognition effects, the likely need for a meaningful link to encourage associative encoding between stimuli, and as a caution while interpreting the findings of Experiment 5 . However, 
we focus primarily on the results of Experiment 5 in order to discuss the results in light of our three research aims. First, our results did not replicate previously-reported cued face recognition effects. Contrary to expectations, in both experiments, cuing did not influence hit rates. However, in Experiment 5, cuing with either correct or incorrect cues (vs. no cues) did reduce false-alarm rates. Therefore, while cuing enhanced overall accuracy, this occurred through reducing the likelihood of falsely recognizing a new face. Second, these effects did extend to scenarios with more faces, such that cuing reduced false alarms in conditions in which participants studied pairs of faces and groups of four faces. Third, our results provide some evidence that cuing helps to reduce the disadvantage of studying multiple faces (vs. individual faces) by reducing response bias. These results are discussed in turn.

\section{REPLICATION OF CUED FACE RECOGNITION EFFECTS}

First, we consider whether we could replicate previous effects in cued face recognition. The two-face condition mimicked the original research (Watkins, Ho, \& Tulving, 1976; Winograd \& Rivers-Bulkeley, 1977) in cued face recognition, but our methodology differed in two important ways. First, we randomized the left-right placement of the target faces between study and test, such that placement effects could not exert an influence on participant responses. Second, we randomized pairing of faces so that likeness between the faces (i.e., age, impression of personality, etc.) could not provide clues to correct answers. Following cued recognition and the encoding specificity principles (Thomson \& Tulving, 1970), we expected in both experiments to see hit rates increase when a correct cue was presented compared with either an incorrect cue or no cue at all. However, this was not the case. Hit rates were not affected by cuing, but false-alarm rates decreased in response to both correct and incorrect cuing. In line with these results, we also found response bias decreased when any context was shown, whether it was correct or incorrect context. This means that participants became more conservative in their responses, and were thus less likely to respond "old" when cues were presented. It may be that cuing, regardless of the veracity of the cue, signaled participants to remember to reject in light of uncertainty, or that cuing increased the strength of evidence (i.e., the sense of familiarity or availability of memory) participants require for a positive response. However, these explanations would need to be explored in future research.

It is interesting to note that the failure to increase hit rates arose in an experiment when face context was isolated from the possibility of placement context and intuitive impressions of couples belonging together. The encoding specificity principle asserts that contextual information is more important than semantic information in cuing (Thomson \& Tulving, 1970); If contextual cuing is useful for face recognition, randomized groupings of faces should not theoretically reduce the effect of correct cuing as long as the faces are 
encoded as context. Therefore, we would expect to still see increased hit rates as a result of correct cuing, and reduced hit rates as a result of incorrect cuing. By contrast, Bayesian analyses for both experiments provided strong evidence that hit rates did not differ as a result of cuing.

Instead, the observed cuing-related enhancement in accuracy was a result of reduced false-alarm rates. Thus, cuing did not enhance the true recognition of old faces, but did enhance the ability to reject a new face that was not previously studied. Cued recognition studies typically find that correct cues increase false alarms, but not enough to outweigh the benefits of cuing context (see Davies, 1988). However, our results diverge from previous patterns of contextual cuing, such that false-alarm rates actually decreased as a result of cuing. We should note that it is not unusual for a manipulation in memory research to affect hits and false alarms to different degrees. For example, context reinstatement in eyewitness identification research often increases the false-alarm rate rather than the hit rate (Shapiro \& Penrod, 1986). However, the current research is closely aligned to the previous experiments in cued face recognition. This raises the question why, within the same field of cued recognition research, cues of faces would not present previously-reported risks of increasing false alarms and why it would conversely reduce the false recognition of new faces. Because both correct and incorrect context reduced the false-alarm rate, and because sensitivity was minimally affected by cue type, it would be difficult to argue that memory was enhanced as a result of cuing. However, response bias did become more conservative any time context was present, meaning that participants needed more evidence to report the face as old, which was reflected in the reduced falsealarm rate.

\section{EXTENDING CUING EFFECTS TO MORE THAN TWO FACES}

Next, we consider whether such cuing effects extend to contexts in which more than two faces are encoded at the same time. This finding was straightforward. While the four-face condition appeared to be at a slight disadvantage compared with the two-face group when no cue was presented, contextual cuing was equally useful in both groups to reduce the false-alarm rate. Signal detection analyses provided further support for this notion, showing that neither sensitivity nor response bias differed between the two- and four-face groups. Thus, cuing effects existed for faces encoded in pairs and groups of four.

\section{ORIENTING CUING EFFECTS}

Finally, we attempted to place the cued face recognition effects in context by comparing them to traditional, non-cued recognition memory. This we referred to as orienting the effects of cuing. Given that context reduced false-alarm rates (thus increasing recognition accuracy), we wondered if this is a result of the benefit of providing context (i.e., single faces have no context), or whether it is beneficial only as a means to reduce the 
disadvantage for having encoded more faces at once. Therefore we compared the no-cue trials across the three face encoding groups. Results are mixed. In Experiment 4, there were no differences in hits or false alarms for test faces, regardless of the number of faces presented at encoding. Contrary to Experiment 4, false-alarm rates in Experiment 5 statistically differed between groups on the no-cue trials such that false-alarm rates in the four-face condition were significantly higher than in the single-face condition, and falsealarm rates in the two-face condition fell between the two. However, Bayesian analyses provided weak support for this difference.

There are a few reasons why we may have obtained these weak differences between groups, such as varying cue types at testing (i.e., the single-face condition was only ever tested with no cues), or the variance of the total number of faces studied at encoding (i.e., the single face condition studied 36 faces, while the four-face condition studied 36 faces multiplied by 4 blocks). However, we would have expected differences between groups to arise in Experiment 4, which they did not. Perhaps the most feasible methodological cause for this difference would be that our associative encoding manipulation affected the allocation of attention when participants were encouraged to make judgment tasks. For example, it is possible that participants split their encoding time evenly between pairs of faces, but not groups of four faces. Yet, we would expect such differences in encoding to impact the hit rate, which they did not. What we can say is that something about encoding groups of four faces in our experiment was more difficult for participants than encoding single faces or pairs of faces, even when they encoded the same number of faces in a study-test block. However, this difference was also minimal. Therefore, when participants were given contextual cues, the benefits of cuing appear to be a compensation for what was a slightly more difficult task of seeing multiple faces at once.

\section{LIMITATIONS}

It is important to consider our results within the limitation that we used identical images at study and test. Some researchers justifiably argue that this ignores the natural variability across representations of a face (see Burton, 2013). Because recognition for unfamiliar faces is fragile to even minute deviations, including, lighting, hair-style, image hue, expression, and focal point of the camera (e.g., Jenkins, White, Van Montfort, \& Burton, 2011), it is likely that using the same photographs at encoding and recognition results in an easier task and, thus, overestimates eyewitness memory performance for person recognition (Bruce, 1982). Although it is true that this method does not provide the most realistic test of cued face recognition, there is little reason to assume this approach undermines the results presented here. Not only do we find similar patterns of recognition between faces and non-face stimuli (e.g., Nortje, Tredoux, \& Vredeveldt, 2017), research in 
face recognition using the same images at encoding and recognition has produced similar patterns of results to those tested in the eyewitness paradigm where the faces are always different at encoding and recognition (i.e., confidence-accuracy calibration: Sauer, Brewer, \& Weber, 2008; Sauer, Brewer, Zweck, \& Weber, 2010, Weber \& Brewer, 2004). Interestingly, our results are also in line with Wells and Pozzulo's (2006) test of the novel two-person serial lineup against the traditional simultaneous and sequential lineup procedures: while there were no differences in lineup procedure for accurate identification decisions for target-present lineups, the two-person serial lineup consistently produced fewer false-identifications in target-absent lineups. Although our task may not generalize fully to the more complex real world task of face recognition, it is evident that it provides a basis for testing associative memory of faces.

\section{CONCLUSION}

Across two experiments, we sought to replicate previous work that has demonstrated the benefits of cued face recognition for paired faces, to understand those findings in comparison to straightforward single-face recognition, and to extend those findings to situations in which participants study more than two faces. We failed to replicate previous research in cued face recognition with face pairs in the sense that the hit rate for true recognition did not increase when correct cues were available. However, we found that any cue (correct or incorrect) could reduce the false-alarm rate and that these effects extend to those studying groups of four faces, as long as associative encoding was engaged. Furthermore, we demonstrated that cuing likely compensates for the more-difficult task of studying multiple faces at once (vs. single faces), even when divided attention and memory load are controlled for, and that this occurs by reducing response bias. However, the inconsistent effects reported between Experiments 4 and 5 warrant caution in the application of the findings in real world contexts (e.g., Wells \& Pozzulo, 2006). In order to apply cued face recognition techniques to eyewitness identification procedures, future work should extend such findings in experimental settings that are incrementally closer to the eyewitness identification context, such as using video-to-picture methodology, and using fewer trials.

Our results confirm the utility of using other faces as contextual cues to enhance recognition accuracy. However, our work suggests that accuracy is enhanced (1) by a decrease in false recognition rather than an increase in true recognition (decreased falsealarm rate), and (2) a result of a shift in response bias rather than memorial enhancement. This research was the first replication of original cued face recognition findings using contemporary methodological procedures (i.e., randomization of face groups and left-right 
placement), as well as on extending such cued effects to situations in which there are more target faces presented at the same time. 
CHAPTER 5 
CHAPTER 5

\section{General Discussion}

This chapter is based in part on the following publication:

Tupper, N., Sauerland, M., Hope, L., \& Merckelbach, H.M.J. (2015). Seeing and believing: Common courtroom myths in eyewitness memory. In Mind, 28, 1-6. (see: http://www.in-mind.org/article/seeing-and-believing-common-courtroom-mythsin-eyewitness-memory) 


\section{AIMS OF THE THESIS}

Eyewitness testimony is an abundant and important source of evidence in criminal law. Variants of this evidence include eyewitness statements, perpetrator descriptions, and identification of suspects from surveillance footage, from books of mugshots, and from lineups. This programme of doctoral research focused on this last form of evidencerecognition and identification of unfamiliar faces - in the context of multiple perpetrator crime. In particular, the research focused on two factors: the associations between multiple decisions and the associations between memories for multiple faces. Across five empirical studies and one exploratory survey, this thesis reviewed police practice in three EU countries in the context of multiple suspect identification (Chapter 2: Police Survey), tested the independence of multiple identification decisions made successively (Chapter 3: Experiments 1, 2 and 3), and examined the purported utility of contextual face cues for recognizing the faces of multiple perpetrators (Chapter 4: Experiments 4 and 5). A key aim of the research was to examine concerns in current eyewitness identification procedures for multiple perpetrators. In particular, to explore those factors relevant to previously unsuccessful attempts to create novel identification procedures adapted to the context of multiple perpetrator crimes. In this discussion, an overview of the key findings is presented, followed by theoretical implications for memory and decision-making, and practical implications for researchers in the subject of multiple perpetrator identification and for police in the field. This is followed by an examination of the limitations of the research presented here, and suggestions for future research.

\section{SUMMARY OF FINDINGS}

Police survey. The thesis started with a survey-based review of police practices in three EU countries: Sweden (SE), Belgium (BE), and the Netherlands (NL). Previous police surveys have not looked into the issue of multiple perpetrator identifications (the one known exception being Hobson et al., 2012). Thus, this survey was conducted to obtain police perceptions of prevalence and characteristics of multiple perpetrator crimes and the eyewitnesses to those crimes, and to discern how identification procedures for multiple suspects are conducted. Results highlighted the practices that are similar between countries as a result of established regulations (i.e., using mostly photographic, sequential lineups), and practices that vary between and within countries in the absence of specific protocols to follow (i.e., whether to put multiple suspects in the same or in different lineups). For the purpose of this thesis, three findings from this survey are particularly relevant. First, there are very few regulations concerning multiple perpetrator crimes, both for a country that requires certification for officers to conduct identification tests (NL), and for the countries that do not (SE, BE). Second, in the absence of such rules, officers appear to apply a set of 
similar extensions of procedures they use for single-perpetrator lineups. For example, most officers avoided telling eyewitnesses for which of the multiple perpetrators the suspect lineup was created, even though there is no rule that expressly forbids this practice. Further, while most officers in the three countries created separate lineups for each suspect of the multiple perpetrator crime, a percentage instead favoured placing all suspects in the same lineup. Third, reported practices make it clear that the association between multiple, separate decisions (i.e., making multiple identification decisions), and the association between the multiple memories of faces (i.e., multiple suspects corresponding to different perpetrators in the same lineup) are critical avenues for future research.

Sequential dependencies. The third chapter of the thesis focused on the association between multiple, separate recognition decisions. Previous work in recognition memory has demonstrated that decisions, although ostensibly separate, are not independent (Malmberg \& Annis, 2012). In Experiments 1 and 2, we tested the relationship between previous identification decisions and current choosing behavior in the context of the multiple showup identification decisions for a multiple perpetrator crime using a mockeyewitness paradigm. Across experiments, evidence for sequential dependencies for choosing behavior was inconsistent: We could sometimes predict choosing from previous choosing patterns and sometimes not. Experiment 3 examined whether methodological differences between the recognition and eyewitness paradigms used in previous research on sequential dependencies could account for the inconsistent findings obtained in Experiments 1 and 2. Experiment 3 therefore sought to replicate previous recognition research in sequential dependencies using word and landscape stimuli, to extend these effects to face stimuli, and to examine whether the strength of these sequential dependencies changed as a result of the number of test trials (i.e., beginning vs. middle of experiment). Sequential dependencies were detected in recognition decisions over many trials, including recognition of faces: Overall, the probability of a "yes" response on the current recognition trial increased if the response on the previous recognition trial was also "yes" (vs. "no"). Although the expected dependencies arose across the many trials, these effects did not mean that the outcome of any individual trial could be reliably predicted by the previous one. Given that sequential dependencies did not notably impact observed choosing behavior for any individual trial, it is unlikely sequential dependencies would have a substantial impact on applied identification procedures or eyewitness choosing patterns across multiple lineups decisions.

Cued face memory. The fourth chapter of this thesis focused on the association between memories for multiple faces seen at the same time. Previous research suggested that providing a cue face increases hits for its previously studied pair (i.e., target face). Experiments 4 and 5 examined the purported utility of associative memory for recognizing 
the faces of multiple perpetrators, investigated the mechanisms underlying those effects, and sought to determine whether such effects could include more than two faces. These experiments attempted to replicate previous experiments that paired to-be-encoded faces, and then tested whether recognition performance was enhanced when test faces were paired with the correct face cue (the second face of the encoded pair), an incorrect face cue (a never-before-seen face), or no cue was presented next to the test face. We compared this replication condition with two new conditions with fewer faces (single faces) and more faces (groups of four faces) presented during encoding. The correct recognition of previously-studied faces (hits) was not affected by face cuing. However, face cuing of any kind (correct or incorrect cuing) appeared to provide a protective buffer to reduce the rate of false recognition of never-before-seen faces (false alarms), regardless of whether there were two faces or four faces at encoding. This appeared to be a result of reduced response bias.

\section{THEORETICAL IMPLICATIONS}

Sequential dependencies arise for faces. This research was the first to test face recognition decisions for sequential dependencies, which have been consistently reported for a wide variety of tasks, including perception of sound volume, categorization of facial emotion expressions, wine taste perception, and decisions by asylum judges (Chen, Moskowitz, \& Shue, 2016). Holland, \& Lockhead, 1968; Hsu \& Yang, 2013; Schifferstein \& Frijters, 1992). Experiment 3 showed for the first time that sequential dependencies can arise for recognition of images of faces. Although recognition accuracy for faces was lower overall compared with words and landscape images, sequential dependencies arose in similar patterns as for other stimuli. This is particularly interesting because there are many instances exposing how we encode faces differently compared with other stimuli. In contrast to encoding of objects, for example, it is difficult (if not impossible) for us to encode multiple faces at the same time. Rather, it is likely that we suppress the processing of one face in favor of devoting our attentional resources on the other face (Bindemann, Burton, \& Jenkins, 2005; Bindemann, Jenkins, \& Burton, 2007). Yet despite such differences in encoding, faces are subject to the same recognition dependency patterns over many recognition decisions. This is perhaps less surprising when we consider that most recent models explaining the patterns of sequential dependencies posit interference at the level of perception during testing, not encoding (Brown, Marley, Donkin, \& Heathcote, 2008; Matthews \& Stewart, 2009; Stewart, Brown, \& Chater, 2005). Thus, it is unlikely to matter what type of item was encoded or how it was encoded, as long as the item was encoded at all. And if such models are correct, cognitive processes would be affected as memory is accessed during testing, whether that memory is of items, landscapes, or faces. In summary, 
while it is more difficult to remember faces, it is one more category of stimuli for which sequential dependencies arise across multiple decisions. Therefore, we could hypothesize that other tasks to measure sequential dependency patterns (e.g., judgment of frequency tasks, categorization tasks) would likely produce similar results when using faces.

This research was also the first to examine sequential dependencies in the field of eyewitness identification, specifically for multiple perpetrator crimes. However, at the time of writing, there has been one other recent study that considers the effect of making multiple lineup decisions, although not within the theoretical framework of sequential effects (Mansour, Beaudry, \& Lindsay, 2017). Mansour and colleagues asked whether multiple-trial experiments are appropriate for eyewitness research; in particular they focused on whether accuracy, choosing, and confidence rates changed over the course of eyewitness trials. Participants watched 24 videos and made 24 lineup identification decisions on target-present and target-absent trials. They found that the number of trials had no effect or a trivial effect on accuracy, choosing, or confidence. This is in line with our results from Experiments 1 and 2 on making multiple showup identification decisions, for which we found little capacity to predict choosing behavior based on previous choosing. Furthermore, in Experiment 3, we found sequential effects for face recognition overall, but still could not predict choosing behavior on individual trials. Combined, these results suggest that sequential effects in themselves are not likely to greatly impact making multiple identification decisions in a row in an eyewitness context.

That patterns of choosing behavior do not change within a set of three identification trials serves to highlight the critical importance of understanding initial expectations of eyewitnesses that impact the criterion they will use for the multiple identification trials. In the literature on single suspect lineups, for example, researchers consistently find that certain instructions can alter identification accuracy for better or for worse. For example, telling an eyewitness that the perpetrator may or may not be in the lineup increases correct rejections of innocent suspects in target-absent lineups without damaging accurate identifications of actual perpetrators in target-present lineups (unbiased instructions; see meta-analysis by Steblay, 1997). Another example is that reminding eyewitnesses the perpetrator's appearance may have changed between the event and the lineup (i.e., the perpetrator may have cut their hair, grown facial hair, or gained weight), increases inaccurate identifications of innocent suspects but not accurate identifications of perpetrators (appearance change instruction; Charman \& Wells, 2007). These instructions alter eyewitness expectations of the task and therefore the criteria used to choose or reject from a lineup to increase the likelihood of being accurate. Unbiased instructions cause eyewitnesses to adopt a more conservative response strategy that requires more evidence or more certainty for choosing someone from the lineup. Meanwhile, appearance change 
instructions relax that response strategy, allowing for more flexibility if a person does not exactly match the memory of the perpetrator. Similarly, it is critical to understand what expectations eyewitnesses carry into multiple suspect lineups. For example, people appear to naturally engage in probability matching in order to maximize optimal responding for multiple decisions (see Vulkan, 2000), meaning they use an expectation of base rates to inform current decisions. An example of this is when students, knowing that their teachers tend to vary correct responses on multiple-choice tests (i.e., answer options A to D), become suspicious after circling too many As. Response patterns can be altered from the outset by providing accurate information (i.e., half of the trials will be new faces and half will be old faces) or inaccurate information (i.e., only five of 100 trials will have an old face). Similarly, eyewitnesses to multiple perpetrator crimes may hold such expectations when confronted with multiple suspect lineups. For example, witnesses in the U.K. who attempt to pick multiple perpetrators from the same single-suspect lineup (Hobson, Wilcock, \& Valentine, 2012) hold accurate expectations that multiple suspects have been apprehended but inaccurate expectations that those multiple suspects are in the same lineup. It is important to explore the boundaries of these expectations for multiple suspect lineups and the resulting choosing behaviors for multiple suspect identification procedures.

Faces as contextual cues for recognition may shift response bias. This research was the first to test the effects of cued face recognition against non-cued face recognition by comparing the traditional paired face condition and the four-face condition with the single-face condition. The aim of this experiment was to create a baseline comparison of accuracy rates when participants studied a set of individual faces, pairs of faces, or groups of four faces and were tested on their memory for those faces without cues. If these accuracy rates are equivalent, then any increase in accuracy as a result of cuing would demonstrate how context enhances memory beyond typical rates of remembering and any decrease in accuracy would demonstrate the risks of using context. If these rates are not equivalent, namely that accuracy for participants studying single-faces are higher than for participants studying groups of two or four faces, then any benefit of cuing reflects a compensation for reduced accuracy. We found that the false-alarm rate increased as the number of faces to be studied increased, but the hit rate did not. Thus, while there was no effect on true memory for studied faces, it appears that studying multiple faces led to inaccurately endorsing the recognition of never-before-seen faces more often than studying individual faces. Our research therefore suggests that cuing may help to compensate for the multiple face disadvantage.

This research was also the first to test cued recognition memory when more than two faces were present at encoding. Typically we would expect that a correct cue at test enhances memory for previously studied faces and therefore increases accuracy rates. But 
there were several reasons to suspect that cuing with four-face stimuli may not be as effective for memory as cuing with two-face stimuli. First, an increased cognitive load associated with having more faces (e.g., four vs. two) might reduce the initial memory contextual cues could support. Second, it was possible that the test conditions with cues represented only a partial reinstatement of context for the four-face group, but a full reinstatement of context for the two-face group, would not be strong enough to support memory. Third, the potential to create associations between some, but not all of the faces in the four-face group, could mean that the face chosen by experimenters to be a contextual cue during testing may not be the one that was most strongly associated with the target at encoding. In contrast to the concerns listed above, our results showed that cuing was equally effective for both the two-face and four-face groups in reducing false alarms, meaning that participants were more accurate in responding that a never-before-seen face was, in fact, "new". This suggests that cuing effects may be able to be extended to more complex scenarios. This finding is particularly important to consider given that many crimes are committed by more than two perpetrators, and therefore research should take larger groups of stimuli (i.e., faces) into consideration in constructing and exploring theoretical models for memory and decision-making.

That contextual face-cues work equally well for the two-face and four-face groups is less surprising when we consider that accuracy rates improved as a result of reduced response bias, rather than enhanced memory. Thus, participants were not better at remembering the previously-seen faces, but were more cautious in deciding whether or not to endorse that they had previously seen a face. While shifts in response bias have been found in the contextual cuing literature, it is usually accompanied by an improvement in memory as well (Davies, 1988). However, it is also important to note that in the experiments reported in this thesis, any form of contextual cue improved accuracy: not only did the accurate face-cues at test decrease the rate of false alarms, but so did the inaccurate facecues. This means that presenting cues of previously-studied faces and presenting cues of never-before-seen faces both improved participants capacity to accurately respond that a face was "new". These results challenge the typical findings in cued recognition, where accuracy rates are higher for accurate cues than for inaccurate cues. It is difficult to explain why the presence of an inaccurate cue would lead the response bias to become less liberal, similar to the presence of an accurate cue. It may be that cuing, regardless of the veracity of the cue, induced suspicion and thus participants to remember to reject in light of uncertainty, or that cuing increased the strength of evidence (i.e., the sense of familiarity or availability of memory) participants require for a positive response. However, these explanations need to be addressed in future research on contextual cuing. 
Unexpected findings. The presented experiments in sequential dependencies and cued face recognition were similar in that they produced unexpected results. The predicted effects did not show up or, if they did, they presented in unexpected ways: The robust effects of sequential dependencies did not arise in the eyewitness paradigm for multiple identification decisions and the cued face recognition effects did not arise in the first attempt. While the cued recognition effects did present in the second attempt when a manipulation was added to increase the strength of associative encoding, even these effects were contrary to expectations. Unlike previous work with cued face recognition, contextual face cues in the presented experiment affected false-alarm rather than hit rates. These surprising results suggest a number of moderators may be at play, including strength of associative encoding. Current theoretical perspectives need to be expanded through research better elucidating the boundary conditions and moderators of these core effects.

\section{A CRITICAL INVESTIGATION OF THE STATE OF MULTIPLE PERPETRATOR RESEARCH}

The research presented in this thesis is in many ways an attempt to act as counterweight to common sense. Psychology is often believed by laypeople to be common sense dressed as science. In fact, the radio talk show host, Dennis Prager, and the journalist known for contributions to Scientific American, the New York Times, and Newsweek, John Horgan, both agree that we should be suspicious of the extent to which scientific theories contradict our common sense (Lilienfeld, Lynn, Ruscio, \& Beyerstein, 2010). In cases when judges bar eyewitness memory experts from testifying, they often rule that the psychologist cannot offer information outside of general common sense (e.g., State v. Coley, 2000; U.S. v. Libby, 2006). While common sense is a useful heuristic in daily life, it does not always lead to accurate beliefs (see Lilienfeld et al., 2010 for a list of sources for myths in psychology). And while holding inaccurate common-sense beliefs is not desirable, there are rarely dire consequences in daily life for holding them. What does it matter, for example, if people believe that reading by a dim light ruins eyesight despite no empirical evidence; or if they think that vision involves emitting rays of energy at some point before or during the perception process? And while insomniacs may count sheep to fall asleep, they are not at risk for anything more serious than wasting time (Lilienfeld et al., 2010). However, the justice system is a field where common-sense beliefs are applied by police officers, lawyers, judges, and jurors throughout investigations and trials (e.g., Chapter 2, this dissertation; Hobson et al., 2012; Benton, Ross, Bradshaw, Thomas, \& Bradshaw, 2006). All of these legal decision-makers holding common-sense beliefs about eyewitness memory have the capacity to make life-changing decisions for victims, witnesses, suspects, and perpetrators. Unfortunately, many of these beliefs are in conflict with empirical evidence. For example, 
only $41 \%$ of surveyed jury-eligible Americans believe that lineup instructions can impact the accuracy of an identification and only $50 \%$ know that eyewitness confidence in that identification is highly susceptible to outside influences (compared to $98 \%$ and $95 \%$ of memory experts, respectively; Benton et al., 2006). We find similar disagreements between lay people and experts regarding how the presence of a weapon impacts encoding and how race plays a role in identification (Houston, Hope, Memon, \& Read, 2013). This is indeed because there are many aspects of memory that are not common sense.

Although it is important to acknowledge that some research seeks to isolate the underlying mechanisms of multiple face identification and recognition disadvantageincluding determining whether eyewitnesses mix up face features between the perpetrators (i.e., Megreya \& Bindemann, 2012), or whether the disadvantage stems from memorial capacity, or number of comparisons (Bindemann, Sanford, Gillatt, \& Megreya, 2012) - most research in multiple perpetrator identification has tested common-sense solutions to identification procedures. Lineups for multiple perpetrators have been presented in different orders and with new instructions (i.e., Hobson \& Wilcock, 2011), they have been tested using techniques attempting to control for relative vs. absolute judgments (i.e., Dempsey \& Pozzulo, 2008; 2013), and combined lineups to present suspects and fillers for both perpetrators at once (i.e., Wells \& Pozzulo, 2006). However, this research tests common-sense solutions for a problem that is not yet well understood. Therefore, results for these solutions do not provide answers as to why they benefit identification accuracy or, more often, why they fail to do so. The a-theoretical nature of such investigations limits the generality of their findings, and such results are therefore difficult to build upon in future research.

Meanwhile, the most useful research explores the problem of the multiple face disadvantage and tests which variables do or do not contribute to it. In doing so, this research systematically works its way through a list of variables to find those factors we should consider when creating novel lineup techniques or protocols. For example, the current thesis tested for sequential dependencies to determine whether decision-making on multiple lineups is impacted simply because of the task of making multiple decisions. Given that these sequential dependencies for recognition did not translate to predictable observable behavior, it is unfruitful for the field of eyewitness identification to find solutions for a variable that is not problematic in the first place. An example of a common-sense solution would have been to provide instructions that specifically addressed the idea that the multiple lineups are not connected; if these instructions improved identification accuracy, we would not have known if they were compensating for sequential dependencies or, for example, moving criterion-placement by making respondents more conservative overall; if the instructions were unsuccessful in improving accuracy, we would 
not have known if they failed to appropriately address sequential dependencies or if sequential dependencies were not an issue that needed to be addressed. By contrast, once we identify and understand the variables that contribute to the multiple face disadvantage, we can test for solutions that might compensate the disadvantages posed by these variables. For example, in this thesis I chose to explore cued face recognition in order to address the previously-tested variable of increased memorial demand (Bindemann et al., 2012). Based on the results provided in Chapter 4, it seems that providing any context of the other suspect may help recognition accuracy. While this work requires replication and further tests, particularly to elucidate why providing any context inhibits false alarms but does not improve hit rates, it is a solution that may be worth the time and resources to pursue.

The fact that we did not replicate cuing effects on hit rates serves as a caution to research building on older studies that have not been recently replicated or more thoroughly explored. For example, Dempsey (2014), and Wells and Pozzulo (2006) have applied cuing theory to create two different, novel identification procedures to enhance memory for multiple perpetrators. One presented the face of only the second suspect next to the entire lineup for the first suspect (Dempsey, 2014); the other presented both lineups for both suspects at the same time, although in a sequential presentation style (Wells \& Pozzulo, 2006). In neither case did cuing improve identification accuracy for the eyewitnesses, and results did not provide clues for why. However, there are two important considerations in mining older theoretical work for newer applied solutions. First, cued face recognition is one example of research that is conducted for one purpose and is later used for another. In early manuscripts of cued face recognition, there is no indication that the authors anticipated that their results would be applied in a practical context such as eyewitness identification. The experiments were designed to test associative memory, not to provide a solution for reduced eyewitness memory in the context of multiple perpetrators. This is certainly not to say that solutions for new problems cannot stem from older research. Rather, that it is important to consider how methodological choices pertinent to the original aims might affect the generalizability of findings to new domains.

For example, in the Watkins and colleagues (1976) and Winograd and RiversBulkley's (1977) experiments, incorrect face cues were created by mis-matching pairs of faces, or having participants circle the pairs of faces that correctly matched. This is an entirely different task compared with separating old faces from new ones. Also, in the typical recognition paradigm, participants view hundreds of faces and are tested on hundreds of trials in order to determine overall trends in memory. Even if the cued recognition effect is successful in such a paradigm, it is possible that the effects are too small to consistently arise when participants are only memorizing a few faces and only 
tested in a few trials. While the experiments presented in this thesis were designed to explore the underlying mechanisms of the cued face recognition effect, and to extend this to situations beyond pairs of faces, this research line still requires several intermediary steps before we can determine whether it has practical value as a solution to multiple perpetrator lineups or to explain the results when we do apply those solutions. For example, does this effect replicate when using videos of targets instead of photographs during encoding? Does the enhancing effect of context cues for false alarms consistently arise when participants encode and are tested on very few faces? These are questions that need to be answered before applying them to lineup solutions.

Second, the original experiments on cued recognition with face cues were conducted in the 1970s, and have only been revisited in the last decade as the field of multiple perpetrator identification and multiple person recognition have gained some interest. However, the field of psychology has since experienced a methodological and statistical revolution in the face of a replication crisis (e.g., Simmons, Nelson, \& Simonsohn, 2011). In other words, we now understand that some classic effects in psychology may be false positive findings - artifacts of an accumulation of issues, such as hiding null findings in the file drawer, reporting only significant results, conducting experiments with small sample sizes, failing to protect against experimenter influence, and failing to adhere to stopping-rules in data collection. Because research cannot take for granted that phenomena will replicate and generalize, experimenters need to be more programmatic in replicating key phenomena, and then systematically extending these effects across stimulus types or moderating influences. This is a lesson learned in this dissertation, first in Chapter 3 , when it became clear it was necessary to step into the recognition paradigm to understand the boundaries of effects of sequential dependencies (i.e., whether they would arise for face stimuli and to what extent), and again in Chapter 4, when the expected cued recognition effects did not replicate. In both cases, it became particularly important to replicate and understand original effects along with extensions of those effects, and both lines of research require even further investigation.

Police officials and legal decision makers are the end-users of accumulated research on multiple perpetrator identifications, and therefore particularly important to consider when it comes to the applied value of such research. To date, police have been using common-sense adaptations to create and administer multiple perpetrator identification procedures. These decisions are sometimes at odds with each other, such as many officers' decisions to create separate lineups for the multiple suspects, or some officer's decisions to create one lineup with multiple suspects. On the one hand, putting multiple suspects in the lineup goes against the common-sense extension of the gold standard for single-suspect lineups, a standard that is in place because it is considered 
imperative to decrease the probability of misidentifying an innocent suspect. On the other hand, it is not immediately obvious by common sense that such rules should apply when multiple suspects relate to different people. These are reasonable adaptations given that there are unlikely to be guidelines specific to multiple perpetrator crimes and that there is little understanding of the difficulties that might arise for either lineup technique.

Unfortunately, the research field is not yet ready to provide practical advice to police officers or policy makers regarding the best methods for testing identification for multiple perpetrator crimes. For one, it appears that there is no obvious harm in placing suspects for the different perpetrators in separate lineups, since sequential dependencies have no substantial effect when eyewitnesses make multiple decisions. Nevertheless, sequential dependencies are not the only way decisions can be linked. For example, probability matching strategies lead participants to respond according to their beliefs about base rates for all possible responses (e.g., Rotello, Macmillan, Hicks, \& Hautus, 2006; Van Zandt, 2000; Vulkan, 2000). A mock eyewitness participant in a lab, for example, might incorrectly assume that an experimenter will not present all of the perpetrators in a series of lineups. This may lead the participant to not choose from all the lineups even when the perpetrators are present. Alternatively, an actual eyewitness may expect that the police have caught all of the perpetrators and therefore choose from a lineup whether the actual perpetrator is present or not. Thus, while our results suggest that sequential dependencies are not problematic in the context of lineup decisions for multiple perpetrator crimes, there are more factors in making multiple decisions to explore. Meanwhile, our results for cued face recognition suggest it may be advantageous to have the multiple suspects related to different perpetrators in the same lineup, since context may help to reduce the possibility of falsely identifying an innocent suspect. However, our results would need to both be replicated in a recognition paradigm and subsequently tested in the eyewitness identification paradigm while controlling that one of the suspects might get picked by chance before we can recommend such an action. Given the dearth of understanding in eyewitness memory and identification for multiple perpetrators, it would be irresponsible to supply advice to police as to the correct protocols. At the moment, we can only provide a greater understanding surrounding the protocol decisions already being made.

\section{METHODOLOGICAL CONSIDERATIONS AND FUTURE RESEARCH}

This thesis takes a multifaceted approach to consider eyewitness recognition and identification for multiple perpetrator crimes with studies that investigate police practice, memory, and decision-making. This approach allows us to initiate research in a wide range of topics within identification for multiple perpetrators. For example, we examined eyewitness decision making for showup identifications and recognition decisions. Although 
we conclude that sequential dependencies themselves are not significantly problematic in the field of eyewitness identification, there are other ways in which decisions could be linked. Future research should explore whether expectations play a role, such as eyewitness expectations for police competence, and whether suspicion regarding that competence can moderate that effect. For example, the post-identification feedback effect is a robust effect in which feedback from an experimenter or authority figure artificially increases confidence for an identification decision, regardless of the actual accuracy of the eyewitness (Steblay, Wells, \& Douglass, 2014). However, inducing suspicion about the trustworthiness of the source of feedback helps to eliminate the effects of feedback (Neuschatz et al., 2007). Specifically, when an experimenter escorting the participant to a location change revealed that the study was being funded by the Tennessee District Attorney's office in an attempt to prove the accuracy of eyewitness identifications, and the experimenter was telling everyone that they had picked the perpetrator from the lineup, participants did not show the typical confidence inflation from the positive feedback. In such a case, if an eyewitness believes police to be competent, and then recognize a perpetrator in the first lineup, do they carry those expectations forward to assume the next lineups will also have a perpetrator? Does this change if eyewitnesses are induced to be suspicious?

Another example for which decisions might be linked is when more than one suspect is presented in the lineup. In such a case, eyewitnesses must make decisions for multiple suspects at the same time. Meanwhile, it is important to consider how sequential dependencies may impact applied jobs that involve making a large number of recognition decisions. For example, officials at football matches on the lookout for banned hooligans, or border control officers matching people to their passport photos. While all member states of the European Union report violence during football matches, the highest rates of football-related violence have historically been seen in England, Italy, Germany, the Netherlands, and Belgium, with at least $10 \%$ of supporters classified as "violent" (Carnibella et al., 1996). As recent as 2014, the European Commission has supported attempts to coordinate football leagues and security services to prevent or contain violence at these matches (European Commission, 2014). Spotters may be given books of fans that have been banned from the game, and a large number of low- to high-risk fans attending the game to identify. In stadiums of tens of thousands of spectators, these spotters scan the crowd, making a high frequency of rapid recognition decisions. As some regions train specialized teams (i.e., Rotterdam police force) to aid recognition, it may be of interest to understand how sequential dependencies may influence their accuracy across these decisions.

This dissertation also contains experiments designed to examine the link between eyewitness memories by testing cued face recognition. Two studies attempted to replicate and extend previous works on cued face recognition in which a single-face condition was 
compared with the two- and four-face conditions on those trials that did not use a face cue. The single-face condition was considered a control in order to determine baseline differences in recognition accuracy between the three groups. However, participants in both multiple face conditions also encountered other trials that included correct and incorrect cues, and therefore their testing condition differs from that of the single-face condition. This difference was minimal because although it was statistically significant, there was very weak support for this difference from Bayesian analyses. Furthermore, this difference did not arise in the first experiment, where testing conditions were the same. However, given that this difference in no-cue trials between groups did present in Experiment 5, it may be a valuable comparison for participants to be presented with only no-cue trials, thus keeping testing conditions constant. This would be a logical next step for future research in order to understand the benefits of cued recognition. Another future direction for this field is the aforementioned incremental steps to conduct cued recognition research with videos of faces, and with very few trials, and, lastly, within the eyewitness paradigm for cued face research.

One difficulty encountered in Experiment 5 within the cued-recognition paradigm was the need to purposefully encourage participants to create a meaningful connection between the presented faces when studying pairs of faces or groups of four faces. Winograd and Rivers-Bulkeley (1977) called this process 'unitization', which they implemented in their research by asking participants to rate compatibility between couples (male and female target faces) shown on the screen. This would not be an appropriate manipulation to include because of our additional conditions. This was in part because of the stimulus materials (i.e., we used only male faces) and in part because of the difficulty of asking participants to rate romantic compatibility between four targets in the four-face condition. Furthermore, there is no direct proxy for romantic compatibility for only one face in the single-face condition. Thus, in repeating Experiment 4, Experiment 5 included a judgment task aimed to encourage participants to consider the (fabricated) relationship between first impressions of and the memorability of guilty defendants' faces and sentencing length. In this task, each pair or group of faces presented on screen during the study phase was described as presenting perpetrators of a multi-perpetrator crime, who had been convicted by a jury and sentenced to equivalent prison terms. Participants were asked to use their initial impressions of the faces together to estimate how many years the pair or group had been sentenced to prison. This was considered an appropriate manipulation because criminals that commit a crime together are seen as a very closely-linked, also known as highly entitative, group (Lickel et al., 2000). Furthermore, because participants were asked to use their impression of all of the faces in order to estimate a single sentencing length, it was expected this judgment would actively engage in unitized or associative encoding. 
However, without eye-tracking equipment, it is not possible to know for certain that participants attended to all available faces when making this judgment. It is possible, for example, that they only used one of the faces to determine sentence length for the whole group. If this is the case, the faces themselves would not be integrated in memory as associated items and cuing would not be useful to enhance access to memory. This might explain why cuing did not improve participant hit rates, for example. Nevertheless, given that Experiment 5 was a direct replication of Experiment 4 except for the addition of this judgment task, this explanation is difficult to reconcile with the change in false-alarm rates between experiments. It is unclear how a judgment task at encoding would lead to a shift of response bias at test when cues are employed.

Even with a multifaceted approach, this dissertation only touched on a few of the many research opportunities present in the field of multiple perpetrator identification. It is important, for example, to explore factors of the encoding event, such as how attention is distributed among members of a group of perpetrators, how salience of the perpetrator (i.e., central vs. peripheral perpetrators) impacts which members are remembered, and the degree of similarity between perpetrators (e.g., gang members that look alike). Another is to explore those factors at test that are unique to multiple perpetrator recognition and identification, such as whether the multiple suspects should be placed in the same lineup or whether eyewitnesses should be instructed to search for specific suspects (i.e., this lineup is for the man that held the gun). We began tapping into only some questions in decisionmaking, memory, and practice that are relevant to eyewitnesses of multiple perpetrator crimes, but there are more variables at encoding, retention, and recall/recognition that are both unique and inherent to multiple perpetrator crimes.

\section{LIMITATIONS}

As with any research, it is important to understand the implications of the reported results within the context of their methodological limitations. For example, with the exception of the police survey, these experiments sampled from a population of university students with an overrepresentation of women. There are some gender differences that are apparent in face recognition and identification. Specifically, women have been shown to outperform men in face recognition tasks because women more often display an owngender bias: although men and women recognize male faces at the same rate of accuracy, women generally perform better than men in recognizing female faces (Herlitz \& Yonker, 2002; Hill et al., 1995; Lewin \& Herlitz, 2002; vs. Wright \& Sladden, 2003).

In Experiments 1 and 2 of this thesis, there was one female and two male perpetrators present in the mock crime film. Therefore, all participants (324 women, 80 men) were asked to make recognition decisions about one female and two male suspects. 
It therefore is of interest to determine whether gender of participant and target affected showup identification accuracy rates. Accuracy for the two male identification decisions were averaged together to create one total accuracy score for male targets. Because the outcome variable is nominal, and because the sample sizes differed between groups of male and female participants, a traditional repeated-measure ANOVA could not be used. Therefore, a Generalized Estimating Equation (GEE) was employed, with participant gender (female vs. male) as the between-subjects variable and target gender (female vs. male) as the within-subjects variable. The initial analysis included all main effects (participant gender and target gender) and the resulting two-way interaction in the equation. The interaction between participant gender and target gender was not significant, so it was excluded from the analysis to observe the main effects of gender. The main effect of participant gender was also non-significant, leaving only the significant main effect of target gender. The final model for showup identification accuracy therefore only included target-presence as a predictor $b=-0.06$, Wald $\chi^{2}(1)=4.85, p=.028$. This shows that participants, regardless of their own gender, were more accurate in identifying the female target (67\% accuracy) than the male targets (61\% accuracy).

This result is particularly noteworthy because it is contrary to the research that suggests that women outperform men (Herlitz \& Yonker, 2002), that women's superior face recognition comes from an own-gender bias (Lewin \& Herlitz, 2002), and even the opposing research that suggest that both genders display an own-gender bias in face recognition (Wright \& Sladden, 2003). It is unclear what drives this difference in our results. At first glance, our experiments differ from those reported above because they were conducted within the eyewitness identification paradigm rather than the recognition paradigm. However, Palmer, Brewer, and Horry (2013) found an own-gender bias in both the face recognition and eyewitness recognition paradigm. It is possible that because our mock crime video included two male targets and one female target, both male and female participants paid more attention to the female target during encoding, producing a stronger initial memory trace.

Another limitation of the current experiments in face recognition (Experiments 3, 4 and $\mathbf{5}$ ) is that images of faces were used at both encoding and recognition. Unlike in the eyewitness paradigm (Experiments 1 and 2), where participants viewed a video of perpetrators and then were asked to make identification decisions from photographs of suspects, participants in the recognition paradigm studied photographs of individuals and were later tested on the same photographs. Some researchers argue that this methodology is problematic for our understanding of face recognition, because participants may be matching images rather than recognizing faces (see Burton, 2013). The critique is that using images of faces at encoding and recognition is ignoring natural variability across 
representations of a face, meaning that research is measuring the recognition of face images rather than the recognition of people. This is a reasonable assertion: to recognize someone, we should be able to see them from different angles, in different lighting, and know who they are. For example, celebrities like Oprah, Bob Dylan, Robert de Niro, and Hillary Clinton are easily recognizable across photographs at different ages, in different poses, at different angles, with different backgrounds, clothing, facial expressions, and even artistic filters. Recognition for familiar faces, like those of family members, friends, and celebrities, is robust across these temporal and contextual changes. However, recognition for unfamiliar faces is conversely fragile to even minute deviations, including, but by no means limited to, lighting, hair style, image hue, expression, and focal point of the camera (see Burton, 2013; Jenkins, White, Van Montfort, \& Burton, 2011). Thus, using the same photographs at study and test causes researchers to overestimate eyewitness memory performance for person recognition (Bruce, 1982).

This methodological issue is pervasive in research in recognition memory and identification. Certainly there is no evidence that could argue it is advantageous to use the same photographs at encoding and recognition. However, this decision is typically one of availability of materials. For the current thesis, the large numbers of face images required to test the hypotheses mitigated against the compilation of a new face database. Further, given differences in available databases that prevent combining faces from different sources (i.e., decisions regarding stimulus, cropping, background, camera angle and distance; equipment such as lighting, cameras, and lenses), it was difficult to obtain the number of faces needed for such experiments (i.e., more than 250 photos in each Experiments 3, 4 and 5) when decisions to control other influencing variables (i.e., gender, age, race) further restrict options for faces. The current approach is used as a starting point, but this research should certainly expand to include replication with such realistic variations.

\section{CONCLUSION}

In five experiments and one survey, this thesis examined underlying issues in memory and decision-making that impact eyewitness identification in the context of multiple perpetrator crimes. This thesis explored a range of topics related to the recognition and identification of multiple perpetrators in three areas: practice, memory, and decisionmaking. A survey of police methods provided an initial picture of how police officers approach identification procedures for multiple perpetrator crimes, how lineups are constructed and presented, what type of instructions are given, and problems they experience in conducting such procedures. Responses made clear that there are few regulations for identification procedures specifically for multiple perpetrator crimes and 
that police vary in their decisions on how to create and administer these lineups, decisions that ideally should be tested and advised upon by empirical research. The presented research in sequential dependencies for identification decisions demonstrated that there is little concern that the integrity of identification and recognition decisions is impacted by making the multiple decisions in a row. Lastly, the presented research showed that cued face recognition may be a useful technique in the recognition of multiple perpetrators, and a promising avenue for future research.

\section{A FINAL NOTE ON EYEWITNESS EVIDENCE}

Cases like Alan Crotzer's presented at the beginning of this dissertation highlight the dilemmas inherent in eyewitness evidence. Our unavoidable reliance on eyewitness evidence is pitted against the dangerous consequences of human error. Given research demonstrating the poor accuracy of eyewitnesses making identification decisions, and based on media accounts detailing the pitfalls of eyewitness memory, it would seem easy to assume that eyewitness evidence will be entirely replaced by the hard evidence of blood splatters and DNA traces. Yet eyewitness evidence is here to stay. Although TV shows like CSI suggest otherwise, "hard evidence" is only available in a minority of criminal cases. Indeed, most cases of exonerated innocents were able to be proven innocent because they had been convicted of murder or sexual assault - the subset of crimes that often do leave behind testable biological traces (Wells, Memon, \& Penrod, 2006). But even in the fraction of crimes where police can successfully link evidence like fingerprints and DNA to a suspect, the testimony of an eyewitness is still needed to connect that evidence to the people of the heist. Are fingerprints at the scene of a bank robbery because they are a local client of the bank or because they were involved in the crime? In other words, even in the minority of cases where technical evidence does exist, that evidence requires interpretation, and witnesses play a vital role in that interpretation. Furthermore, science does not argue that we should exclude eyewitness evidence in court. The science does argue, however, that eyewitness evidence always exists within the context of a number of influential factors - all of which deserve scrutiny throughout all phases of an investigation and trial. In light of the prevailing misconceptions regarding memory, experts seek to increase sensitization among legal professionals to variables that impact eyewitness memory, allowing them to obtain more accurate evidence on the one hand, and to appropriately evaluate the credibility of the eyewitness and the reliability of the evidence on the other (Benton et al., 2006; Cutler, Dexter, \& Penrod, 1989; Kassin, Tubb, Hosch, \& Memon, 2001; Leippe, 1995; Wise, Pawlenko, Safer \& Meyer, 2008). This research aims to add to the body of literature on multiple person recognition and identification decisions so that we can provide valuable information to elicit and assess eyewitness evidence in the context of multiple perpetrator 
crimes. Failure to do this guarantees more regrettable cases like Alan Crotzer's, where innocent suspects of multiple perpetrator crimes are left vulnerable to wrongful conviction and actual perpetrators are free to continue offending. 


\section{REFERENCES}

van Amelsvoort, A. (2013). Handleiding confrontatie [Manual of eyewitness identification procedures] (9th ed.). Amsterdam: Stapel \& De Koning.

Annis, J., \& Malmberg, K. J. (2013). A model of positive sequential dependencies in judgments of frequency. Journal of Mathematical Psychology, 57, 225-236. doi:10.1016/j.jmp.2013.06.006

Bentin, S., Allison, T., Puce, A., Perez, E., \& McCarthy, G. (1996). Electrophysiological studies of face perception in humans. Journal of Cognitive Neuroscience, 8, 551-565. doi:_10.1162/jocn.1996.8.6.551

Benton, T. R., Ross, D. F., Bradshaw, E., Thomas, W. N., \& Bradshaw, G. S. (2006). Eyewitness memory is still not common sense: Comparing jurors, judges and law enforcement to eyewitness experts. Applied Cognitive Psychology, 20, 115-129. doi:10.1002/acp.1171

Bindemann, M., Burton, A. M., \& Jenkins, R. (2005). Capacity limits for face processing. Cognition, 98, $177-197$. doi:10.1016/j.cognition.2004.11.004

Bindemann, M., Jenkins, R., \& Burton, A. M. (2007). A bottleneck in face identification: Repetition priming from flanker images. Experimental Psychology, 54, 192-201. doi:10.1027/1618-3169.54.3.192

Bindemann, M., Sandford, A., Gillatt, K., Avetisyan, M., \& Megreya, A. M. (2012). Recognising faces seen alone or with others: Why are two heads worse than one? Perception, 41, 415-435, doi:10.1068/p6922

Bjork, R. A., \& Whitten, W. B. (1974). Recency-sensitive retrieval processes in long-term free recall. Cognitive Psychology, 6, 173-189. doi:10.1016/0010-0285(74)90009-7

Boutet, I., \& Chaudhuri, A. (2001). Multistability of overlapped face stimuli is dependent upon orientation. Perception, 30, 743-753. doi:10.1068/p3183

Bower, G. H., \& Karlin, M. B. (1974). Depth of processing pictures of faces and recognition memory. Journal of Experimental Psychology, 103, 751-757. doi:10.1037/h0037190

Brown, S. D., Marley, A. A. J., Donkin, C., \& Heathcote, A. (2008). An integrated model of choices and response times in absolute identification. Psychological Review, 115, 396 - 425. doi:10.1037/0033295X.115.2.396

Bruce, V. (1982). Changing faces: Visual and non-visual coding processes in face recognition. British Journal of Psychology, 73, 105-116.

Burton, A.M. (2013). Why has research in face recognition progressed so slowly? The importance of variability. Quarterly Journal of Experimental Psychology, 66, 1467-1485. doi:10.1080/17470218.2013.800125

Caharel, S., Ramon, M., \& Rossion, B. (2014). Face familiarity decisions take $200 \mathrm{~ms}$ in the human brain: Electrophysiological evidence from a go/no-go speeded task. Journal of Cognitive Neuroscience, 26, 8195. doi:10.1162/jocn_a_00451

Carnibella, G., Fox, A., Fox, K., McCann, J., Marsh, J., \& Marsh, P. (1996). Football violence in Europe. Unpublished report to the Amsterdam Group. Retrieved from: http://mobile.ihs.mysteria.cz/football_violence.pdf

Charman, S. D., \& Wells, G. L. (2007). Eyewitness lineups: Is the appearance-change instruction a good idea?. Law and Human Behavior, 31, 3-22. doi:10.1007/s10979-006-9006-3

Chen, D. L., Moskowitz, T. J., \& Shue, K. (2016). Decision making under the gambler's fallacy: Evidence from asylum judges, loan officers, and baseball umpires. The Quarterly Journal of Economics, 131, 1181-1242. doi:10.1093/qje/qjw017

Clark, S. E. (2012). Costs and benefits of eyewitness identification reform: Psychological science and public policy. Perspectives on Psychological Science, 7, 238-259. doi:10.1177/1745691612439584

Clifford, B. R., \& Hollin, C. R. (1981). Effects of the type of incident and the number of perpetrators on eyewitness memory. Journal of Applied Psychology, 66, 364-370. doi:10.1037/0021-9010.66.3.364

Cooper, A., \& Smith, E. L. (2011). Homicide trends in the United States, 1980-2008. Washington (District of Columbia): Bureau of Justice Statistics. Retrieved from website: http://www.bjs.gov

Cutler, B. L., Penrod, S. D., \& Dexter, H. R. (1989). The eyewitness, the expert psychologist, and the jury. Law and Human Behavior, 13, 311.

Dauvergne, M., \& Li, G. (2006). Homicide in Canada, 2005. Juristat: Canadian Centre for Justice Statistics, 26(6), 1 26.

Davies, G. D. (1988). Faces and places: Laboratory research on context and face recognition. In G. M. Davies \& D. M. Thomson (Eds.), Memory in context: Context in memory (pp. 35-53). London: Wiley 
Davies, M. (2008-) The Corpus of Contemporary American English (COCA): 520 million words, 1990-present. Available online at http://corpus.byu.edu/coca/.

Davis, J. P., Valentine, T., Memon, A., \& Roberts, A. J. (2015). Identification on the street: A field comparison of police street identifications and video line-ups in England. Psychology, Crime and Law, 21, 9-27. doi:10.1080/1068316X.2014.915322

Deffenbacher, K. A., Bornstein, B. H., McGorty, K., \& Penrod, S. D. (2008). Forgetting the once-seen face: Estimating the strength of an eyewitness's memory representation. Journal of Experimental Psychology: Applied, 14, 139-150. doi:10.1037/1076- 898X.14.2.139.

Dempsey, J. L. (2012). An Investigation of Retrieval Cues as a Method to Improve Eyewitness Identification Accuracy of Multiple Perpetrators (Doctoral dissertation, Carleton University).

Dempsey, J. L., \& Pozzulo, J. D. (2008). Identification accuracy of eyewitnesses for a multiple perpetrator crime: Examining the simultaneous and eliminate lineup procedures. American Journal of Forensic Psychology, 26, 67-81.

Dempsey, J. L., \& Pozzulo, J. D. (2013). Children's identification accuracy of multiple perpetrators: Examining the simultaneous versus elimination line-up. Psychiatry, Psychology and Law, 20, 353-365. doi:10.1080/13218719.2012.679124

Diamond, R., \& Carey, S. (1986). Why faces are and are not special: an effect of expertise. Journal of Experimental Psychology: General, 115, 107-117. doi:10.1037/0096-3445.115.2.107

Di Giorgio, E., Turati, C., Altoè, G., \& Simion, F. (2012). Face detection in complex visual displays: an eye-tracking study with 3-and 6-month-old infants and adults. Journal of Experimental Child Psychology, 113, 66-77. doi:10.1016/j.jecp.2012.04.012

Dunning, D., \& Stern, L. B. (1994). Distinguishing accurate from inaccurate eyewitness identifications via inquiries about decision processes. Journal of Personality and Social Psychology, 67, 818-835.

Egan, D., Pittner, M., \& Goldstein, A. G. (1977). Eyewitness identification: Photographs vs. live models. Law and Human Behavior, 1, 199-206. doi:10.1007/BF01053439

European Commission (2014). Commission decision of 14.10.2014 adopting the Arrangement for Cooperation between the European Commission and the Union of European Football Associations (UEFA). Retrieved from: http://ec.europa.eu/assets/eac/sport/news/2014/docs/uefa2014_en.pdf

Frenda, S. J., Nichols, R. M., \& Loftus, E. F. (2011). Current issues and advances in misinformation research. Current Directions in Psychological Science, 20, 20-23. doi:10.1177/0963721410396620

Frontière belge: Un papi fait fuir deux malfrats qui tentent de kidnapper un livreur (2015, October). Le Vif. Retrieved from http://www.levif.be/actualite/insolite/frontiere-belge-un-papi-fait-fuir-deux-malfratsqui-tentent-de-kidnapper-un-livreur/article-normal-428451.html

Geiselman, R. E., Fisher, R. P., MacKinnon, D. P., \& Holland, H. L. (1986). Enhancement of eyewitness memory with the cognitive interview. The American Journal of Psychology, 99, 385-401. doi:10.2307/1422492

Gigerenzer, G., Hoffrage, U., \& Kleinboelting, H. (1991). Probabilistic mental models: A Brunswikian theory of confidence. Psychological Review, 98, 506-528. doi:10.1037/0033-295X.98.4.506

Herlitz, A., \& Yonker, J. E. (2002). Sex differences in episodic memory: The influence of intelligence. Journal of Clinical and Experimental Neuropsychology, 24, 107-114. doi:10.1076/jcen.24.1.107.970

Hill, R. D., Grut, M., Wahlin, A., Herlitz, A., Winblad, B., \& Backman, L. (1995). Predicting memory performance in optimally healthy very old adults. Journal of Mental Health \& Aging, 1, 55-65.

Hobson, Z. J., \& Wilcock, R. (2011). Eyewitness identification of multiple perpetrators. International Journal of Police Science \& Management, 13, 286-296. doi:10.1350/ijps.2011.13.4.253

Hobson, Z. J., Wilcock, R., \& Valentine, T. (2012). Multiple suspect showing: A survey of police identification officers. Policing, 21, 79-87. doi:10.1093/police/pas021

Holland, M. K., \& Lockhead, G. R. (1968). Sequential effects in absolute judgments of loudness. Perception \& Psychophysics, 3, 409-414. doi:10.3758/BF03205747

Hope, L., Gabbert, F., \& Fisher, R. P. (2011). From laboratory to the street: Capturing witness memory using the Self-Administered Interview. Legal and Criminological Psychology, 16, 211-226. doi:10.1111/j.20448333.2011.02015.x

Horry, R., Halford, P., Brewer, N, Milne, R., \& Bull, R. (2014). Archival analyses of eyewitness identification test outcomes: What can they tell us about eyewitness memory? Law and Human Behavior, 38, 94-108. doi:10.1037/lhb0000060

Houston, K. A., Hope, L., Memon, A., \& Read, J. D. (2013). Expert testimony on eyewitness evidence: In search of common sense. Behavioral Sciences and the Law, 31, 637-651. doi:10.1002/bsl.2080 
Horvath, M. A. H., \& Kelly, L. (2009). Multiple perpetrator rape: Naming an offence and initial research findings. Journal of Sexual Aggression, 15, 83-96. doi:10.1080/13552600802653818

Howarth, C. I., \& Bulmer, M. G. (1956). Non-random sequences in visual threshold experiments. Quarterly Journal of Experimental Psychology, 8, 163-171. doi:10.1080/17470215608416816

Hsu, S. M., \& Yang, L. X. (2013). Sequential effects in facial expression categorization. Emotion, 13, 573-586. doi:10.1037/a0027285

Innocence Project. (2017). http://www.innocenceproject.org/Last visited September 25, 2017.

Jarosz, A. F., \& Wiley, J. (2014). What are the odds? A practical guide to computing and reporting Bayes factors. The Journal of Problem Solving, 7, 2-9. doi:10.7771/1932-6246.1167

JASP Team (2017). JASP (Version 0.8.1.2)[Computer software].

Jenkins, R., White, D., Van Montfort, X., \& Burton, A. M. (2011). Variability in photos of the same face. Cognition, 121, 313-323. doi:10.1016/j.cognition.2011.08.001

Johnson, M. K., Hashtroudi, S., \& Lindsay, D. S. (1993). Source monitoring. Psychological Bulletin, 114, 3-28.

Jones, M., Love, B. C., \& Maddox, W. T. (2006). Recency effects as a window to generalization: Separating decisional and perceptual sequential effects in category learning. Journal of Experimental Psychology: Learning, Memory, and Cognition, 32, 316-332. doi:10.1.1.138.7280

Juodis, M., Woodworth, M., Porter, S., \& Ten Brinke, L. (2009). Partners in crime: A comparison of individual and multiple perpetrator homicides. Criminal Justice and Behavior, 36, 824-839. doi:10.1177/0093854809337822

Kan, I. P., Giovanello, K. S., Schnyer, D. M., Makris, N., \& Verfaellie, M. (2007). Role of the medial temporal lobes in relational memory: Neuropsychological evidence from a cued recognition paradigm. Neuropsychologia, 45, 2589-2597. doi:10.1016/j.neuropsychologia.2007.03.006

Kask, K., \& Bull, R. (2009). The effects of different presentation methods on multi-ethnicity face recognition. Psychology, Crime \& Law, 15, 73-89. doi:10.1080/10683160802131131

Kassin, S. M., Tubb, V. A., Hosch, H. M., \& Memon, A. (2001). On the" general acceptance" of eyewitness testimony research: A new survey of the experts. American Psychologist, 56, 405. doi:10.1037//0003066X.56.5.405

Kovera, M. B., \& Evelo, A. J. (2017). The case for double-blind lineup administration. Psychology, Public Policy, and Law, 23, 421. doi:10.1037/law0000139

Krix, A. C. (2015). Obtaining information from eyewitnesses: Effects of retrieval support in eyewitness interviews (Doctoral dissertation, Maastricht University).

Krix, A. C., Sauerland, M., Lorei, C., \& Rispens, I. (2015). Consistency across repeated eyewitness interviews: Contrasting police detectives' beliefs with actual eyewitness performance. PloS One, 10, e0118641. doi:10.1371/journal.pone.0118641

Leippe, M. R. (1995). The case for expert testimony about eyewitness memory. Psychology, Public Policy, and Law, 1, 909. doi:10.1037/1076-8971.1.4.909

Lewin, C., \& Herlitz, A. (2002). Sex differences in face recognition-Women's faces make the difference. Brain and Cognition, 50, 121-128. doi:10.1016/S0278-2626(02)00016-7

Lickel, B., Hamilton, D. L., Wieczorkowska, G., Lewis, A., Sherman, S. J., \& Uhles, A. N. (2000). Varieties of groups and the perception of group entitativity. Journal of Personality and Social Psychology, 78, 223-246. doi:10.1037//0022-3514.78.2.223

Liem, M., Ganpat, S., Granath, S., Hagstedt, J., Kivivuori, J., Lehti, M., \& Nieuwbeerta, P. (2013). Homicide in Finland, the Netherlands, and Sweden: First findings from the European Homicide Monitor. Homicide Studies, 17, 75-95. doi:10.1177/1088767912452130

Lilienfeld, S. O., Lynn, S. J., Ruscio, J., \& Beyerstein, B. L. (2011). 50 great myths of popular psychology: Shattering widespread misconceptions about human behavior. Chichester, UK: John Wiley \& Sons

Loftus, E. F. (2005). Planting misinformation in the human mind: A 30-year investigation of the malleability of memory. Learning \& Memory, 12, 361-366. doi:10.1101/Im.94705

Ma, D. S., Correll, J., \& Wittenbrink, B. (2015). The Chicago face database: A free stimulus set of faces and norming data. Behavior Research Methods, 47, 1122-1135. doi:10.3758/s13428-014-0532-5

Macmillan, N. A., \& Creelman, C. D. (2005). Detection theory: A user's guide (2nd ed.). Mahwah, NJ: Lawrence Erlbaum Associates

Malmberg, K. J., \& Annis, J. (2012). On the relationship between memory and perception: Sequential dependencies in recognition memory testing. Journal of Experimental Psychology: General, 141, 233-259. doi:10.1037/a0025277 
Malmberg, K. J., Lehman, M., Annis, J., Criss, A. H., \& Shiffrin, R. M. (2014). Consequences of testing memory. Psychology of Learning and Motivation, 61, 285-313. doi:10.1016/B978-0-12-8002834.00008-3

Malpass, R. S., \& Devine, P. G. (198I). Eyewitness identification: Lineup instructions and the absence of the offender. Journal of Applied Psychology, 66, 482- 489. doi:10.1037/0021-9010.66.4.482

Mandler, G. (1980). Recognizing: The judgment of previous occurrence. Psychological Review, 87, $252-271$. doi:10.1037/0033-295X.87.3.252

Mansour, J. K., Beaudry, J. L., \& Lindsay, R. C. L. (2017). Are multiple-trial experiments appropriate for eyewitness identification studies? Accuracy, choosing, and confidence across trials. Behavior Research Methods, $1-$ 20. doi:10.3758/s13428-017-0855-0

Martinez, A. M., \& Benavente, R. (1998). The AR face database (CVC Technical Report No. 24). Barcelona, Spain: Universitat Autonoma de Barcelona, Computer Vision Center.

Matthews, W. J., \& Stewart, N. (2009). The effect of inter-stimulus interval on sequential effects in absolute identification. The Quarterly Journal of Experimental Psychology, 62, 2014-2029. Doi:10.1080/17470210802649285

Megreya, A. M., \& Bindemann, M. (2012). Identification accuracy for single and double-perpetrator crimes: Does accomplice gender matter? British Journal of Psychology, 103, 439-453. doi:10.1111/j.20448295.2011.02084.x

Megreya, A. M., \& Burton, A. M. (2006). Recognising faces seen alone or with others: When two heads are worse than one. Applied Cognitive Psychology, 20, 957-972. doi:10.1002/acp.1243

Mickes, L., Flowe, H. D., \& Wixted, J. T. (2012). Receiver operating characteristic analysis of eyewitness memory: Comparing the diagnostic accuracy of simultaneous versus sequential lineups. Journal of Experimental Psychology: Applied, 18, 361-376. doi:10.1037/a0030609

Murdock Jr, B. B. (1962). The serial position effect of free recall. Journal of Experimental Psychology, 64, $482-488$. doi:10.1037/h0045106

Neuschatz, J. S., Deah, S. L., Fairless, A. H., Powers, R. A., Neuschatz, J. S., Goodsell, C. A., \& Toglia , M. P. (2007). The mitigating effects of suspicion on post-identification feedback and on retrospective eyewitness memory. Law and Human Behavior,31, 231-247. doi:10.1007/s10979-006-9047-7.

Nortje, A. Tredoux, C.G., \& Vredeveldt, A. (2017). How many faces can we remember? Why this matters when assessing eyewitnesses. In M. Bindemann \& A. Megreya (Eds.), Face processing: Systems, disorders and cultural disorders. New York: NOVA Science Publishers.

Palermo, R., \& Rhodes, G. (2002). The influence of divided attention on holistic face perception. Cognition, 82, 225257. doi:10.1016/S0010-0277(01)00160-3

Palmer, M. A., Brewer, N., \& Horry, R. (2013). Understanding gender bias in face recognition: Effects of divided attention at encoding. Acta Psychologica, 142, 362-369. doi:10.1016/j.actpsy.2013.01.009

Palmer, M. A., Brewer, N., \& Weber, N. (2010). Postidentification feedback affects subsequent eyewitness identification performance. Journal of Experimental Psychology: Applied, 16, 387-398. doi:10.1037/a0021034

Palmer, M. A., Brewer, N., \& Weber, N. (2012). The information gained from witnesses' responses to an initial "blank" lineup. Law and Human Behavior, 36, 439-447. doi:10.1037/h0093939

Palmer, M. A., Sauer, J. D., \& Holt, G. A. (2017). Undermining position effects in choices from arrays, with implications for police lineups. Journal of Experimental Psychology: Applied, 23, 71-84. doi:10.1037/xap0000109

Perfect, T. J., \& Harris, L. J. (2003). Adult age differences in unconscious transference: Source confusion or identity blending? Memory \& cognition, 31, 570-580.

Police and Criminal Evidence Act (PACE) (1984). Codes of Practice (Code D). (2013). http://police.homeoffice.gov.uk/operational-policing/powers-pace-codes/pace-codeintro/ (accessed 10 June, 2017).

Police Executive Research Forum (2013). A National Survey of Eyewitness Identification Procedures in Law Enforcement Agencies (Publication No. 242617). Washington D.C.: National Institute of Justice. Retrieved from: https://www.ncjrs.gov/pdffiles1/nij/grants/242617.pdf

Polyn, S. M., Norman, K. A., \& Kahana, M. J. (2009). A context maintenance and retrieval model of organizational processes in free recall. Psychological Review, 116, 129-156. doi:10.1037/a0014420

Preston, A. R., Shrager, Y., Dudukovic, N. M., \& Gabrieli, J. D. (2004). Hippocampal contribution to the novel use of relational information in declarative memory. Hippocampus, 14, 148-152. doi:10.1002/hipo.20009 
Rikspolisstyrelsen (2005). Vittneskonfrontation [Eyewitness identification procedures]. Accessed from: https://polisen.se/Global/www\%20och\%20Intrapolis/Rapporterutredningar/01\%20Polisen\%20nationellt/Ovriga\%20rapporterutredningar/Vittneskonfrontation_2005.pdf

Rotello, C. M., Macmillan, N. A., Hicks, J. L., \& Hautus, M. (2006). Interpreting the effects of response bias on remember-know judgments using signal-detection and threshold models. Memory \& Cognition, 34, 1598-1614. doi:10.3758/BF03195923

Sandholtz, N., Langton, L., \& Planty, M. (2013). Hate crime victimization, 2003-2011 (Special report NCJ, 241291). U.S. Department of Justice, Office of Justice Programs, Bureau of Justice Statistics.

Sauer, J. D., Brewer, N., \& Weber, N. (2008). Multiple confidence estimates as indices of eyewitness memory. Journal of Experimental Psychology: General, 137, 528-547. doi:10.1037/a0012712

Sauer, J. D., Brewer, N., Zweck, T., \& Weber, N. (2010). The effect of retention interval on the confidence-accuracy relationship for eyewitness identification. Law and Human Behavior, 34, 337-347. doi:10.1007/s10979-009-9192-x

Sauerland, M., Krix, A. C., \& Merckelbach, H. (2016). Konstruktion, Durchführung und Beurteilung von Gegenüberstellungen sind mehr als gesunder Menschenverstand [Lineup construction, administration and evaluation are more than just common sense]. Recht \& Psychiatrie, 34, 11-17.

Schacter, D. L. (1999). The seven sins of memory: Insights from psychology and cognitive neuroscience. American Psychologist, 54, 182-203. doi:10.1037/0003-066X.54.3.182

Schiff, W., Banka, L., \& de Bordes Galdi, G. (1986). Recognizing people seen in events via dynamic" mug shots". The American Journal of Psychology, 219-231. doi:10.2307/1422276

Schifferstein, H. N. J, \& Frijters, J. E. R. (1992). Contextual and sequential effects on judgments of sweetness intensity. Attention, Perception, \& Psychophysics, 52, 243-255. doi:10.3758/BF03209142

Schifferstein, H. N. J., \& Kuiper, W. E. (1997). Sequence effects in hedonic judgments of taste stimuli. Attention, Perception, \& Psychophysics, 59, 900-912. doi:10.3758/BF03205507

Shapiro, P. N., \& Penrod, S. (1986). Meta-analysis of facial identification studies. Psychological Bulletin, 100, 139156. doi:10.1037/0033-2909.100.2.139

Shepherd, J.W. (1983). Identification after long delays. In S. M. A. Lloyd-Bostock, \& B. R. Clifford (Eds.), Evaluating witness evidence (pp. 173-187). Chichester: Wiley.

Simmons, J. P., Nelson, L. D., \& Simonsohn, U. (2011). False-positive psychology: Undisclosed flexibility in data collection and analysis allows presenting anything as significant. Psychological Science, 22, 1359-1366. doi:10.1177/0956797611417632

Smith, S. M., \& Vela, E. (2001). Environmental context-dependent memory: A review and metaanalysis. Psychonomic Bulletin \& Review, 8, 203-220. doi:10.3758/BF03196157

State v. Coley, 32 S.W.3d 831 (Tenn. 2000).

Statistics Canada (2016). Table 253-0008 - Homicide survey, gang-related homicide, by region, annual, CANSIM (database). (accessed: September 26, 2017)

Steblay, N. M. (1997). Social influence in eyewitness recall: A meta-analytic review of lineup instruction effects. Law and Human Behavior, 21, 283-297. doi:10.1023/A:1024890732059

Steblay, N. K., Dysart, J. E., \& Wells, G. L. (2011). Seventy-two tests of the sequential lineup superiority effect: A meta-analysis and policy discussion. Psychology, Public Policy, and Law, 17, 99-139. doi:10.1037/a0021650

Steblay, N. K., Wells, G. L., \& Douglass, A. B. (2014). The eyewitness post identification feedback effect 15 years later: Theoretical and policy implications. Psychology, Public Policy, and Law, 20, 1. doi: 10.1037/law0000001

Stewart, N., Brown, G. D., \& Chater, N. (2005). Absolute identification by relative judgment. Psychological Review, 112, 881-911. doi:10.1037/0033-295X.112.4.881

Taubert, J., Van der Burg, E., \& Alais, D. (2016). Love at second sight: Sequential dependence of facial attractiveness in an on-line dating paradigm. Scientific Reports, 6, 22740. doi:10.1038/srep22740

The output for this paper was generated using Qualtrics software. Copyright (C2016 Qualtrics. Qualtrics and all other Qualtrics product or service names are registered trademarks or trademarks of Qualtrics, Provo, UT, USA. http://www.qualtrics.com

The psychological image collection at Stirling (PICS) (n.d.). University of Stirling Psychology Department. Retrieved from http://pics.psych.stir.ac.uk/.

Thomson, D. M., \& Tulving, E. (1970). Associative encoding and retrieval: Weak and strong cues. Journal of Experimental Psychology, 86, 255-262. doi:10.1037/h0029997 
Treisman, M., \& Williams, T. C. (1984). A theory of criterion setting with an application to sequential dependencies. Psychological Review, 91, 68-111. doi:10.1037/0033-295X.91.1.68

Tulving, E., \& Thomson, D. M. (1973). Encoding specificity and retrieval processes in episodic memory. Psychological review, 80, 352-373. doi:10.1037/h0020071

Tupper, N., Sauerland, M., Sauer, J., \& Hope, L. (2018). Eyewitness identification procedures for multiple perpetrator crimes: A survey of police in Sweden, Belgium, and the Netherlands. Manuscript in preparation.

United States v. Libby, 461 F. Supp. 2d.12 (D.D.C. 2006).

Van Zandt, T. (2000). ROC curves and confidence judgments in recognition memory. Journal of Experimental Psychology: Learning, Memory, and Cognition, 26, 582-600.

Vokey, J. R., \& Read, J. D. (1992). Familiarity, memorability, and the effect of typicality on the recognition of faces. Memory \& Cognition, 20, 291-302. doi:10.3758/BF03199666

Vulkan, N. (2000). An economist's perspective on probability matching. Journal of Economic Surveys, 14, $101-118$. doi:10.1111/1467-6419.00106

Wagenmakers, E. J., Love, J., Marsman, M., Jamil, T., Ly, A., Verhagen, J., \& Meerhoff, F. (2017). Bayesian inference for psychology. Part II: Example applications with JASP. Psychonomic Bulletin \& Review, 25, 58-76. doi:10.3758/s13423-017-1323-7

Watkins, M. J., Ho, E., \& Tulving, E. (1976). Context effects in recognition memory for faces. Journal of Verbal Learning and Verbal Behavior, 15, 505-517. doi:10.1016/0022-5371(76)90045-1

Weber, N., \& Brewer, N. (2004). Confidence-accuracy calibration in absolute and relative face recognition judgements. Journal of Experimental Psychology: Applied, 10, 156-172. doi:10.1037/1076898X.10.3.156

Wells, G. L., \& Bradfield, A. L. (1998). "Good, you identified the suspect": Feedback to eyewitnesses distorts their reports of the witnessing experience. Journal of Applied Psychology, 83, 360-376. doi:10.1037/00219010.83.3.360

Wells, G. L., \& Luus, E. (1990). Police lineups as experiments: Social methodology as a framework for properlyconducted lineups. Personality and Social Psychology Bulletin, 16, 106-117.

Wells, G. L., Malpass, R. S., Lindsay, R. C. L., Fisher, R. P., Turtle, J. W., \& Fulero, S. M. (2000). From the lab to the police station: A successful application of eyewitness research. American Psychologist, 55, 581-598.

Wells, G. L., Memon, A., \& Penrod, S. D. (2006). Eyewitness evidence: Improving its probative value. Psychological Science in the Public Interest, 7, 45-75. doi:10.1111/j.1529-1006.2006.00027.x

Wells, E. C., \& Pozzulo, J. D. (2006). Accuracy of eyewitnesses with a two-culprit crime: Testing a new identification procedure. Psychology, Crime and Law, 12, 417-427. doi:10.1080/10683160500050666

Wickham, L. H., Morris, P. E., \& Fritz, C. O. (2000). Facial distinctiveness: Its measurement, distribution and influence on immediate and delayed recognition. British Journal of Psychology, 91, 99-123. doi:10.1348/000712600161709

Wilcock, R., Crane, L., Hobson, Z., Nash, G., Kirke-Smith, M., \& Henry, L. (2018). Supporting child witnesses during identification lineups: Exploring the effectiveness of Registered Intermediaries. Applied Cognitive Psychology. doi:10.1002/acp.3412

Winograd, E., \& Rivers-Bulkeley, N. T. (1977). Effects of changing context on remembering faces. Journal of Experimental Psychology: Human Learning and Memory, 3, 397-405. doi:10.1037/0278-7393.3.4.397

Wise, R. A., Pawlenko, N. B., Safer, M. A., \& Meyer, D. (2009). What US prosecutors and defence attorneys know and believe about eyewitness testimony. Applied Cognitive Psychology, 23, 1266-1281. doi: 10.1002/acp.1530

Wright, D. B., \& Sladden, B. (2003). An own gender bias and the importance of hair in face recognition. Acta Psychologica, 114, 101-114. doi:10.1016/S0001-6918(03)00052-0

Zhou, B., Lapedriza, A., Xiao, J., Torralba, A., \& Oliva, A. (2014). Learning deep features for scene recognition using places database. Advances in Neural Information Processing Systems, 487-495. 
REFERENCES 


\section{SUMMARY}

The over-arching aim of the research conducted for this thesis was to examine underlying issues in memory and decision-making that impact eyewitness identification procedures in the context of multiple perpetrator crimes. In one survey and five experiments, we (i) explored key concerns in multiple perpetrator identifications in police practice in three EU countries (Police Survey), (ii) tested the independence of multiple identification decisions made successively (Experiments 1, 2 and 3) and (iii) examined the purported utility of using other faces as contextual cues for recognizing the faces of multiple perpetrators (Experiments 4 and 5).

In the survey we asked police officers (from Sweden, Belgium, and the Netherlands) to describe how agencies in various countries conduct and regulate identification procedures with multiple perpetrators. Results demonstrated that practice converges when it comes to the use of sequential, photographic lineups, but diverges between and within countries on issues such as whether or not suspects of multiple perpetrator crimes should be placed in separate lineups. Results specifically highlight the role of context as one critical area for future research in identification for multiple perpetrator crimes (i.e., placing multiple suspects in the same lineup or asking eyewitnesses to look for a specific suspect).

In Experiments 1 and 2, participants watched a mock crime film involving three perpetrators and later made three showup identification decisions, one showup for each perpetrator. Experiments 1 and 2 used similar procedures, with the exception of varied patterns of target-presence. Across both experiments, evidence for sequential dependencies for choosing behavior was inconsistent. In Experiment 1, responses on the second, target-present showup assimilated towards previous choosing. However, in Experiment 2, responses on the second showup contrasted previous choosing regardless of target-presence. Experiment 3 examined whether methodological differences between the recognition and eyewitness paradigms used in previous research on sequential dependencies might account for the inconsistent findings in Experiments 1 and 2 . Participants studied pairs of words, landscapes, or faces, and were later tested for recognition. Sequential dependencies were detected in recognition decisions over many trials, including recognition for faces: the probability of a yes response on the current trial increased if the previous response was also yes (vs. no). However, choosing behavior on previous trials did not predict individual recognition decisions on the current trial. This suggests that the integrity of identification and recognition decisions is not likely to be 
impacted by making the multiple decisions in a row, and that it is more important to focus on initial bias in choosing behavior that is maintained throughout the trials.

In Experiments $\mathbf{4}$ and 5, we sought to (i) replicate facilitative effects in cued face recognition, to (ii) investigate the mechanisms underlying those effects, and (iii) determine whether such effects would extend to more than two faces. Participants encoded sets of individual, paired, or groups of four faces and were tested with no cues, correct cues (a face previously studied with the target test face), or incorrect cues (a never-before-seen face). Hit rates were not affected by either cue type or face encoding condition, but cuing of any kind (correct or incorrect) appeared to provide a protective buffer against false alarms (i.e., false recognition) in the two- and four-face conditions through reduced response bias. Our findings suggest that cued face recognition may be a useful technique to use for reducing false recognition rates in contexts with multiple faces. Throughout the thesis, we argue for the systematic examination of influential factors that are both unique and inherent to practice, memory, and decision-making for multiple perpetrator identification and recognition. 
SUMMARY 


\section{VALORIZATION}

\section{RELEVANCE}

Eyewitness research, and by extension this thesis, is relevant because eyewitness evidence remains an important source of evidence in the legal system. Indeed, thousands of identification lineups and showups are conducted around the world each year, and are used for the investigation and prosecution of suspected perpetrators. Because incidents involving multiple perpetrators make up a substantial proportion of all crime, many of these lineups and showups are conducted in cases of multiple perpetrator crimes. However, guidance for procedures is currently lacking and the procedures that do exist are not evidence-based. This is not surprising given that the research needed to provide guidance for evidence-based procedures is itself lacking. This thesis is relevant because it begins to fill the gap in our knowledge on the recognition of multiple faces and the decisions to identify those faces or not; Thus it is the first step towards crafting evidence-based identification procedures in cases with multiple perpetrators.

\section{TARGET POPULATION AND PRODUCTS}

In using science to shape public policy, it is crucial to realistically judge the state of our knowledge so that we can respect the limits of our capacity to make evidence-based recommendations. In this vein, this thesis is not an end-product; this thesis is a beginning of investigation on multiple perpetrator identification that will eventually be integrated into the general scientific consensus. This consensus can eventually be used by experts to teach juries, lawyers, and police about memory for multiple perpetrator crimes, by lawyers to defend their clients, and by judges to regulate appropriate evidence to be submitted to the court and to evaluate its probative value. It can also be used to shape police protocols that are specifically designed to support eyewitness memory and prevent the contamination of eyewitness identification decisions in multiple perpetrator crimes. This in turn enhances the probability of obtaining valuable eyewitness identifications in this context. Such protocols could be used in police training and police manuals in those states or countries that are amenable to adopting evidence-based practice. All of these uses serve the ever-present goal in eyewitness research: to increase the number of perpetrators that are rightfully convicted for their crimes, to reduce the number of innocent suspects serving time for a crime they did not commit, and thus to prevent miscarriages of justice. 


\section{INNOVATION}

The aim of this thesis was to examine memory and eyewitness identification decisions in cases where there were multiple perpetrators. The possibility of multiple perpetrators being present at a crime, or the possible consequences of that presence, is oftforgotten or perhaps oft-ignored within the otherwise prolific research field of eyewitness identification. To date, only a few studies have addressed the topic of eyewitness identifications of multiple perpetrator crimes, and this thesis seeks to contribute to that knowledge.

Chapter 2 presents an initial picture of the state of police practice for constructing, administering, recording, and evaluating eyewitness identification decisions when multiple suspects are involved. In fact, this is the second of only two surveys that exist on the issue. The current survey is also unique in that it was the first to do so outside of the U.K. This international perspective on police practice allows researchers to examine current protocols, test new solutions, and hopefully improve police and eyewitness experience with multiple perpetrator identifications. Officers reported a lack of regulations regarding multiple perpetrator identification, and thus generally intuitive decisions on withhold the context for the eyewitness identification (i.e., this lineup is for the man you said was holding the gun) and whether or not to place suspects in separate or the same lineups. They also emphasized difficulties for eyewitnesses to distinguish between multiple perpetrators.

Chapters 3 and 4 investigate factors that are both unique and inherent to multiple perpetrator crimes - factors that we can and should consider when adapting identification protocols. In particular, Chapter 3 presents the first known experiments testing for relationships between multiple, ostensibly-independent identification decisions related to the different perpetrators of a multiple perpetrator crime. Within eyewitness identification research on single perpetrator crimes, it is widely accepted that suspects should always be placed in separate lineups in order to decrease the probability of a misidentification. These lineups are inevitably related because the multiple suspects always refer to the same perpetrator. In the case of a multiple perpetrator crime, multiple suspects may be related to different perpetrators, and identification decisions for the suspect(s) regarding one perpetrator should not impact the identification decision(s) for another. This independence between decisions is important, for example, for prosecuting the different co-perpetrators of the crime. That these decisions can be independent from each other has implications for recommendations for police protocols. For example, we can ask whether an eyewitness should be allowed to make multiple identification decisions on the same day.

Another decision on lineup administration include the golden rule of lineup construction: that suspects should always be placed in separate lineups. This rule implicitly refers to the suspects of a single perpetrator crime. In the case of multiple perpetrator 
crimes, police manuals (e.g., the U.K. and the Netherlands, see Chapter 2) are allowing multiple suspects to be placed in the same lineup under certain conditions (i.e., when presented in photographic lineups vs. live lineups). It is important for us to understand if this is an appropriate decision and, if so, under what conditions. Thus, Chapter 4 presents experiments examining the possibility of a memory advantage when intentionally violating the independence between multiple recognition decisions. These experiments include new developments in methodology and statistics. They are innovative in that they include groups of more than two faces in the encoding and recognition phases. We found that recognition decisions using other faces as cues reduced the rate of false recognition and may be a useful tool in the future for identification procedures.

\section{IMPLEMENTATION AND KNOWLEDGE DISSEMINATION}

The articles comprising this thesis have all been presented at international conferences on psychology and law or applied cognition and are under review to be published in academic journals. Publication is not only important to communicate new psychological findings to researchers who may conduct experiments related to multiple perpetrator identification or multiple face recognition, but also to remind researchers to be cognizant of the importance of multiple vs. single perpetrator distinctions in their own research. For example, it would be useful to the field of multiple perpetrator identification if researchers conducting police surveys include questions about multiple perpetrator crimes; it would be useful if researchers conducting archival studies documented multiple perpetrator crimes with details on police decisions; lastly, it would be useful if researchers using multiple perpetrators in their stimulus videos consistently test for relationships between the identification decisions. The main findings of this dissertation and the ideas for future research have also been mentioned in lectures to an international body of students, who may be future researchers, lawyers, police, judges, or even jury members around the world. 


\section{ACKNOWLEDGMENTS}

I would like to acknowledge and thank the European Union and the Erasmus Mundus Joint Doctorate Program in Legal Psychology for funding this PhD fellowship. I would particularly like to extend my deepest gratitude to the House of Legal Psychology for developing this incredible international collaboration with its vast and varied network of candidates and established researchers from all over the world. Thank you to all of the supervisors, mentors, and visitors to the program for contributing their time and resources to our training, for imparting their knowledge, and for offering their shoulders on which our dwarfly selves can stand.

In that vein, I would especially like to thank my direct supervisors: Melanie Sauerland, Lorraine Hope, Jim Sauer for their mentorship these last four years. In particular for consistently pulling me (and my writing) out of the clouds and down to the ground, for your infectious passion for science, and for providing support, critiques, and advice throughout.

Thank you to all of my colleagues, office-mates, and friends in Portsmouth and Maastricht for the many stats-chats, psych-chats life-chats, and soundboard sessions; Andy and Joanne for your constant companionship and invaluable perspective through all the various locations of this nomadic, academic life; Nathalie and Lili for your enthusiastic cheerleading, celebrating, and commiserating as the situation required, for your Germanly enforcement of work-life balance, and for much-needed stability; Irena and Zi for your buoying reassurance when I needed it, and your tough love when I needed that, too. Thank you to the other candidates in the House for making this experience so lively and remarkable.

Thank you to Ultimaas for being my ever-evolving family away from home. Tiffany, thank you for pulling me away from the books and out into the fresh air, for pulling me out of Maastricht and over to the many exotic locations of other sports fields, and for so many entertaining, absurd, and priceless moments at the end of long days and long weeks.

Lastly, I would like to thank my brothers, Kristofer and Jackson, and my parents for their constant support - each in their own way. It is no easy job, but I am especially grateful for your patience and encouragement from across the ocean time and time again. My parents instilled in me a love for learning and the value of follow-through, both of which have brought me to where I am today. 


\section{CURRICULUM VITAE}

Nina Tupper was born on June 12, 1991 in Providence, Rhode Island (U.S.). In 2009 she graduated from Kennebunk High School, after which she moved to Guatemala to study Spanish and work for the NGO Safe Passage (Camino Seguro). In 2010, she started a liberal arts degree at Mount Holyoke College in South Hadley, Massachusetts to pursue a degree in Romance Languages and entered her first psychology course. In 2011, she transferred to Bates College in Lewiston, Maine, where she studied Psychology with a focus in Social. She graduated in May 2014 with a Bachelor of Arts, having completed and presented her thesis in eyewitness identification and the Post Identification Feedback Effect. In September 2014, she started her PhD Project with the Erasmus Mundus Joint Doctorate Program in Legal Psychology. Her project on eyewitness identification and recognition for multiple perpetrators was supervised by Prof. Dr. H.L.G.J. Merckelback and Dr. M. Sauerland in Maastricht, the Netherlands, by Prof. Dr. Lorraine Hope in Portsmouth, U.K., and by Dr. James Sauer in Tasmania, Australia. During the course of her PhD project, she spent 6 months at the University of Portsmouth with the Hope Applied Cognition (HAC) Lab. 


\section{PUBLICATIONS}

Tupper, N., Sauerland, M., Hope, L., \& Merckelbach, H.M.J. (2015). Seeing and believing: Common courtroom myths in eyewitness memory. In Mind, 28, 1-6. (see: http://www.inmind.org/article/seeing-and-believing-common-courtroom-myths-in-eyewitness-memory)

\section{PUBLICATIONS UNDER REVIEW}

Satchell, L., Davis, J.P., Julle-Daniere, E., Tupper, N., \& Marhsman, P., (2018) Superrecognising kernels of truth? Exploring the relationship between personality, person judgment accuracy and face recognition. Manuscript under review.

Tupper, N., Sauerland, M., Sauer, J., Broers, N., Charman, S., \& Hope, L. (2018). Eyewitness identification for multi-perpetrator crimes: Testing for sequential effects in multiple showup decisions. Revision invited.

Tupper, N., Sauer, J., Sauerland, M., Fu, I., \& Hope, L. (2018). Face value: Testing the utility of contextual face cues for face recognition. Manuscript accepted pending minor revisions.

Tupper, N., Sauerland, M., Sauer, J., \& Hope, L. (2018) Eyewitness identification procedures for multiple perpetrator crimes: A survey of police in Sweden, Belgium, and the Netherlands. Manuscript under revision.

\section{CONFERENCE PRESENTATIONS}

Tupper, N., Sauerland, M., Hope, L., Sauer, J., \& Charman, S. (2015). Identification for multiple perpetrator crimes: Testing the independence of multiple lineup decisions. Poster presented at the annual meeting of the European Association for Psychology and Law Conference (EAPL), Nuremberg, Germany.

Tupper, N., Sauerland, M., Hope, L., Sauer, J., Broers, N., Charman, S., \& Merckelbach, H. (2016). Eyewitness identification for multiple perpetrator crime: Testing for sequential effects in multiple identification decisions. Presentation at the annual meeting of the American Psychology and Law Society Conference (AP-LS), Atlanta, Georgia, USA.

Tupper, N., Sauerland, M., Sauer, J., Broers, N., Charman, S., \& Hope, L. (2016). Eyewitness identification for multi-perpetrator crimes: Testing for sequential effects in multiple showup 
decisions. Presentation at the biennial meeting of the Society for Applied Research in Memory and Cognition (SARMAC) Sydney, Australia.

Tupper, N., Sauer, J., Sauerland, M., Fu, I., \& Hope, L. (2016). Cued face recognition: Can we stretch the effect? Presentation at for the biennial meeting of SARMAC, Sydney, Australia.

Tupper, N., Sauerland, M., Sauer, J., \& Hope, L. (2018) Eyewitness identification procedures for multiple perpetrator crimes: A survey of police in Sweden, Belgium, and the Netherlands. Presentation at the annual meeting of the AP-LS, Memphis, Tennessee, USA.

\section{GRANTS, HONORS, AND SCHOLARSHIPS}

Awarded APA Div. 41 AP-LS Grants-in-Aid, 2016

Awarded AP-LS grant to attend Continuing Education Workshop: Testifying and Consulting as an Expert in Eyewitness Identification Cases with Karen Newirth, JD and Barry Scheck, JD, The Innocence Project, 2016

Best poster award- EAPL conference, 2015 
Cover design by Jackson Tupper 


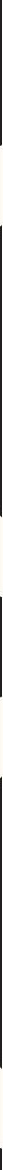

